CRYSTALLOGRAPHIC COMMUNICATIONS

ISSN 2056-9890

\section{Reduced 3,4'-bipyrazoles carrying thiophene and thiazole substituents: structures of two intermediates and two products}

\author{
Chayanna Harish Chinthal, ${ }^{\mathrm{a}}$ Hemmige S. Yathirajan, ${ }^{\mathrm{a} *}$ Nagaraja Manju, \\ Balakrishna Kalluraya, ${ }^{b}$ Sabine Foro ${ }^{c}$ and Christopher Glidewell ${ }^{d}$
}

Received 5 February 2021

Accepted 28 February 2021

Edited by D. Chopra, Indian Institute of Science Education and Research Bhopal, India

Keywords: heterocyclic compounds; reduced bipyrazoles; synthesis; crystal structure; regiochemistry; hydrogen bonding; supramolecular assembly.

CCDC references: 2065478; 2065477; 2065476; 2065475

Supporting information: this article has supporting information at journals.iucr.org/e

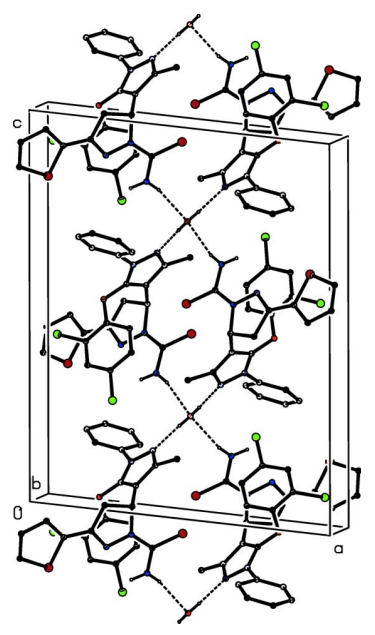

\section{Chemical context}

Heterocyclic compounds containing the pyrazole unit have been found to exhibit a wide range of biological activities, including antibacterial and antifungal activity (Rai et al., 2008; Isloor et al., 2009; Vijesh et al., 2013) and analgesic and antiinflammatory activity (Girisha et al., 2010; Isloor et al., 2010; Vijesh et al., 2013). It has also been found that the incorporation of a thiazole or thiazolone substituent often leads to enhanced activity (Sulthana et al., 2015; Havrylyuk et al., 2016), as does the incorporation of a thiophene substituent (Rostom et al., 2009; Bondock et al., 2010). In this connection, a procedure has recently been developed (Manju et al., 2019) for the synthesis of reduced 3,4'-bipyrazoles incorporating 
other heterocyclic units such as thiazole, thiazoline and thiophene as integral components. In brief, condensation of a 5-aryloxy-3-methyl-1-phenyl- $1 H$-pyrazole-4-carbaldehyde with 2-acetylthiophene gives the corresponding chalcone (Shaibah et al., 2020); chalcones of this type can undergo cyclocondensation reactions with semicabazide to provide the intermediate carbothioamides of type (I) (see Scheme). Further condensation of type (I) intermediates with diethyl acetylenedicarboxylate or with 4-bromophenacyl bromide gave the oxothiazolylidene ester (II) or the thiazole (III), respectively (see Scheme). Although the NMR spectra of the intermediates (I) and the products (II) and (III) contained all of the expected signals, it was not possible to establish uniquely from these data the regiochemistry of the cycloaddition reactions leading to their formation, and accordingly we have determined the structures of two representative intermediates (Ia) and (Ib) (Figs. 1 and 2) and of two representative products (II) (Fig. 3) and (III) (Fig. 4).
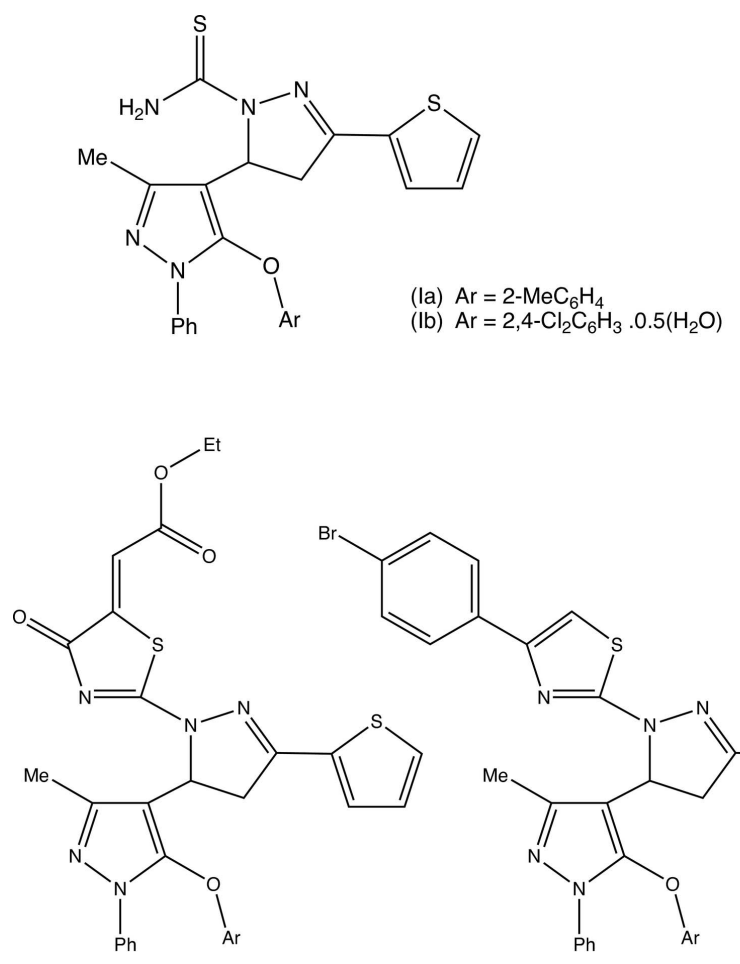

(II) $\mathrm{Ar}=2-\mathrm{MeC}_{6} \mathrm{H}_{4}$

(III) $\mathrm{Ar}=2,4-\mathrm{Cl}_{2} \mathrm{C}_{6} \mathrm{H}_{3}$

\section{Structural commentary}

Although compounds (Ia), (Ib), (II) and (III) were all crystallized under identical conditions, compound (Ib) crystallized as a hemihydrate, in which the water molecules lies across a twofold rotation axis, while the other three compounds all crystallized in solvent-free form. In each compound, the thiophene substituent is disordered over two sets of atomic sites (Section 6), whose relationship approximately corresponds to a rotation of $180^{\circ}$ about the bond C45-C452 (Figs.

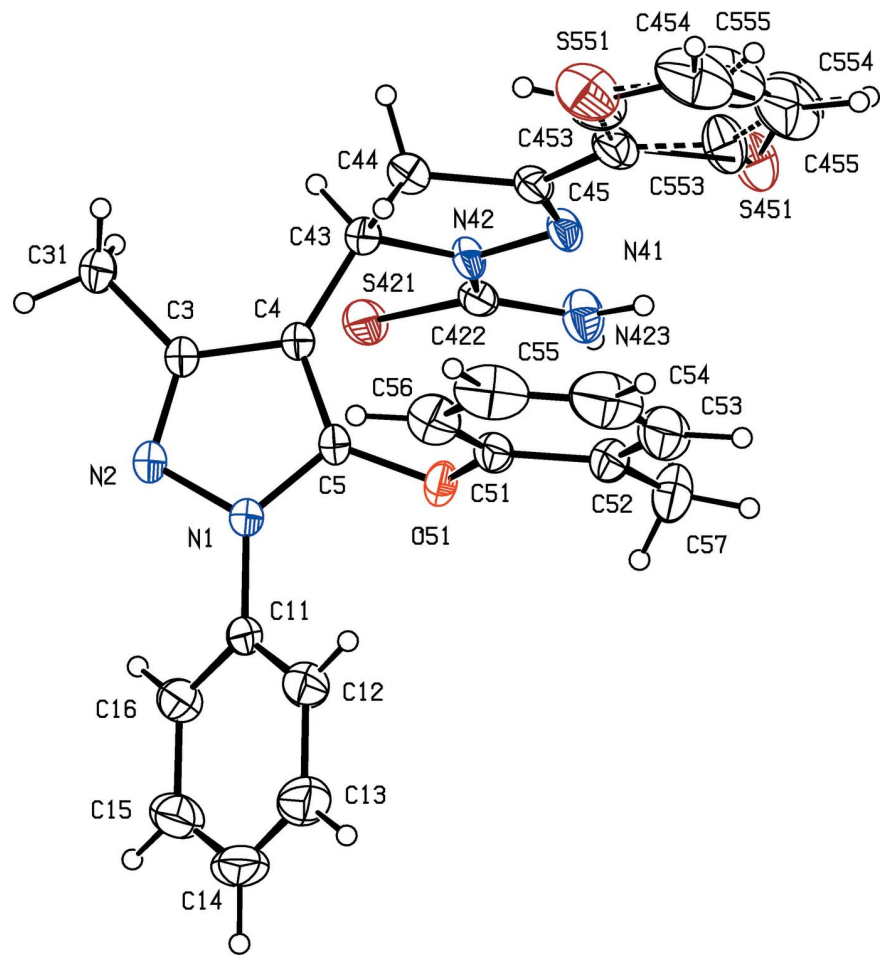

Figure 1

The molecular structure of compound (Ia) showing the atom-labelling scheme and the disorder in the thiophene unit, where the major disorder component is drawn using full lines and the minor disorder component is drawn using broken lines. Displacement ellipsoids are drawn at the $30 \%$ probability level.

1-4). That the cyclocondensation reactions between the chalcone precursors and thiosemicarbazide lead to the formation of new pyrazole rings indicates that it is the two $\mathrm{N}$

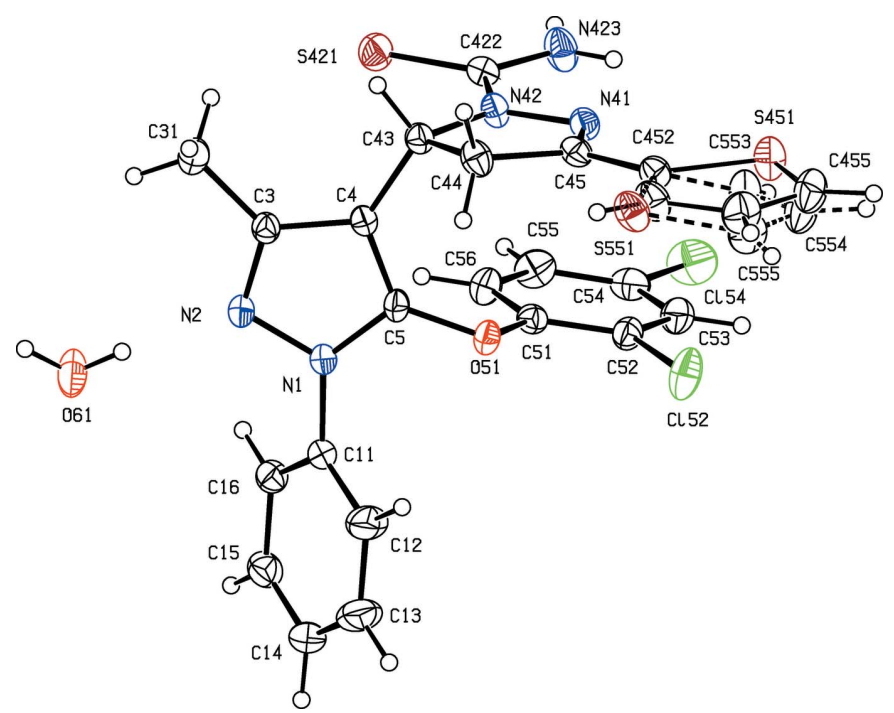

Figure 2

The structure of the independent components in compound (Ib) showing the atom-labelling scheme and the disorder in the thiophene unit, where the major disorder component is drawn using full lines and the minor disorder component is drawn using broken lines. The water molecule lies across a twofold rotation axis and the displacement ellipsoids are drawn at the $30 \%$ probability level. 


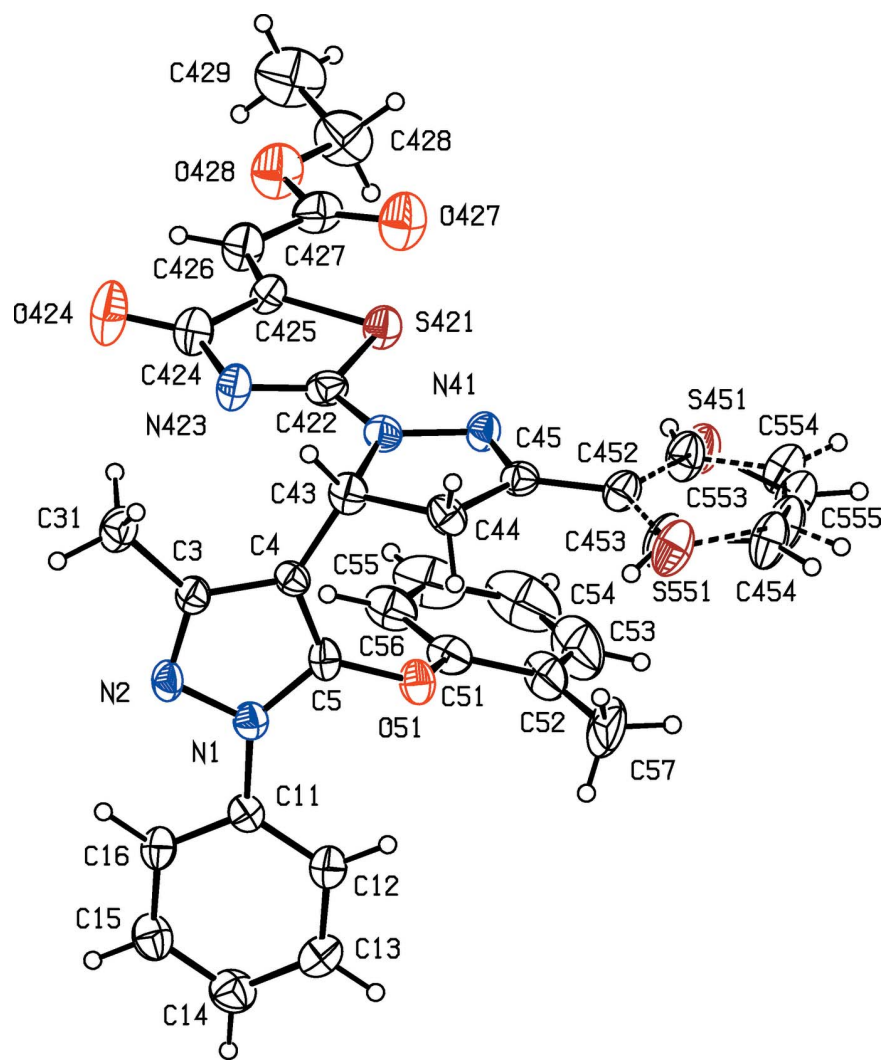

Figure 3

The molecular structure of compound (II) showing the atom-labelling scheme and the disorder in the thiophene unit, where the major disorder component is drawn using full lines and the minor disorder component is drawn using broken lines. Displacement ellipsoids are drawn at the $30 \%$ probability level.

atoms of the hydrazine unit in thiosemicarbazide that participate in this reaction step. If the participants had been the two $\mathrm{N}$ atoms either side of the thiocarbonyl unit, then the products

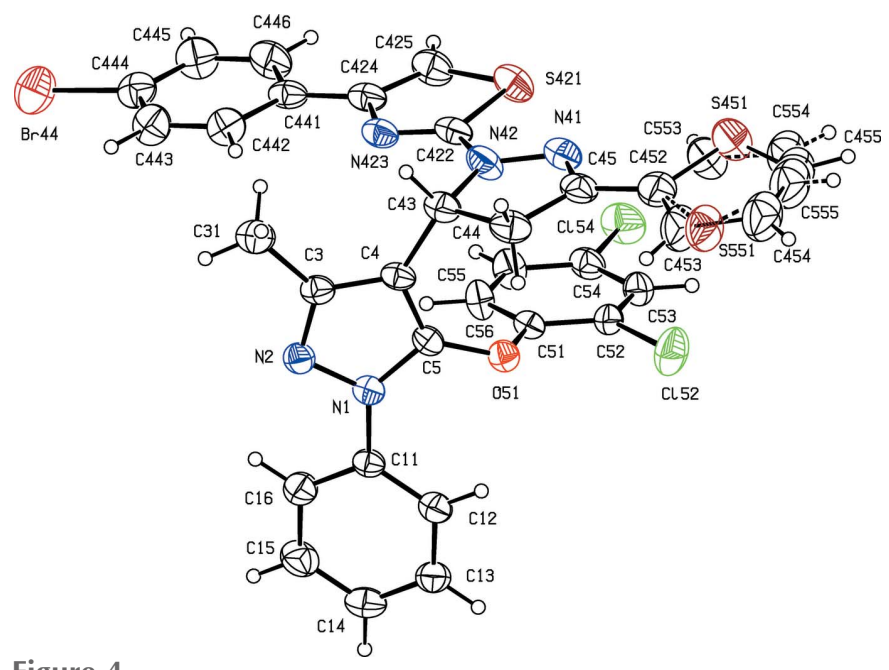

Figure 4

The molecular structure of compound (III) showing the atom-labelling scheme and the disorder in the thiophene unit, where the major disorder component is drawn using full lines and the minor disorder component is drawn using broken lines. Displacement ellipsoids are drawn at the $30 \%$ probability level.<smiles>Cc1nn(-c2ccccc2)c(O[AlH2])c1C1CC(c2cccs2)=NC(=S)N1N</smiles>

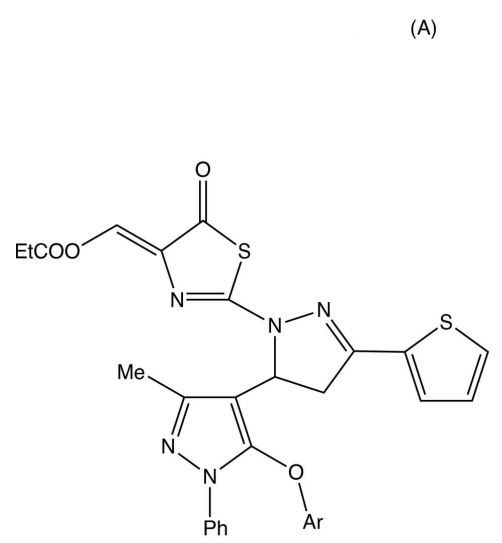

(B)

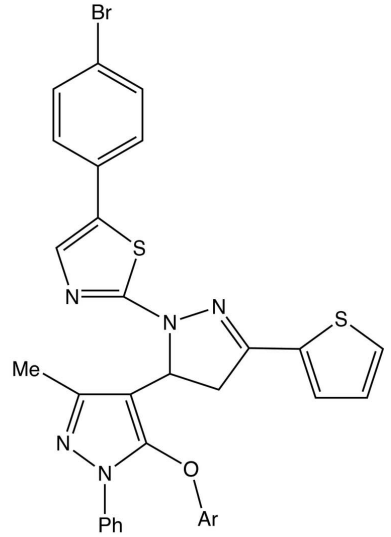

(C)
Figure 5

Possible regioisomers (A)-(C) of compounds (I)-(III), respectively.

would have been the regioisomers of type (A), containing a newly formed reduced pyrimidine ring in place of the pyrazole ring actually observed (Fig. 5). Similarly, in the cyclocondensation reactions between the carbothioamides (I) and either diethyl acetylenedicarboxylate or 4-bromophenacyl bromide to form (II) and (III), respectively, alternative regiochemistry is possible in each case, to yield products of types (B) and (C), respectively (Fig. 5). The X-ray analyses reported here have confirmed that the single products formed in each of these cyclocondensation reactions (Manju et al., 2019) have structures of types (I)-(III), as opposed to the possible alternative isomers $(\mathrm{A})-(\mathrm{C})$.

\section{Supramolecular features}

The supramolecular assembly of compound (Ia) is extremely simple: a single $\mathrm{N}-\mathrm{H} \cdots \mathrm{N}$ hydrogen bond (Table 1) links molecules that are related by translation into a $C(8)$ (Etter, 1990; Etter et al., 1990; Bernstein et al., 1995) chain running parallel to the [100] direction (Fig. 6), but there are no direction-specific interactions between adjacent chains.

Compound ( $\mathrm{Ib})$ is a hemihydrate in which the water component lies across a twofold rotation axis, and the supramolecular aggregation is more complex than that in (Ia). There is an $\mathrm{O}-\mathrm{H} \cdots \mathrm{N}$ hydrogen bond within the selected asymmetric unit (Table 1), and pairs of inversion-related bimolecular units of this type are linked by paired $\mathrm{N}-\mathrm{H} \cdots \mathrm{O}$ hydrogen bonds to form an $R_{4}^{4}(20)$ ring. Propagation of this motif by the action of the twofold rotation axes generates a chain of spiro-fused $R_{4}^{4}(20)$ rings running parallel to the [001] 
Table 1

Hydrogen bonds and short inter- and intramolecular contacts $\left(\AA{ }^{\circ}{ }^{\circ}\right)$.

$C g 1$ represents the centroid of the $\mathrm{C} 11-\mathrm{C} 16$ ring.

\begin{tabular}{|c|c|c|c|c|c|}
\hline Compound & $D-\mathrm{H} \cdots A$ & $D-\mathrm{H}$ & $\mathrm{H} \cdots A$ & $D \cdots A$ & $D-\mathrm{H} \cdots A$ \\
\hline \multirow[t]{2}{*}{ (Ia) } & $\mathrm{N} 423-\mathrm{H} 42 A \cdots \mathrm{N} 2^{\mathrm{i}}$ & $0.92(3)$ & $2.28(3)$ & 3.111 (3) & $150(2)$ \\
\hline & $\mathrm{N} 423-\mathrm{H} 42 A \cdots \mathrm{N} 41$ & $0.92(3)$ & $2.27(3)$ & $2.628(4)$ & $103(2)$ \\
\hline \multirow[t]{3}{*}{ (Ib) } & $\mathrm{O} 61-\mathrm{H} 61 \cdots \mathrm{N} 2$ & $0.88(3)$ & $2.03(3)$ & $2.900(2)$ & $176(2)$ \\
\hline & $\mathrm{N} 423-\mathrm{H} 42 A \cdots \mathrm{O} 61^{\mathrm{ii}}$ & $0.84(3)$ & $2.33(3)$ & $3.154(3)$ & $167(3)$ \\
\hline & $\mathrm{N} 423-\mathrm{H} 42 B \cdots \mathrm{N} 41$ & $0.85(3)$ & $2.27(3)$ & $2.648(3)$ & $107(2)$ \\
\hline \multirow[t]{3}{*}{ (II) } & $\mathrm{C} 13-\mathrm{H} 13 \cdots \mathrm{O} 424^{\mathrm{iii}}$ & 0.93 & 2.49 & $3.200(7)$ & 133 \\
\hline & $\mathrm{C} 54-\mathrm{H} 54 \cdots C g 1^{\mathrm{iv}}$ & 0.93 & 2.91 & $3.714(12)$ & 146 \\
\hline & $\mathrm{C} 553-\mathrm{H} 553 \cdots C g 1^{\mathrm{v}}$ & 0.93 & 2.92 & $3.76(5)$ & 151 \\
\hline
\end{tabular}

Symmetry codes: (i) $1+x, y, z$; (ii) $1-x, 1-y, 1-z$; (iii) $x,-1+y, z$; (iv) $2-x,-y, 1-z$; (v) $-1+x, y, z$.

direction, in which the centrosymmetric rings are centred at $(0.5,0.5,0.5 n)$ where $n$ represents an integer (Fig. 7). Within

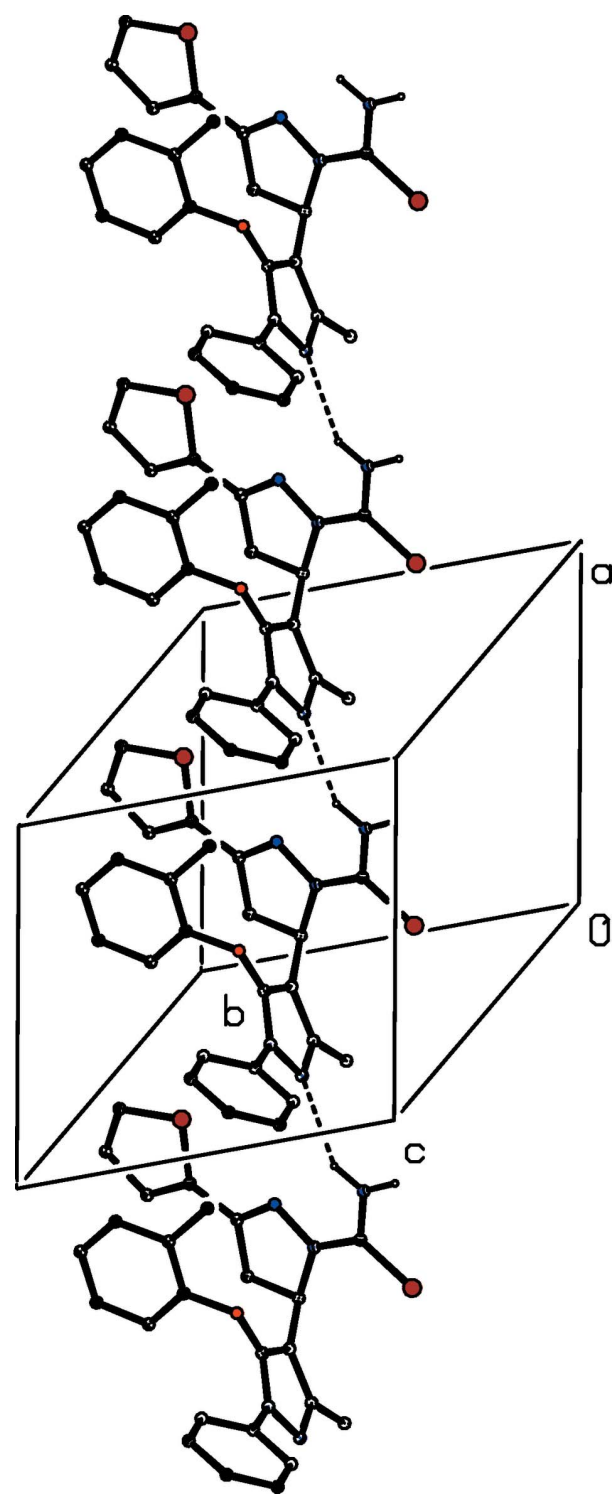

Figure 6

Part of the crystal structure of compound (Ia) showing the formation of a hydrogen-bonded chain running parallel to the [100] direction. Hydrogen bonds are drawn as dashed lines and, for the sake of clarity, the $\mathrm{H}$ atoms which are bonded to $\mathrm{C}$ atoms have been omitted.

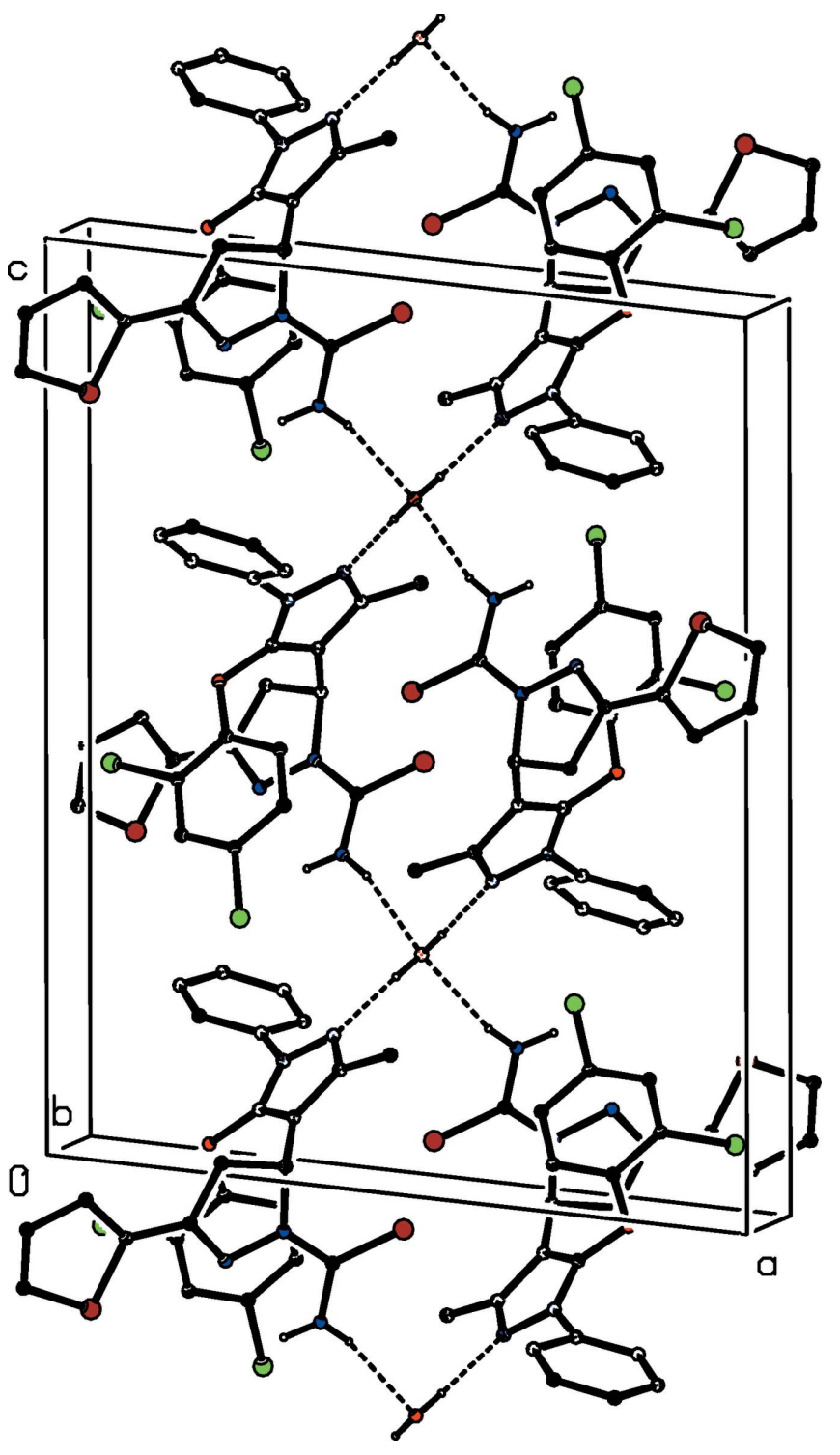

Figure 7

Part of the crystal structure of compound (Ib) showing the formation of a hydrogen-bonded chain of spiro-fused rings running parallel to the [001] direction. Hydrogen bonds are drawn as dashed lines and, for the sake of clarity, the $\mathrm{H}$ atoms which are bonded to $\mathrm{C}$ atoms have been omitted.

this chain the water molecules, which act as double donors in $\mathrm{O}-\mathrm{H} \cdots \mathrm{N}$ hydrogen bonds and double acceptors in $\mathrm{N}-$ $\mathrm{H}$... O hydrogen bonds, are the points of fusion between adjacent rings (Fig. 7).

There are three short intermolecular contacts in the structure of compound (II). That involving atom C13 (Table 1) has a very small $D-\mathrm{H} \cdots A$ angle, and so is unlikely to be structurally significant (Wood et al., 2009), while that involving atom C553 applies only to the minor disorder component, and is absent for the majority of the molecules. The only possible significant interaction is thus that involving atom C54, which links inversion-related pairs of molecules to form a cyclic centrosymmetric motif (Fig. 8). There are no significant hydrogen bonds of any type in the structure of compound (III). 


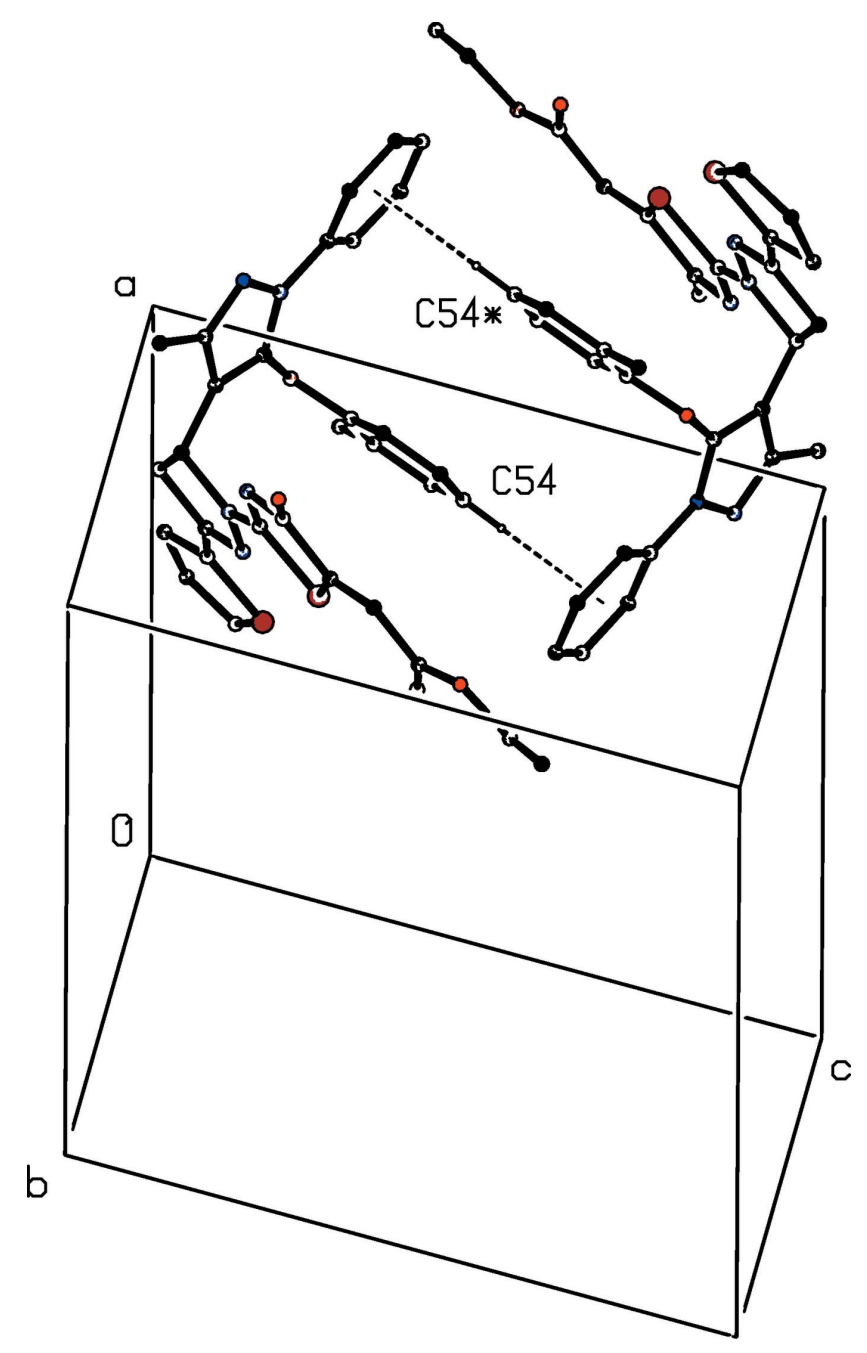

Figure 8

Part of the crystal structure of compound (II) showing the formation of a cyclic centrosymmetric dimer containing $\mathrm{C}-\mathrm{H} \cdots \pi($ arene) hydrogen bonds. For the sake of clarity, the minor disorder components, and the $\mathrm{H}$ atoms not involved in the motif shown have been omitted. The atom marked with an asterisk (*) is at the symmetry position $(2-x,-y, 1-z)$.

\section{Database survey}

Structures have been reported recently for a number of compounds related to those reported here, including precursors and intermediates in the synthetic pathways to compounds (I)-(III). The structures of five examples of 5-aryloxy-3-methyl-1-phenyl-1 $H$-pyrazole-4-carbaldehydes have been reported (Shahani et al., 2011; Vinutha et al., 2014; Glidewell et al., 2019; Kiran Kumar et al., 2019), as have those (Shaibah et al., 2020) of two isostructural chalcones derived from two such carbaldehydes by condensation reactions with 2-acetylthiophene, in each of which the thiophene unit shows the same type of disorder as observed here in compounds (Ia), (Ib), (II) and (III). Structures have also been reported (Cuartas et al., 2017; Kiran Kumar et al., 2019) for several reduced 3,4'-bipyrazoles formed by cyclocondensation reactions between chalcones and hydrazine followed by $\mathrm{N}$ acetylation. However, the only structure reported to date of a product in which the $3,4^{\prime}$-bipyrazole unit is embedded within a group of other cyclic substituents, as in (I)-(III) is that for the methyl ester analogue of (II) (Manju et al., 2019). The original report on this compound provided no crystallographic information other than a molecular structure plot. However, the deposited CIF (CCDC deposition No. 1588961) shows that the reflection data have been subjected to the SQUEEZE procedure (Spek, 2015), although this is not mentioned in the original report. The CIF also shows two sites for the $\mathrm{O}$ atom of the aryloxy unit, $c a 1.28 \AA$ apart with occupancies of $0.843(6)$ and $0.157(6)$ and involving some unexpected geometrical features, although all other atoms are reported as being fully ordered. Hence this structure is unlikely to be entirely correct.

\section{Synthesis and crystallization}

Samples of compounds (Ia), (Ib), (II) and (III) were prepared using the methods previously reported (Manju et al., 2019). Crystals suitable for single-crystal X-ray diffraction were grown by slow evaporation, at ambient temperature and in the presence of air, of solutions in a mixture of ethanol and $\mathrm{N}, \mathrm{N}$ dimethylformamide (initial composition 3:1, v/v).

\section{Refinement}

Crystal data, data collection and structure refinement details are summarized in Table 2. Several bad outlier reflections were omitted from the refinements. i.e. for (Ia) $(\overline{4}, \overline{1}, 18)$; for (Ic) $(1,1,1),(14,0,0),(\overline{15}, 0,6),(\overline{14}, 1,19),(\overline{8}, 9,7)$ and $(\overline{11}, 3,2)$; and for (II) $(\overline{3}, \overline{10}, 2)$ and $(0,5,13)$. All $\mathrm{H}$ atoms, apart from those in the minor disorder components, were located in difference maps. The $\mathrm{H}$ atoms bonded to $\mathrm{C}$ atoms were then treated as riding atoms in geometrically idealized positions with $\mathrm{C}-\mathrm{H}$ distances of $0.93 \AA$ (alkenyl, aromatic and thienyl), $0.96 \AA\left(\mathrm{CH}_{3}\right), 0.97 \AA\left(\mathrm{CH}_{2}\right)$ or $0.98 \AA$ (aliphatic $\left.\mathrm{C}-\mathrm{H}\right)$, and with $U_{\text {iso }}(\mathrm{H})=k U_{\text {eq }}(\mathrm{C})$, where $k=1.5$ for the methyl groups, which were permitted to rotate but not to tilt, and 1.2 for all other $\mathrm{H}$ atoms bonded to $\mathrm{C}$ atoms. For the $\mathrm{H}$ atoms bonded to $\mathrm{N}$ or $\mathrm{O}$ atoms, the atomic coordinates were refined with $U_{\text {iso }}(\mathrm{H})=1.2 \mathrm{U}_{\text {eq }}(\mathrm{N})$ or $1.5 U_{\text {eq }}(\mathrm{O})$, giving the $\mathrm{N}-\mathrm{H}$ and $\mathrm{O}-\mathrm{H}$ distances shown in Table 1. For the minor disorder components, the bonded distances and the 1,3 non-bonded distances were restrained to be the same as the corresponding distances in the major disorder components, subject to s.u. values of 0.01 and $0.02 \AA$, respectively. In addition, the anisotropic displacement parameters associated with pairs of atomic sites occupying essentially the same regions of physical space were constrained to be equal. Subject to these conditions, the occupancies, in the crystals selected for data collection, of the disordered thienyl units refined to 0.866 (3) and 0.134 (3) in (Ia), 0.951 (3) and 0.049 (3) in (Ib), 0.768 (6) and 0.232 (6) in (II), and 0.947 (4) and 0.053 (4) in (III).

\section{Acknowledgements}

CHC thanks the University of Mysore for research facilities. 
Table 2

Experimental details.

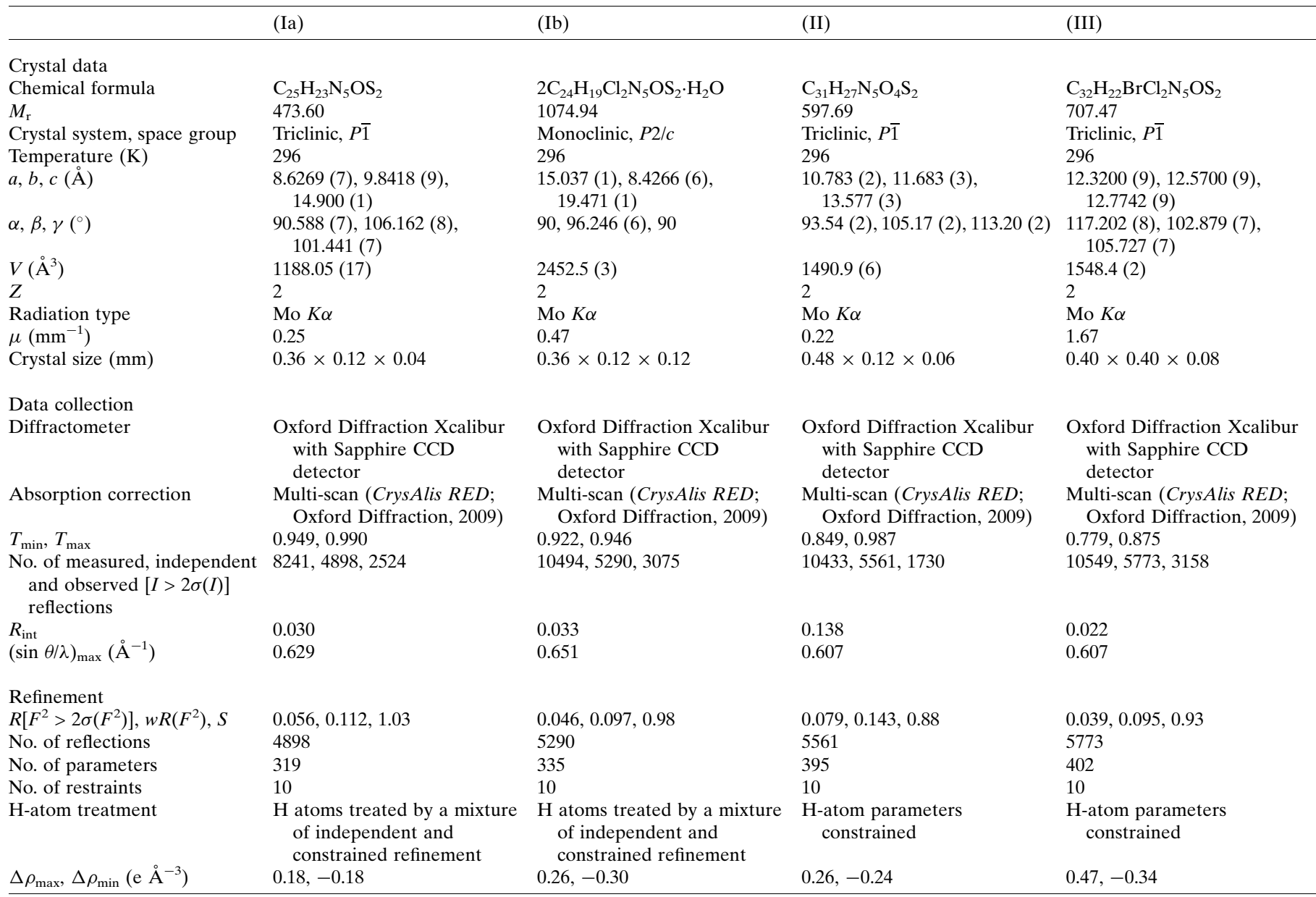

Computer programs: CrysAlis CCD and CrysAlis RED (Oxford Diffraction, 2009), SHELXT (Sheldrick, 2015a), SHELXL2014 (Sheldrick, $2015 b$ ) and PLATON (Spek, 2020).

\section{Funding information}

HSY thanks the University Grants Commission, New Delhi for the award of a BSR Faculty Fellowship for three years.

\section{References}

Bernstein, J., Davis, R. E., Shimoni, L. \& Chang, N.-L. (1995). Angew. Chem. Int. Ed. Engl. 34, 1555-1573.

Bondock, S., Fadaly, W. \& Metwally, M. A. (2010). Eur. J. Med. Chem. 45, 3692-3701.

Cuartas, V., Insuasty, B., Cobo, J. \& Glidewell, C. (2017). Acta Cryst. C73, 784-790.

Etter, M. C. (1990). Acc. Chem. Res. 23, 120-126.

Etter, M. C., MacDonald, J. C. \& Bernstein, J. (1990). Acta Cryst. B46, 256-262.

Girisha, K. S., Kalluraya, B., Narayana, V. \& Padmashree (2010). Eur. J. Med. Chem. 45, 4640-4644.

Glidewell, C., Kalluraya, B., Rathore, R. S. \& Yathirajan, H. S. (2019). CSD Communication (deposition No. 1897876). CCDC, Cambridge, England.

Havrylyuk, D., Roman, O. \& Lesyk, R. (2016). Eur. J. Med. Chem. 113, 145-166.

Isloor, A. M., Kalluraya, B. \& Shetty, P. (2009). Eur. J. Med. Chem. 44, 3784-3787.

Isloor, A. M., Kalluraya, B. \& Pai, S. K. (2010). Eur. J. Med. Chem. 45, 825-830.
Kiran Kumar, H., Yathirajan, H. S., Manju, N., Kalluraya, B., Rathore, R. S. \& Glidewell, C. (2019). Acta Cryst. C75, 768-776.

Manju, N., Kalluraya, B., Asma, Madan Kumar, S., Revanasiddappa, B. \& Chandra (2019). J. Med. Chem. Sci. 2, 101-109.

Oxford Diffraction (2009). CrysAlis CCD and CrysAlis RED. Oxford Diffraction Ltd, Abingdon, England.

Rai, N. S., Kalluraya, B., Lingappa, B., Shenoy, S. \& Puranic, V. G. (2008). Eur. J. Med. Chem. 43, 1715-1720.

Rostom, S. A. F., El-Ashmawy, I. M., Abd El Razik, H. A., Badr, M. H. \& Ashour, H. M. A. (2009). Bioorg. Med. Chem. 17, 882-895.

Shahani, T., Fun, H.-K., Shetty, S. \& Kalluraya, B. (2011). Acta Cryst. E67, o2646.

Shaibah, M. A. E., Yathirajan, H. S., Manju, N., Kalluraya, B., Rathore, R. S. \& Glidewell, C. (2020). Acta Cryst. E76, 48-52.

Sheldrick, G. M. (2015a). Acta Cryst. A71, 3-8.

Sheldrick, G. M. (2015b). Acta Cryst. C71, 3-8.

Spek, A. L. (2015). Acta Cryst. C71, 9-18.

Spek, A. L. (2020). Acta Cryst. E76, 1-11.

Sulthana, S. S., Antony, S. A., Balachandran, C. \& Shafi, S. S. (2015). Bioorg. Med. Chem. Lett. 25, 2753-2757.

Vijesh, A. M., Isloor, A. M., Shetty, P., Sundershan, S. \& Fun, H.-K. (2013). Eur. J. Med. Chem. 62, 410-415.

Vinutha, N., Kumar, S. M., Shobhitha, S., Kalluraya, B., Lokanath, N. K. \& Revannasiddaiah, D. (2014). Acta Cryst. E70, o560.

Wood, P. A., Allen, F. H. \& Pidcock, E. (2009). CrystEngComm, 11, $1563-1571$. 


\section{supporting information}

Acta Cryst. (2021). E77, 335-340 [https://doi.org/10.1107/S2056989021002310]

Reduced 3,4'-bipyrazoles carrying thiophene and thiazole substituents:

structures of two intermediates and two products

Chayanna Harish Chinthal, Hemmige S. Yathirajan, Nagaraja Manju, Balakrishna Kalluraya, Sabine Foro and Christopher Glidewell

Computing details

For all structures, data collection: CrysAlis CCD (Oxford Diffraction, 2009); cell refinement: CrysAlis RED (Oxford Diffraction, 2009); data reduction: CrysAlis RED (Oxford Diffraction, 2009); program(s) used to solve structure:

SHELXT (Sheldrick, 2015a); program(s) used to refine structure: SHELXL2014 (Sheldrick, 2015b); molecular graphics: PLATON (Spek, 2020); software used to prepare material for publication: SHELXL2014 (Sheldrick, 2015b) and PLATON (Spek, 2020).

3'-Methyl-5' -(2-methylphenoxy)-1'-phenyl-5-(thiophen-2-yl)-3,4-dihydro-1'H,2H-3,4'-bipyrazole-2-

carbothioamide (la)

Crystal data

$\mathrm{C}_{25} \mathrm{H}_{23} \mathrm{~N}_{5} \mathrm{OS}_{2}$

$M_{r}=473.60$

Triclinic, $P \overline{1}$

$a=8.6269(7) \AA$

$b=9.8418(9) \AA$

$c=14.900(1) \AA$

$\alpha=90.588(7)^{\circ}$

$\beta=106.162(8)^{\circ}$

$\gamma=101.441(7)^{\circ}$

$V=1188.05(17) \AA^{3}$

Data collection

Oxford Diffraction Xcalibur with Sapphire

$\mathrm{CCD}$ detector diffractometer

Radiation source: Enhance (Mo) X-ray Source

Graphite monochromator

$\omega$ scans

Absorption correction: multi-scan

(CrysAlis RED; Oxford Diffraction, 2009)

$T_{\min }=0.949, T_{\max }=0.990$
$Z=2$

$F(000)=496$

$D_{\mathrm{x}}=1.324 \mathrm{Mg} \mathrm{m}^{-3}$

Mo $K \alpha$ radiation, $\lambda=0.71073 \AA$

Cell parameters from 5098 reflections

$\theta=2.9-27.9^{\circ}$

$\mu=0.25 \mathrm{~mm}^{-1}$

$T=296 \mathrm{~K}$

Needle, yellow

$0.36 \times 0.12 \times 0.04 \mathrm{~mm}$

8241 measured reflections

4898 independent reflections

2524 reflections with $I>2 \sigma(I)$

$R_{\text {int }}=0.030$

$\theta_{\text {max }}=26.6^{\circ}, \theta_{\min }=2.9^{\circ}$

$h=-10 \rightarrow 9$

$k=-10 \rightarrow 12$

$l=-16 \rightarrow 18$ 


\section{Refinement}

Refinement on $F^{2}$

Least-squares matrix: full

$R\left[F^{2}>2 \sigma\left(F^{2}\right)\right]=0.056$

$w R\left(F^{2}\right)=0.112$

$S=1.03$

4898 reflections

319 parameters

10 restraints
Primary atom site location: difference Fourier map

Hydrogen site location: mixed

$\mathrm{H}$ atoms treated by a mixture of independent and constrained refinement

$w=1 /\left[\sigma^{2}\left(F_{\mathrm{o}}^{2}\right)+(0.038 P)^{2}+0.0763 P\right]$ where $P=\left(F_{\mathrm{o}}^{2}+2 F_{\mathrm{c}}^{2}\right) / 3$

$(\Delta / \sigma)_{\max }<0.001$

$\Delta \rho_{\max }=0.18 \mathrm{e} \AA^{-3}$

$\Delta \rho_{\min }=-0.18$ e $\AA^{-3}$

Special details

Experimental. CrysAlis RED, Oxford Diffraction Ltd., 2009 Empirical absorption correction using spherical harmonics, implemented in SCALE3 ABSPACK scaling algorithm.

Geometry. All esds (except the esd in the dihedral angle between two 1.s. planes) are estimated using the full covariance matrix. The cell esds are taken into account individually in the estimation of esds in distances, angles and torsion angles; correlations between esds in cell parameters are only used when they are defined by crystal symmetry. An approximate (isotropic) treatment of cell esds is used for estimating esds involving l.s. planes.

Fractional atomic coordinates and isotropic or equivalent isotropic displacement parameters $\left(\AA^{2}\right)$

\begin{tabular}{llllll}
\hline & $x$ & $y$ & $z$ & $U_{\text {iso }}^{*} / U_{\text {eq }}$ & Occ. $(<1)$ \\
\hline N1 & $-0.0795(2)$ & $0.6663(2)$ & $0.30851(14)$ & $0.0406(6)$ & \\
N2 & $-0.2192(2)$ & $0.6179(2)$ & $0.23518(15)$ & $0.0451(6)$ & \\
C3 & $-0.1670(3)$ & $0.6245(3)$ & $0.15926(18)$ & $0.0421(7)$ & \\
C4 & $0.0047(3)$ & $0.6737(3)$ & $0.18050(17)$ & $0.0384(7)$ & \\
C5 & $0.0541(3)$ & $0.6994(3)$ & $0.27513(17)$ & $0.0384(7)$ & \\
C11 & $-0.0885(3)$ & $0.6605(3)$ & $0.40219(18)$ & $0.0445(7)$ & \\
C12 & $0.0024(3)$ & $0.7654(4)$ & $0.4690(2)$ & $0.0625(9)$ \\
H12 & 0.0687 & 0.8426 & 0.4532 & $0.075^{*}$ \\
C13 & $-0.0067(4)$ & $0.7541(5)$ & $0.5603(2)$ & $0.0816(12)$ & \\
H13 & 0.0563 & 0.8232 & 0.6063 & $0.098^{*}$ \\
C14 & $-0.1073(5)$ & $0.6421(5)$ & $0.5835(2)$ & $0.0838(12)$ & \\
H14 & -0.1123 & 0.6354 & 0.6448 & $0.101^{*}$ \\
C15 & $-0.2004(4)$ & $0.5399(4)$ & $0.5157(3)$ & $0.0757(11)$ & \\
H15 & -0.2704 & 0.4648 & 0.5310 & $0.091^{*}$ \\
C16 & $-0.1904(3)$ & $0.5484(3)$ & $0.4250(2)$ & $0.0565(8)$ \\
H16 & -0.2524 & 0.4784 & 0.3793 & $0.068^{*}$ \\
C31 & $-0.2869(3)$ & $0.5831(3)$ & $0.06491(18)$ & $0.0615(9)$ \\
H31A & -0.2770 & 0.6581 & 0.0249 & $0.092^{*}$ \\
H31B & -0.3970 & 0.5616 & 0.0707 & $0.092^{*}$ \\
H31C & -0.2637 & 0.5027 & 0.0384 & $0.092^{*}$ \\
N41 & $0.4015(2)$ & $0.7247(3)$ & $0.16104(14)$ & $0.0415(6)$ \\
N42 & $0.2565(2)$ & $0.6253(2)$ & $0.14876(14)$ & $0.0393(6)$ \\
C43 & $0.1056(3)$ & $0.6826(3)$ & $0.11269(17)$ & $0.0412(7)$ \\
H43 & 0.0379 & 0.6306 & 0.0538 & $0.049^{*}$ \\
C44 & $0.1805(3)$ & $0.8295(3)$ & $0.09133(18)$ & $0.0496(8)$ \\
H44A & 0.1515 & 0.8403 & 0.0244 & $0.060^{*}$ \\
H44B & 0.1441 & 0.8997 & 0.1218 & $0.060^{*}$ &
\end{tabular}




\begin{tabular}{|c|c|c|c|c|c|}
\hline $\mathrm{C} 45$ & $0.3626(3)$ & 0.8383 & $0.13106(18)$ & $0.0418(7)$ & \\
\hline S421 & $0.09307(9)$ & $0.36681(8)$ & $0.14413(5)$ & 0.0559 & \\
\hline $\mathrm{C} 422$ & 0.2638 & 0.4938 & $0.16724(17)$ & $0.0408(7)$ & \\
\hline N423 & 0.4148 & 0.4679 & $0.20351(18)$ & $0.0538(7)$ & \\
\hline $\mathrm{H} 42 \mathrm{~A}$ & $0.506(3)$ & $0.539(3)$ & $0.2180(18)$ & $0.065 *$ & \\
\hline H42B & $0.426(3)$ & $0.380(3)$ & 0.2139 (19) & $0.065^{*}$ & \\
\hline S451 & $0.69257(13)$ & $0.96287(15)$ & $0.18083(12)$ & $0.0769(5)$ & $0.866(3)$ \\
\hline $\mathrm{C} 452$ & 0.4879 & $0.9623(3)$ & $0.13580(19)$ & $0.0515(8)$ & $0.866(3)$ \\
\hline $\mathrm{C} 453$ & $0.4652(18)$ & $1.0876(10)$ & 0.1098 (14) & $0.0769(15)$ & $0.866(3)$ \\
\hline H453 & 0.3615 & 1.1067 & 0.0830 & $0.092 *$ & $0.866(3)$ \\
\hline $\mathrm{C} 454$ & $0.6146(9)$ & $1.1897(8)$ & $0.1270(14)$ & $0.104(2)$ & $0.866(3)$ \\
\hline H454 & 0.6204 & 1.2821 & 0.1127 & $0.125^{*}$ & $0.866(3)$ \\
\hline $\mathrm{C} 455$ & $0.7456(8)$ & $1.1343(6)$ & $0.1666(8)$ & $0.099(2)$ & $0.866(3)$ \\
\hline H455 & 0.8539 & 1.1848 & 0.1844 & $0.119 *$ & $0.866(3)$ \\
\hline S551 & $0.456(3)$ & 1.1213 (19) & $0.104(3)$ & $0.0769(15)$ & $0.134(3)$ \\
\hline C552 & $0.4879(3)$ & $0.9623(3)$ & $0.13580(19)$ & $0.0515(8)$ & $0.134(3)$ \\
\hline C553 & 0.6476 (19) & $0.960(4)$ & $0.159(4)$ & $0.0769(5)$ & $0.134(3)$ \\
\hline H553 & 0.6877 & 0.8792 & 0.1740 & $0.092 *$ & $0.134(3)$ \\
\hline C554 & $0.751(3)$ & $1.092(4)$ & $0.158(6)$ & $0.099(2)$ & 0.134 (3) \\
\hline H554 & 0.8660 & 1.1083 & 0.1735 & $0.119 *$ & $0.134(3)$ \\
\hline C555 & $0.661(4)$ & $1.189(4)$ & $0.133(10)$ & $0.104(2)$ & $0.134(3)$ \\
\hline H555 & 0.7062 & 1.2828 & 0.1317 & $0.125^{*}$ & $0.134(3)$ \\
\hline O51 & $0.20838(18)$ & 0.73742 (19) & $0.33641(11)$ & $0.0434(5)$ & \\
\hline C51 & 0.2920 & 0.8764 & $0.34104(17)$ & $0.0432(7)$ & \\
\hline C52 & 0.4615 & $0.8993(4)$ & $0.37778(18)$ & $0.0535(8)$ & \\
\hline C53 & $0.5473(5)$ & $1.0365(5)$ & $0.3857(2)$ & $0.0833(13)$ & \\
\hline H53 & 0.6615 & 1.0564 & 0.4102 & $0.100 *$ & \\
\hline C54 & $0.4682(7)$ & $1.1423(5)$ & $0.3583(3)$ & $0.1011(15)$ & \\
\hline H54 & 0.5291 & 1.2330 & 0.3649 & $0.121 *$ & \\
\hline C55 & $0.3006(6)$ & $1.1174(4)$ & $0.3213(3)$ & $0.0882(12)$ & \\
\hline H55 & 0.2478 & 1.1901 & 0.3018 & $0.106^{*}$ & \\
\hline C56 & $0.2104(4)$ & $0.9822(4)$ & $0.3131(2)$ & $0.0626(9)$ & \\
\hline H56 & 0.0961 & 0.9634 & 0.2889 & $0.075^{*}$ & \\
\hline $\mathrm{C} 57$ & $0.5475(4)$ & $0.7841(4)$ & $0.4051(2)$ & $0.0804(11)$ & \\
\hline H57A & 0.6602 & 0.8211 & 0.4398 & $0.121 *$ & \\
\hline H57B & 0.5441 & 0.7310 & 0.3500 & $0.121 *$ & \\
\hline $\mathrm{H} 57 \mathrm{C}$ & 0.4940 & 0.7253 & 0.4435 & $0.121^{*}$ & \\
\hline
\end{tabular}

Atomic displacement parameters $\left(\AA^{2}\right)$

\begin{tabular}{lllllll}
\hline & $U^{11}$ & $U^{22}$ & $U^{33}$ & $U^{12}$ & $U^{13}$ & $U^{23}$ \\
\hline $\mathrm{N} 1$ & $0.0280(12)$ & $0.0587(17)$ & $0.0333(13)$ & $0.0070(10)$ & $0.0073(10)$ & $0.0036(11)$ \\
$\mathrm{N} 2$ & $0.0269(12)$ & $0.0642(18)$ & $0.0403(13)$ & $0.0050(11)$ & $0.0063(11)$ & $0.0041(11)$ \\
$\mathrm{C} 3$ & $0.0276(14)$ & $0.057(2)$ & $0.0382(16)$ & $0.0036(12)$ & $0.0073(12)$ & $0.0040(13)$ \\
$\mathrm{C} 4$ & $0.0265(14)$ & $0.0520(19)$ & $0.0335(16)$ & $0.0026(12)$ & $0.0072(12)$ & $0.0031(13)$ \\
$\mathrm{C} 5$ & $0.0237(14)$ & $0.0491(19)$ & $0.0400(16)$ & $0.0047(12)$ & $0.0073(12)$ & $0.0048(13)$ \\
C11 & $0.0319(15)$ & $0.070(2)$ & $0.0363(17)$ & $0.0195(14)$ & $0.0112(13)$ & $0.0073(15)$ \\
C12 & $0.0516(19)$ & $0.089(3)$ & $0.049(2)$ & $0.0129(17)$ & $0.0179(16)$ & $-0.0042(18)$
\end{tabular}




\begin{tabular}{|c|c|c|c|c|c|c|}
\hline $\mathrm{C} 13$ & $0.071(2)$ & $0.129(4)$ & $0.049(2)$ & $0.029(2)$ & $0.0175(19)$ & $-0.011(2)$ \\
\hline $\mathrm{C} 14$ & 0.085 & $0.143(4)$ & $0.047(2)$ & $0.059(3)$ & $0.031(2)$ & $0.023(2)$ \\
\hline $\mathrm{C} 15$ & $0.073(2)$ & $0.105(3)$ & $0.069(2)$ & $0.037(2)$ & $0.038(2)$ & $0.039(2)$ \\
\hline $\mathrm{C} 16$ & $0.0508(18)$ & $0.072(2)$ & $0.053(2)$ & $0.0205(16)$ & $0.0208(15)$ & $0.0169(16)$ \\
\hline $\mathrm{C} 31$ & $0.0324(16)$ & $0.095(3)$ & $0.0440(18)$ & $-0.0081(15)$ & $0.0050(14)$ & $-0.0017(17)$ \\
\hline N41 & $0.0288(12)$ & $0.0448(16)$ & $0.0471(14)$ & $-0.0054(11)$ & $0.0141(10)$ & $-0.0002(12)$ \\
\hline N42 & $0.0259(12)$ & $0.0429(16)$ & $0.0468(14)$ & $-0.0018(10)$ & $0.0132(10)$ & $0.0027(11)$ \\
\hline $\mathrm{C} 43$ & $0.0313(15)$ & $0.054(2)$ & $0.0342(15)$ & $0.0018(13)$ & $0.0073(12)$ & 0.0049 (13) \\
\hline $\mathrm{C} 44$ & $0.0437(17)$ & $0.060(2)$ & $0.0465(17)$ & $0.0070(14)$ & $0.0172(14)$ & $0.0109(15)$ \\
\hline $\mathrm{C} 45$ & $0.0369(16)$ & $0.049(2)$ & $0.0376(16)$ & $-0.0023(14)$ & $0.0154(13)$ & $0.0021(14)$ \\
\hline S421 & $0.0477(5)$ & $0.0563(6)$ & $0.0525(5)$ & $-0.0151(4)$ & $0.0143(4)$ & $0.0049(4)$ \\
\hline $\mathrm{C} 422$ & $0.0387(17)$ & $0.049(2)$ & $0.0340(16)$ & $-0.0004(14)$ & $0.0155(13)$ & $0.0007(13)$ \\
\hline N423 & $0.0410(15)$ & $0.0452(18)$ & $0.0733(18)$ & $0.0051(13)$ & $0.0161(14)$ & $0.0093(15)$ \\
\hline S451 & $0.0458(7)$ & $0.0803(9)$ & $0.0897(12)$ & $-0.0215(6)$ & $0.0194(7)$ & $0.0077(7)$ \\
\hline $\mathrm{C} 452$ & $0.0507(18)$ & $0.050(2)$ & $0.0530(19)$ & $-0.0039(15)$ & $0.0241(15)$ & $0.0042(15)$ \\
\hline $\mathrm{C} 453$ & $0.095(3)$ & $0.050(5)$ & $0.095(3)$ & $0.014(4)$ & $0.041(2)$ & $0.022(5)$ \\
\hline $\mathrm{C} 454$ & $0.144(6)$ & $0.050(3)$ & $0.120(5)$ & $-0.023(4)$ & $0.071(7)$ & $0.013(3)$ \\
\hline $\mathrm{C} 455$ & $0.097(3)$ & $0.076(5)$ & $0.106(4)$ & $-0.048(3)$ & $0.046(3)$ & $-0.013(5)$ \\
\hline S551 & $0.095(3)$ & $0.050(5)$ & $0.095(3)$ & $0.014(4)$ & $0.041(2)$ & $0.022(5)$ \\
\hline C552 & $0.0507(18)$ & $0.050(2)$ & $0.0530(19)$ & $-0.0039(15)$ & $0.0241(15)$ & $0.0042(15)$ \\
\hline C553 & $0.0458(7)$ & $0.0803(9)$ & $0.0897(12)$ & $-0.0215(6)$ & $0.0194(7)$ & $0.0077(7)$ \\
\hline C554 & $0.097(3)$ & $0.076(5)$ & $0.106(4)$ & -0.048 & $0.046(3)$ & $-0.013(5)$ \\
\hline C555 & $0.144(6)$ & $0.050(3)$ & $0.120(5)$ & -0.023 & $0.071(7)$ & $0.013(3)$ \\
\hline O51 & $0.0265(10)$ & $0.0521(13)$ & $0.0445(11)$ & $0.0054(8)$ & $0.0009(8)$ & $0.0032(9)$ \\
\hline C51 & $0.0373(16)$ & $0.050(2)$ & $0.0386(16)$ & $0.0002(14)$ & $0.0115(13)$ & $-0.0033(14)$ \\
\hline C52 & $0.0380(17)$ & 0.075 & $0.0406(17)$ & $0.0016(16)$ & $0.0073(14)$ & $-0.0046(16)$ \\
\hline $\mathrm{C} 53$ & $0.064(2)$ & $0.100(4)$ & $0.065(2)$ & $-0.028(2)$ & $0.0160(19)$ & -0.015 (2) \\
\hline C54 & $0.129(4)$ & $0.073(4)$ & 0.085 & $-0.032(3)$ & $0.043(3)$ & -0.017 \\
\hline C55 & $0.137(4)$ & $0.054(3)$ & $0.083(3)$ & $0.018(3)$ & $0.048(3)$ & $0.004(2)$ \\
\hline C56 & $0.069(2)$ & $0.057(3)$ & $0.066(2)$ & $0.0164(19)$ & $0.0243(18)$ & $0.0042(18)$ \\
\hline C57 & $0.0410(19)$ & $0.119(4)$ & $0.072(2)$ & $0.018(2)$ & $0.0016(17)$ & $0.001(2)$ \\
\hline
\end{tabular}

Geometric parameters $\left(A,{ }^{\circ}\right)$

\begin{tabular}{llll}
\hline $\mathrm{N} 1-\mathrm{C} 5$ & $1.362(3)$ & $\mathrm{S} 421-\mathrm{C} 422$ & $1.682(3)$ \\
$\mathrm{N} 1-\mathrm{N} 2$ & $1.379(2)$ & $\mathrm{C} 422-\mathrm{N} 423$ & $1.341(3)$ \\
$\mathrm{N} 1-\mathrm{C} 11$ & $1.421(3)$ & $\mathrm{N} 423-\mathrm{H} 42 \mathrm{~A}$ & $0.91(3)$ \\
$\mathrm{N} 2-\mathrm{C} 3$ & $1.326(3)$ & $\mathrm{N} 423-\mathrm{H} 42 \mathrm{~B}$ & $0.90(3)$ \\
$\mathrm{C} 3-\mathrm{C} 4$ & $1.407(3)$ & $\mathrm{S} 451-\mathrm{C} 455$ & $1.690(6)$ \\
$\mathrm{C} 3-\mathrm{C} 31$ & $\mathrm{~S} 451-\mathrm{C} 452$ & $1.704(3)$ \\
$\mathrm{C} 4-\mathrm{C} 5$ & $1.491(3)$ & $\mathrm{C} 452-\mathrm{C} 453$ & $1.331(8)$ \\
$\mathrm{C} 4-\mathrm{C} 43$ & $1.360(3)$ & $\mathrm{C} 453-\mathrm{C} 454$ & $1.426(13)$ \\
$\mathrm{C} 5-\mathrm{O} 51$ & $1.499(3)$ & $\mathrm{C} 453-\mathrm{H} 453$ & 0.9300 \\
$\mathrm{C} 11-\mathrm{C} 12$ & $1.366(3)$ & $\mathrm{C} 454-\mathrm{C} 455$ & $1.343(7)$ \\
$\mathrm{C} 11-\mathrm{C} 16$ & $1.376(4)$ & $\mathrm{C} 454-\mathrm{H} 454$ & 0.9300 \\
$\mathrm{C} 12-\mathrm{C} 13$ & $1.376(4)$ & $\mathrm{C} 455-\mathrm{H} 455$ & 0.9300 \\
$\mathrm{C} 12-\mathrm{H} 12$ & $1.389(4)$ & $\mathrm{S} 551-\mathrm{C} 555$ & $1.690(12)$ \\
$\mathrm{C} 13-\mathrm{C} 14$ & 0.9300 & $\mathrm{C} 553-\mathrm{C} 554$ & $1.428(16)$
\end{tabular}




\begin{tabular}{|c|c|c|c|}
\hline $\mathrm{C} 13-\mathrm{H} 13$ & 0.9300 & C553-H553 & 0.9300 \\
\hline $\mathrm{C} 14-\mathrm{C} 15$ & $1.372(5)$ & C554-C555 & $1.344(12)$ \\
\hline $\mathrm{C} 14-\mathrm{H} 14$ & 0.9300 & С554-H554 & 0.9300 \\
\hline $\mathrm{C} 15-\mathrm{C} 16$ & $1.382(4)$ & C555-H555 & 0.9300 \\
\hline $\mathrm{C} 15-\mathrm{H} 15$ & 0.9300 & $\mathrm{O} 51-\mathrm{C} 51$ & $1.406(3)$ \\
\hline $\mathrm{C} 16-\mathrm{H} 16$ & 0.9300 & $\mathrm{C} 51-\mathrm{C} 56$ & $1.374(4)$ \\
\hline $\mathrm{C} 31-\mathrm{H} 31 \mathrm{~A}$ & 0.9600 & C51-C52 & $1.382(4)$ \\
\hline $\mathrm{C} 31-\mathrm{H} 31 \mathrm{~B}$ & 0.9600 & $\mathrm{C} 52-\mathrm{C} 53$ & $1.392(5)$ \\
\hline $\mathrm{C} 31-\mathrm{H} 31 \mathrm{C}$ & 0.9600 & $\mathrm{C} 52-\mathrm{C} 57$ & $1.475(4)$ \\
\hline $\mathrm{N} 41-\mathrm{C} 45$ & $1.280(3)$ & $\mathrm{C} 53-\mathrm{C} 54$ & $1.361(5)$ \\
\hline $\mathrm{N} 41-\mathrm{N} 42$ & $1.392(3)$ & $\mathrm{C} 53-\mathrm{H} 53$ & 0.9300 \\
\hline $\mathrm{N} 42-\mathrm{C} 422$ & $1.336(3)$ & $\mathrm{C} 54-\mathrm{C} 55$ & $1.366(5)$ \\
\hline $\mathrm{N} 42-\mathrm{C} 43$ & $1.487(3)$ & C54-H54 & 0.9300 \\
\hline $\mathrm{C} 43-\mathrm{C} 44$ & $1.537(3)$ & $\mathrm{C} 55-\mathrm{C} 56$ & $1.387(4)$ \\
\hline $\mathrm{C} 43-\mathrm{H} 43$ & 0.9800 & C55-H55 & 0.9300 \\
\hline $\mathrm{C} 44-\mathrm{C} 45$ & $1.501(3)$ & C56-H56 & 0.9300 \\
\hline $\mathrm{C} 44-\mathrm{H} 44 \mathrm{~A}$ & 0.9700 & C57-H57A & 0.9600 \\
\hline $\mathrm{C} 44-\mathrm{H} 44 \mathrm{~B}$ & 0.9700 & C57-H57B & 0.9600 \\
\hline $\mathrm{C} 45-\mathrm{C} 452$ & $1.447(4)$ & C57-H57C & 0.9600 \\
\hline $\mathrm{C} 5-\mathrm{N} 1-\mathrm{N} 2$ & $109.56(19)$ & $\mathrm{N} 41-\mathrm{C} 45-\mathrm{C} 44$ & $114.4(2)$ \\
\hline $\mathrm{C} 5-\mathrm{N} 1-\mathrm{C} 11$ & $130.2(2)$ & $\mathrm{C} 452-\mathrm{C} 45-\mathrm{C} 44$ & $124.5(3)$ \\
\hline $\mathrm{N} 2-\mathrm{N} 1-\mathrm{C} 11$ & $119.8(2)$ & $\mathrm{N} 42-\mathrm{C} 422-\mathrm{N} 423$ & $116.4(2)$ \\
\hline $\mathrm{C} 3-\mathrm{N} 2-\mathrm{N} 1$ & $105.00(18)$ & $\mathrm{N} 42-\mathrm{C} 422-\mathrm{S} 421$ & $121.8(2)$ \\
\hline $\mathrm{N} 2-\mathrm{C} 3-\mathrm{C} 4$ & $112.4(2)$ & $\mathrm{N} 423-\mathrm{C} 422-\mathrm{S} 421$ & $121.7(2)$ \\
\hline $\mathrm{N} 2-\mathrm{C} 3-\mathrm{C} 31$ & $120.2(2)$ & $\mathrm{C} 422-\mathrm{N} 423-\mathrm{H} 42 \mathrm{~A}$ & $120.3(18)$ \\
\hline $\mathrm{C} 4-\mathrm{C} 3-\mathrm{C} 31$ & $127.4(2)$ & $\mathrm{C} 422-\mathrm{N} 423-\mathrm{H} 42 \mathrm{~B}$ & $119.2(18)$ \\
\hline $\mathrm{C} 5-\mathrm{C} 4-\mathrm{C} 3$ & $103.8(2)$ & $\mathrm{H} 42 \mathrm{~A}-\mathrm{N} 423-\mathrm{H} 42 \mathrm{~B}$ & $121(3)$ \\
\hline $\mathrm{C} 5-\mathrm{C} 4-\mathrm{C} 43$ & $129.7(2)$ & $\mathrm{C} 455-\mathrm{S} 451-\mathrm{C} 452$ & $91.8(3)$ \\
\hline $\mathrm{C} 3-\mathrm{C} 4-\mathrm{C} 43$ & $126.3(2)$ & $\mathrm{C} 453-\mathrm{C} 452-\mathrm{C} 45$ & $127.5(7)$ \\
\hline $\mathrm{C} 4-\mathrm{C} 5-\mathrm{N} 1$ & $109.2(2)$ & $\mathrm{C} 453-\mathrm{C} 452-\mathrm{S} 451$ & $111.0(6)$ \\
\hline $\mathrm{C} 4-\mathrm{C} 5-\mathrm{O} 51$ & $130.8(2)$ & $\mathrm{C} 45-\mathrm{C} 452-\mathrm{S} 451$ & $121.5(3)$ \\
\hline $\mathrm{N} 1-\mathrm{C} 5-\mathrm{O} 51$ & $119.7(2)$ & $\mathrm{C} 452-\mathrm{C} 453-\mathrm{C} 454$ & $113.8(8)$ \\
\hline $\mathrm{C} 12-\mathrm{C} 11-\mathrm{C} 16$ & $120.5(3)$ & $\mathrm{C} 452-\mathrm{C} 453-\mathrm{H} 453$ & 123.1 \\
\hline $\mathrm{C} 12-\mathrm{C} 11-\mathrm{N} 1$ & $120.6(3)$ & $\mathrm{C} 454-\mathrm{C} 453-\mathrm{H} 453$ & 123.1 \\
\hline $\mathrm{C} 16-\mathrm{C} 11-\mathrm{N} 1$ & $118.8(3)$ & $\mathrm{C} 455-\mathrm{C} 454-\mathrm{C} 453$ & $110.7(6)$ \\
\hline $\mathrm{C} 11-\mathrm{C} 12-\mathrm{C} 13$ & $118.8(3)$ & $\mathrm{C} 455-\mathrm{C} 454-\mathrm{H} 454$ & 124.7 \\
\hline $\mathrm{C} 11-\mathrm{C} 12-\mathrm{H} 12$ & 120.6 & $\mathrm{C} 453-\mathrm{C} 454-\mathrm{H} 454$ & 124.7 \\
\hline $\mathrm{C} 13-\mathrm{C} 12-\mathrm{H} 12$ & 120.6 & $\mathrm{C} 454-\mathrm{C} 455-\mathrm{S} 451$ & $112.7(5)$ \\
\hline $\mathrm{C} 14-\mathrm{C} 13-\mathrm{C} 12$ & $120.8(3)$ & $\mathrm{C} 454-\mathrm{C} 455-\mathrm{H} 455$ & 123.6 \\
\hline $\mathrm{C} 14-\mathrm{C} 13-\mathrm{H} 13$ & 119.6 & $\mathrm{~S} 451-\mathrm{C} 455-\mathrm{H} 455$ & 123.6 \\
\hline $\mathrm{C} 12-\mathrm{C} 13-\mathrm{H} 13$ & 119.6 & $\mathrm{C} 554-\mathrm{C} 553-\mathrm{H} 553$ & 123.2 \\
\hline $\mathrm{C} 15-\mathrm{C} 14-\mathrm{C} 13$ & $119.8(3)$ & $\mathrm{C} 555-\mathrm{C} 554-\mathrm{C} 553$ & $110.8(13)$ \\
\hline $\mathrm{C} 15-\mathrm{C} 14-\mathrm{H} 14$ & 120.1 & $\mathrm{C} 555-\mathrm{C} 554-\mathrm{H} 554$ & 124.6 \\
\hline $\mathrm{C} 13-\mathrm{C} 14-\mathrm{H} 14$ & 120.1 & C553-C554-H554 & 124.6 \\
\hline $\mathrm{C} 14-\mathrm{C} 15-\mathrm{C} 16$ & $120.1(3)$ & $\mathrm{C} 554-\mathrm{C} 555-\mathrm{S} 551$ & $112.2(12)$ \\
\hline $\mathrm{C} 14-\mathrm{C} 15-\mathrm{H} 15$ & 119.9 & $\mathrm{C} 554-\mathrm{C} 555-\mathrm{H} 555$ & 123.9 \\
\hline $\mathrm{C} 16-\mathrm{C} 15-\mathrm{H} 15$ & 119.9 & S551-C555-H555 & 123.9 \\
\hline
\end{tabular}




\begin{tabular}{|c|c|c|c|}
\hline $\mathrm{C} 11-\mathrm{C} 16-\mathrm{C} 15$ & $119.9(3)$ & $\mathrm{C} 5-\mathrm{O} 51-\mathrm{C} 51$ & $117.9(2)$ \\
\hline $\mathrm{C} 11-\mathrm{C} 16-\mathrm{H} 16$ & 120.0 & $\mathrm{C} 56-\mathrm{C} 51-\mathrm{C} 52$ & $122.6(3)$ \\
\hline $\mathrm{C} 15-\mathrm{C} 16-\mathrm{H} 16$ & 120.0 & $\mathrm{C} 56-\mathrm{C} 51-\mathrm{O} 51$ & $122.3(2)$ \\
\hline $\mathrm{C} 3-\mathrm{C} 31-\mathrm{H} 31 \mathrm{~A}$ & 109.5 & $\mathrm{C} 52-\mathrm{C} 51-\mathrm{O} 51$ & $115.1(3)$ \\
\hline $\mathrm{C} 3-\mathrm{C} 31-\mathrm{H} 31 \mathrm{~B}$ & 109.5 & $\mathrm{C} 51-\mathrm{C} 52-\mathrm{C} 53$ & $116.4(3)$ \\
\hline $\mathrm{H} 31 \mathrm{~A}-\mathrm{C} 31-\mathrm{H} 31 \mathrm{~B}$ & 109.5 & $\mathrm{C} 51-\mathrm{C} 52-\mathrm{C} 57$ & $121.8(3)$ \\
\hline $\mathrm{C} 3-\mathrm{C} 31-\mathrm{H} 31 \mathrm{C}$ & 109.5 & $\mathrm{C} 53-\mathrm{C} 52-\mathrm{C} 57$ & $121.7(3)$ \\
\hline $\mathrm{H} 31 \mathrm{~A}-\mathrm{C} 31-\mathrm{H} 31 \mathrm{C}$ & 109.5 & $\mathrm{C} 54-\mathrm{C} 53-\mathrm{C} 52$ & $121.7(4)$ \\
\hline $\mathrm{H} 31 \mathrm{~B}-\mathrm{C} 31-\mathrm{H} 31 \mathrm{C}$ & 109.5 & $\mathrm{C} 54-\mathrm{C} 53-\mathrm{H} 53$ & 119.2 \\
\hline $\mathrm{C} 45-\mathrm{N} 41-\mathrm{N} 42$ & $108.1(2)$ & $\mathrm{C} 52-\mathrm{C} 53-\mathrm{H} 53$ & 119.2 \\
\hline $\mathrm{C} 422-\mathrm{N} 42-\mathrm{N} 41$ & $119.9(2)$ & $\mathrm{C} 53-\mathrm{C} 54-\mathrm{C} 55$ & $121.0(4)$ \\
\hline $\mathrm{C} 422-\mathrm{N} 42-\mathrm{C} 43$ & $127.2(2)$ & $\mathrm{C} 53-\mathrm{C} 54-\mathrm{H} 54$ & 119.5 \\
\hline $\mathrm{N} 41-\mathrm{N} 42-\mathrm{C} 43$ & $112.9(2)$ & $\mathrm{C} 55-\mathrm{C} 54-\mathrm{H} 54$ & 119.5 \\
\hline $\mathrm{N} 42-\mathrm{C} 43-\mathrm{C} 4$ & $112.3(2)$ & $\mathrm{C} 54-\mathrm{C} 55-\mathrm{C} 56$ & $119.2(4)$ \\
\hline $\mathrm{N} 42-\mathrm{C} 43-\mathrm{C} 44$ & $101.01(19)$ & $\mathrm{C} 54-\mathrm{C} 55-\mathrm{H} 55$ & 120.4 \\
\hline $\mathrm{C} 4-\mathrm{C} 43-\mathrm{C} 44$ & $116.4(2)$ & $\mathrm{C} 56-\mathrm{C} 55-\mathrm{H} 55$ & 120.4 \\
\hline $\mathrm{N} 42-\mathrm{C} 43-\mathrm{H} 43$ & 108.9 & $\mathrm{C} 51-\mathrm{C} 56-\mathrm{C} 55$ & $119.2(3)$ \\
\hline $\mathrm{C} 4-\mathrm{C} 43-\mathrm{H} 43$ & 108.9 & $\mathrm{C} 51-\mathrm{C} 56-\mathrm{H} 56$ & 120.4 \\
\hline $\mathrm{C} 44-\mathrm{C} 43-\mathrm{H} 43$ & 108.9 & $\mathrm{C} 55-\mathrm{C} 56-\mathrm{H} 56$ & 120.4 \\
\hline $\mathrm{C} 45-\mathrm{C} 44-\mathrm{C} 43$ & $102.9(2)$ & C52-C57-H57A & 109.5 \\
\hline $\mathrm{C} 45-\mathrm{C} 44-\mathrm{H} 44 \mathrm{~A}$ & 111.2 & C52-C57-H57B & 109.5 \\
\hline $\mathrm{C} 43-\mathrm{C} 44-\mathrm{H} 44 \mathrm{~A}$ & 111.2 & $\mathrm{H} 57 \mathrm{~A}-\mathrm{C} 57-\mathrm{H} 57 \mathrm{~B}$ & 109.5 \\
\hline $\mathrm{C} 45-\mathrm{C} 44-\mathrm{H} 44 \mathrm{~B}$ & 111.2 & $\mathrm{C} 52-\mathrm{C} 57-\mathrm{H} 57 \mathrm{C}$ & 109.5 \\
\hline $\mathrm{C} 43-\mathrm{C} 44-\mathrm{H} 44 \mathrm{~B}$ & 111.2 & $\mathrm{H} 57 \mathrm{~A}-\mathrm{C} 57-\mathrm{H} 57 \mathrm{C}$ & 109.5 \\
\hline $\mathrm{H} 44 \mathrm{~A}-\mathrm{C} 44-\mathrm{H} 44 \mathrm{~B}$ & 109.1 & $\mathrm{H} 57 \mathrm{~B}-\mathrm{C} 57-\mathrm{H} 57 \mathrm{C}$ & 109.5 \\
\hline $\mathrm{N} 41-\mathrm{C} 45-\mathrm{C} 452$ & $121.1(3)$ & & \\
\hline $\mathrm{C} 5-\mathrm{N} 1-\mathrm{N} 2-\mathrm{C} 3$ & $-0.7(3)$ & $\mathrm{N} 42-\mathrm{C} 43-\mathrm{C} 44-\mathrm{C} 45$ & $-7.5(2)$ \\
\hline $\mathrm{C} 11-\mathrm{N} 1-\mathrm{N} 2-\mathrm{C} 3$ & $-173.9(2)$ & $\mathrm{C} 4-\mathrm{C} 43-\mathrm{C} 44-\mathrm{C} 45$ & $114.3(2)$ \\
\hline $\mathrm{N} 1-\mathrm{N} 2-\mathrm{C} 3-\mathrm{C} 4$ & $1.1(3)$ & $\mathrm{N} 42-\mathrm{N} 41-\mathrm{C} 45-\mathrm{C} 452$ & $179.1(2)$ \\
\hline $\mathrm{N} 1-\mathrm{N} 2-\mathrm{C} 3-\mathrm{C} 31$ & $-178.5(2)$ & $\mathrm{N} 42-\mathrm{N} 41-\mathrm{C} 45-\mathrm{C} 44$ & $-1.3(3)$ \\
\hline $\mathrm{N} 2-\mathrm{C} 3-\mathrm{C} 4-\mathrm{C} 5$ & $-1.1(3)$ & $\mathrm{C} 43-\mathrm{C} 44-\mathrm{C} 45-\mathrm{N} 41$ & $6.1(3)$ \\
\hline $\mathrm{C} 31-\mathrm{C} 3-\mathrm{C} 4-\mathrm{C} 5$ & $178.5(3)$ & $\mathrm{C} 43-\mathrm{C} 44-\mathrm{C} 45-\mathrm{C} 452$ & $-174.4(2)$ \\
\hline $\mathrm{N} 2-\mathrm{C} 3-\mathrm{C} 4-\mathrm{C} 43$ & $174.5(3)$ & $\mathrm{N} 41-\mathrm{N} 42-\mathrm{C} 422-\mathrm{N} 423$ & $4.6(3)$ \\
\hline $\mathrm{C} 31-\mathrm{C} 3-\mathrm{C} 4-\mathrm{C} 43$ & $-5.8(5)$ & $\mathrm{C} 43-\mathrm{N} 42-\mathrm{C} 422-\mathrm{N} 423$ & $-177.0(2)$ \\
\hline $\mathrm{C} 3-\mathrm{C} 4-\mathrm{C} 5-\mathrm{N} 1$ & $0.6(3)$ & $\mathrm{N} 41-\mathrm{N} 42-\mathrm{C} 422-\mathrm{S} 421$ & $-174.18(16)$ \\
\hline $\mathrm{C} 43-\mathrm{C} 4-\mathrm{C} 5-\mathrm{N} 1$ & $-174.8(3)$ & $\mathrm{C} 43-\mathrm{N} 42-\mathrm{C} 422-\mathrm{S} 421$ & $4.2(3)$ \\
\hline $\mathrm{C} 3-\mathrm{C} 4-\mathrm{C} 5-\mathrm{O} 51$ & $173.7(3)$ & $\mathrm{N} 41-\mathrm{C} 45-\mathrm{C} 452-\mathrm{C} 453$ & $-178.0(11)$ \\
\hline $\mathrm{C} 43-\mathrm{C} 4-\mathrm{C} 5-\mathrm{O} 51$ & $-1.7(5)$ & $\mathrm{C} 44-\mathrm{C} 45-\mathrm{C} 452-\mathrm{C} 453$ & $2.5(12)$ \\
\hline $\mathrm{N} 2-\mathrm{N} 1-\mathrm{C} 5-\mathrm{C} 4$ & $0.0(3)$ & $\mathrm{N} 41-\mathrm{C} 45-\mathrm{C} 452-\mathrm{S} 451$ & $0.3(4)$ \\
\hline $\mathrm{C} 11-\mathrm{N} 1-\mathrm{C} 5-\mathrm{C} 4$ & $172.3(3)$ & $\mathrm{C} 44-\mathrm{C} 45-\mathrm{C} 452-\mathrm{S} 451$ & $-179.2(2)$ \\
\hline $\mathrm{N} 2-\mathrm{N} 1-\mathrm{C} 5-\mathrm{O} 51$ & $-174.0(2)$ & $\mathrm{C} 455-\mathrm{S} 451-\mathrm{C} 452-\mathrm{C} 453$ & $1.4(10)$ \\
\hline $\mathrm{C} 11-\mathrm{N} 1-\mathrm{C} 5-\mathrm{O} 51$ & $-1.7(4)$ & $\mathrm{C} 455-\mathrm{S} 451-\mathrm{C} 452-\mathrm{C} 45$ & $-177.2(4)$ \\
\hline $\mathrm{C} 5-\mathrm{N} 1-\mathrm{C} 11-\mathrm{C} 12$ & $45.3(4)$ & $\mathrm{C} 45-\mathrm{C} 452-\mathrm{C} 453-\mathrm{C} 454$ & $177.6(11)$ \\
\hline $\mathrm{N} 2-\mathrm{N} 1-\mathrm{C} 11-\mathrm{C} 12$ & $-143.1(3)$ & $\mathrm{S} 451-\mathrm{C} 452-\mathrm{C} 453-\mathrm{C} 454$ & $-0.9(18)$ \\
\hline $\mathrm{C} 5-\mathrm{N} 1-\mathrm{C} 11-\mathrm{C} 16$ & $-135.0(3)$ & $\mathrm{C} 452-\mathrm{C} 453-\mathrm{C} 454-\mathrm{C} 455$ & $0(2)$ \\
\hline $\mathrm{N} 2-\mathrm{N} 1-\mathrm{C} 11-\mathrm{C} 16$ & $36.6(4)$ & $\mathrm{C} 453-\mathrm{C} 454-\mathrm{C} 455-\mathrm{S} 451$ & $1.4(18)$ \\
\hline $\mathrm{C} 16-\mathrm{C} 11-\mathrm{C} 12-\mathrm{C} 13$ & $2.0(5)$ & $\mathrm{C} 452-\mathrm{S} 451-\mathrm{C} 455-\mathrm{C} 454$ & $-1.6(11)$ \\
\hline
\end{tabular}




$\begin{array}{llll}\mathrm{N} 1-\mathrm{C} 11-\mathrm{C} 12-\mathrm{C} 13 & -178.3(3) & \mathrm{C} 553-\mathrm{C} 554-\mathrm{C} 555-\mathrm{S} 551 & -4(13) \\ \mathrm{C} 11-\mathrm{C} 12-\mathrm{C} 13-\mathrm{C} 14 & -1.6(5) & \mathrm{C} 4-\mathrm{C} 5-\mathrm{O} 51-\mathrm{C} 51 & 76.6(4) \\ \mathrm{C} 12-\mathrm{C} 13-\mathrm{C} 14-\mathrm{C} 15 & -0.1(6) & \mathrm{N} 1-\mathrm{C} 5-\mathrm{O} 51-\mathrm{C} 51 & -110.9(3) \\ \mathrm{C} 13-\mathrm{C} 14-\mathrm{C} 15-\mathrm{C} 16 & 1.3(5) & \mathrm{C} 5-\mathrm{O} 51-\mathrm{C} 51-\mathrm{C} 56 & 22.5(3) \\ \mathrm{C} 12-\mathrm{C} 11-\mathrm{C} 16-\mathrm{C} 15 & -0.8(4) & \mathrm{C} 5-\mathrm{O} 51-\mathrm{C} 51-\mathrm{C} 52 & -158.9(2) \\ \mathrm{N} 1-\mathrm{C} 11-\mathrm{C} 16-\mathrm{C} 15 & 179.5(3) & \mathrm{C} 56-\mathrm{C} 51-\mathrm{C} 52-\mathrm{C} 53 & 0.2(4) \\ \mathrm{C} 14-\mathrm{C} 15-\mathrm{C} 16-\mathrm{C} 11 & -0.9(5) & \mathrm{O} 51-\mathrm{C} 51-\mathrm{C} 52-\mathrm{C} 53 & -178.3(2) \\ \mathrm{C} 45-\mathrm{N} 41-\mathrm{N} 42-\mathrm{C} 422 & 174.3(2) & \mathrm{C} 56-\mathrm{C} 51-\mathrm{C} 52-\mathrm{C} 57 & -178.4(3) \\ \mathrm{C} 45-\mathrm{N} 41-\mathrm{N} 42-\mathrm{C} 43 & -4.4(3) & \mathrm{O} 51-\mathrm{C} 51-\mathrm{C} 52-\mathrm{C} 57 & 3.1(4) \\ \mathrm{C} 422-\mathrm{N} 42-\mathrm{C} 43-\mathrm{C} 4 & 64.4(3) & \mathrm{C} 51-\mathrm{C} 52-\mathrm{C} 53-\mathrm{C} 54 & -0.2(5) \\ \mathrm{N} 41-\mathrm{N} 42-\mathrm{C} 43-\mathrm{C} 4 & -117.0(2) & \mathrm{C} 57-\mathrm{C} 52-\mathrm{C} 53-\mathrm{C} 54 & 178.4(3) \\ \mathrm{C} 422-\mathrm{N} 42-\mathrm{C} 43-\mathrm{C} 44 & -170.8(2) & \mathrm{C} 52-\mathrm{C} 53-\mathrm{C} 54-\mathrm{C} 55 & -0.5(6) \\ \mathrm{N} 41-\mathrm{N} 42-\mathrm{C} 43-\mathrm{C} 44 & 7.7(3) & \mathrm{C} 53-\mathrm{C} 54-\mathrm{C} 55-\mathrm{C} 56 & 1.1(6) \\ \mathrm{C} 5-\mathrm{C} 4-\mathrm{C} 43-\mathrm{N} 42 & 41.7(4) & \mathrm{C} 52-\mathrm{C} 51-\mathrm{C} 56-\mathrm{C} 55 & 0.4(4) \\ \mathrm{C} 3-\mathrm{C} 4-\mathrm{C} 43-\mathrm{N} 42 & -132.8(3) & \mathrm{O} 51-\mathrm{C} 51-\mathrm{C} 56-\mathrm{C} 55 & 178.8(2) \\ \mathrm{C} 5-\mathrm{C} 4-\mathrm{C} 43-\mathrm{C} 44 & -74.0(4) & \mathrm{C} 54-\mathrm{C} 55-\mathrm{C} 56-\mathrm{C} 51 & -1.0(5) \\ \mathrm{C} 3-\mathrm{C} 4-\mathrm{C} 43-\mathrm{C} 44 & 111.5(3) & & \end{array}$

Hydrogen-bond geometry $\left(\AA,{ }^{\circ}\right)$

\begin{tabular}{lllll}
\hline$D-\mathrm{H} \cdots A$ & $D-\mathrm{H}$ & $\mathrm{H} \cdots A$ & $D \cdots A$ & $D-\mathrm{H} \cdots A$ \\
\hline $\mathrm{N} 423-\mathrm{H} 42 A \cdots \mathrm{N} 2^{\mathrm{i}}$ & $0.92(3)$ & $2.28(3)$ & $3.111(3)$ & $150(2)$ \\
$\mathrm{N} 423-\mathrm{H} 42 A \cdots \mathrm{N} 41$ & $0.92(3)$ & $2.27(3)$ & $2.628(4)$ & $103(2)$ \\
\hline
\end{tabular}

Symmetry code: (i) $x+1, y, z$.

5'-(2,4-Dichlorophenoxy)-3'-methyl-1'-phenyl-5-(thiophen-2-yl)-3,4-dihydro-1'H,2H-3,4'-bipyrazole-2carbothioamide hemihydrate (Ib)

Crystal data

$2 \mathrm{C}_{24} \mathrm{H}_{19} \mathrm{Cl}_{2} \mathrm{~N}_{5} \mathrm{OS}_{2} \cdot \mathrm{H}_{2} \mathrm{O}$

$M_{r}=1074.94$

Monoclinic, $P 2 / c$

$a=15.037$ (1) $\AA$

$b=8.4266(6) \AA$

$c=19.471(1) \AA$

$\beta=96.246(6)^{\circ}$

$V=2452.5(3) \AA^{3}$

$Z=2$

Data collection

Oxford Diffraction Xcalibur with Sapphire

CCD detector

diffractometer

Radiation source: Enhance (Mo) X-ray Source

Graphite monochromator

$\omega$ scans

Absorption correction: multi-scan

(CrysAlis RED; Oxford Diffraction, 2009)

$T_{\min }=0.922, T_{\max }=0.946$
$F(000)=1108$

$D_{\mathrm{x}}=1.456 \mathrm{Mg} \mathrm{m}^{-3}$

Mo $K \alpha$ radiation, $\lambda=0.71073 \AA$

Cell parameters from 5307 reflections

$\theta=2.6-27.9^{\circ}$

$\mu=0.47 \mathrm{~mm}^{-1}$

$T=296 \mathrm{~K}$

Needle, orange

$0.36 \times 0.12 \times 0.12 \mathrm{~mm}$

10494 measured reflections

5290 independent reflections

3075 reflections with $I>2 \sigma(I)$

$R_{\text {int }}=0.033$

$\theta_{\max }=27.6^{\circ}, \theta_{\min }=2.6^{\circ}$

$h=-19 \rightarrow 13$

$k=-10 \rightarrow 10$

$l=-25 \rightarrow 25$ 


\section{Refinement}

Refinement on $F^{2}$

Least-squares matrix: full

$R\left[F^{2}>2 \sigma\left(F^{2}\right)\right]=0.046$

$w R\left(F^{2}\right)=0.097$

$S=0.98$

5290 reflections

335 parameters

10 restraints
Primary atom site location: difference Fourier map

Hydrogen site location: mixed

$\mathrm{H}$ atoms treated by a mixture of independent and constrained refinement

$w=1 /\left[\sigma^{2}\left(F_{\mathrm{o}}^{2}\right)+(0.0411 P)^{2}\right]$ where $P=\left(F_{\mathrm{o}}^{2}+2 F_{\mathrm{c}}{ }^{2}\right) / 3$

$(\Delta / \sigma)_{\max }=0.001$

$\Delta \rho_{\max }=0.26 \mathrm{e} \AA^{-3}$

$\Delta \rho_{\min }=-0.30$ e $\AA^{-3}$

Special details

Experimental. CrysAlis RED, Oxford Diffraction Ltd., 2009 Empirical absorption correction using spherical harmonics, implemented in SCALE3 ABSPACK scaling algorithm.

Geometry. All esds (except the esd in the dihedral angle between two 1.s. planes) are estimated using the full covariance matrix. The cell esds are taken into account individually in the estimation of esds in distances, angles and torsion angles; correlations between esds in cell parameters are only used when they are defined by crystal symmetry. An approximate (isotropic) treatment of cell esds is used for estimating esds involving l.s. planes.

Fractional atomic coordinates and isotropic or equivalent isotropic displacement parameters $\left(\AA^{2}\right)$

\begin{tabular}{llllll}
\hline & $x$ & $y$ & $z$ & $U_{\text {iso }}^{*} / U_{\text {eq }}$ & Occ. $(<1)$ \\
\hline N1 & $0.68939(13)$ & $0.4363(2)$ & $0.37947(9)$ & $0.0343(5)$ & \\
N2 & $0.61354(13)$ & $0.3752(2)$ & $0.34400(9)$ & $0.0356(5)$ & \\
C3 & $0.59656(15)$ & $0.2419(3)$ & $0.37692(11)$ & $0.0318(5)$ & \\
C4 & $0.66061(15)$ & $0.2149(3)$ & $0.43422(11)$ & $0.0296(5)$ & \\
C5 & $0.71767(15)$ & $0.3404(3)$ & $0.43322(11)$ & $0.0308(5)$ & \\
C11 & $0.72930(16)$ & $0.5768(3)$ & $0.35564(11)$ & $0.0354(6)$ & $0.0602(8)$ \\
C12 & $0.81789(18)$ & $0.5775(3)$ & $0.34460(15)$ & $0.072^{*}$ \\
H12 & 0.8535 & 0.4889 & 0.3557 & $0.0748(10)$ \\
C13 & $0.8538(2)$ & $0.7111(4)$ & $0.31678(17)$ & $0.090^{*}$ \\
H13 & 0.9137 & 0.7127 & 0.3089 & $0.0580(8)$ \\
C14 & $0.8006(2)$ & $0.8415(3)$ & $0.30086(14)$ & $0.070^{*}$ \\
H14 & 0.8244 & 0.9307 & 0.2815 & $0.0471(7)$ \\
C15 & $0.7138(2)$ & $0.8410(3)$ & $0.31316(13)$ & $0.057^{*}$ \\
H15 & 0.6788 & 0.9306 & 0.3028 & $0.0384(6)$ \\
C16 & $0.67667(17)$ & $0.7089(3)$ & $0.34090(11)$ & $0.046^{*}$ \\
H16 & 0.6170 & 0.7092 & 0.3495 & $0.0438(6)$ \\
C31 & $0.51811(17)$ & $0.1418(3)$ & $0.35213(13)$ & $0.066^{*}$ \\
H31A & 0.4846 & 0.1180 & 0.3901 & $0.066^{*}$ \\
H31B & 0.5384 & 0.0447 & 0.3332 & $0.066^{*}$ \\
H31C & 0.4807 & 0.1979 & 0.3171 & $0.0355(5)$ \\
N41 & $0.75120(13)$ & $0.0972(2)$ & $0.59603(9)$ & $0.0344(5)$ \\
N42 & $0.67049(13)$ & $0.1362(2)$ & $0.55799(9)$ & $0.0335(6)$ \\
C43 & $0.66437(16)$ & $0.0806(3)$ & $0.48505(11)$ & $0.040^{*}$ \\
H43 & 0.6115 & 0.0131 & 0.4751 & $0.0404(6)$ \\
C44 & $0.74925(17)$ & $-0.0209(3)$ & $0.48690(12)$ & $0.048^{*}$ \\
H44A & 0.7346 & -0.1326 & 0.4813 & $0.048^{*}$ \\
H44B & 0.7855 & 0.0110 & 0.4510 &
\end{tabular}




\begin{tabular}{|c|c|c|c|c|c|}
\hline $\mathrm{C} 45$ & $0.79663(16)$ & 0.0117 & $0.55736(11)$ & $0.0336(6)$ & \\
\hline S421 & $0.50684(4)$ & $0.25555(8)$ & $0.54387(3)$ & $0.0477(2)$ & \\
\hline $\mathrm{C} 422$ & $0.60539(16)$ & 0.2113 & $0.58829(12)$ & $0.0361(6)$ & \\
\hline N423 & $0.62484(18)$ & $0.2475(3)$ & $0.65504(11)$ & $0.0512(7)$ & \\
\hline $\mathrm{H} 42 \mathrm{~A}$ & $0.585(2)$ & $0.293(3)$ & $0.6745(14)$ & $0.061 *$ & \\
\hline H42B & $0.675(2)$ & $0.220(3)$ & $0.6753(14)$ & $0.061 *$ & \\
\hline S451 & $0.93613(5)$ & $-0.02351(9)$ & $0.66256(4)$ & 0.0493 & $0.951(3)$ \\
\hline C452 & $0.88401(16)$ & -0.0510 & $0.58006(12)$ & $0.0365(6)$ & $0.951(3)$ \\
\hline $\mathrm{C} 453$ & $0.9356(3)$ & $-0.1417(6)$ & $0.5435(2)$ & $0.0475(9)$ & $0.951(3)$ \\
\hline H453 & 0.9188 & -0.1700 & 0.4977 & $0.057 *$ & $0.951(3)$ \\
\hline $\mathrm{C} 454$ & $1.0167(2)$ & $-0.1900(7)$ & $0.5800(2)$ & $0.0524(14)$ & $0.951(3)$ \\
\hline H454 & 1.0593 & -0.2517 & 0.5612 & $0.063 *$ & $0.951(3)$ \\
\hline $\mathrm{C} 455$ & $1.0251(2)$ & $-0.1363(4)$ & $0.64507(18)$ & $0.0537(12)$ & $0.951(3)$ \\
\hline H455 & 1.0740 & -0.1580 & 0.6772 & $0.064 *$ & $0.951(3)$ \\
\hline S551 & $0.937(2)$ & $-0.159(4)$ & $0.5215(11)$ & $0.0475(9)$ & 0.049 \\
\hline C552 & $0.88401(16)$ & -0.0510 & $0.58006(12)$ & $0.0365(6)$ & 0.049 (3) \\
\hline C553 & $0.944(2)$ & $-0.005(7)$ & $0.633(2)$ & 0.0493 & 0.049 \\
\hline H553 & 0.9341 & 0.0767 & 0.6631 & $0.059 *$ & 0.049 \\
\hline C554 & $1.024(3)$ & $-0.094(11)$ & $0.637(3)$ & $0.0537(12)$ & 0.049 (3) \\
\hline H554 & 1.0649 & -0.1006 & 0.6763 & $0.064 *$ & 0.049 (3) \\
\hline C555 & $1.032(4)$ & -0.169 (16) & $0.577(4)$ & $0.0524(14)$ & 0.049 (3) \\
\hline H555 & 1.0839 & -0.2211 & 0.5676 & $0.063 *$ & 0.049 (3) \\
\hline O51 & $0.79155(10)$ & $0.37875(17)$ & $0.47791(7)$ & 0.0338 (4) & \\
\hline C51 & $0.77558(16)$ & 0.4711 & $0.53498(11)$ & $0.0326(6)$ & \\
\hline C52 & $0.84468(16)$ & 0.4851 & $0.58742(12)$ & $0.0366(6)$ & \\
\hline $\mathrm{Cl} 52$ & $0.94533(5)$ & $0.39239(9)$ & $0.58023(4)$ & $0.0618(2)$ & \\
\hline $\mathrm{C} 53$ & 0.83348 (19) & $0.5733(3)$ & $0.64567(13)$ & $0.0464(7)$ & \\
\hline H53 & 0.8799 & 0.5827 & 0.6811 & $0.056^{*}$ & \\
\hline C54 & $0.7533(2)$ & $0.6467(3)$ & $0.65044(13)$ & $0.0453(7)$ & \\
\hline $\mathrm{Cl} 54$ & $0.73886(6)$ & $0.75872(9)$ & $0.72375(4)$ & 0.0733 & \\
\hline C55 & $0.68368(19)$ & 0.6329 & $0.59891(14)$ & $0.0496(7)$ & \\
\hline H55 & 0.6294 & 0.6826 & 0.6032 & $0.060 *$ & \\
\hline C56 & $0.69494(17)$ & $0.5446(3)$ & $0.54082(13)$ & $0.0424(6)$ & \\
\hline H56 & 0.6482 & 0.5348 & 0.5057 & $0.051^{*}$ & \\
\hline O61 & 0.5000 & $0.5746(3)$ & 0.2500 & $0.0605(9)$ & \\
\hline H61 & $0.532(2)$ & $0.511(3)$ & $0.2784(15)$ & $0.091 *$ & \\
\hline
\end{tabular}

Atomic displacement parameters $\left(\AA^{2}\right)$

\begin{tabular}{lllllll}
\hline & $U^{11}$ & $U^{22}$ & $U^{33}$ & $U^{12}$ & $U^{13}$ & $U^{23}$ \\
\hline $\mathrm{N} 1$ & $0.0301(11)$ & $0.0402(12)$ & $0.0312(11)$ & $-0.0022(9)$ & $-0.0028(9)$ & $0.0066(9)$ \\
$\mathrm{N} 2$ & $0.0304(12)$ & $0.0441(12)$ & $0.0306(11)$ & $-0.0018(10)$ & $-0.0039(9)$ & $0.0035(9)$ \\
C3 & $0.0300(13)$ & $0.0390(14)$ & $0.0263(12)$ & $0.0011(11)$ & $0.0029(10)$ & $-0.0019(11)$ \\
C4 & $0.0306(13)$ & $0.0325(13)$ & $0.0254(12)$ & $0.0022(11)$ & $0.0013(10)$ & $0.0006(10)$ \\
C5 & $0.0259(13)$ & $0.0372(14)$ & $0.0280(12)$ & $0.0040(11)$ & $-0.0024(10)$ & $-0.0010(11)$ \\
C11 & $0.0334(14)$ & $0.0411(15)$ & $0.0309(13)$ & $-0.0047(12)$ & $0.0001(11)$ & $0.0056(11)$ \\
C12 & $0.0372(17)$ & $0.0615(19)$ & $0.082(2)$ & $0.0038(15)$ & $0.0074(16)$ & $0.0298(17)$ \\
C13 & $0.0392(18)$ & $0.087(2)$ & $0.098(3)$ & $-0.0103(18)$ & $0.0100(18)$ & $0.039(2)$
\end{tabular}




\begin{tabular}{|c|c|c|c|c|c|c|}
\hline $\mathrm{C} 14$ & $0.058(2)$ & $0.0583(19)$ & $0.0562(18)$ & $-0.0216(17)$ & $-0.0028(16)$ & $0.0207(15)$ \\
\hline $\mathrm{C} 15$ & $0.0563(19)$ & $0.0407(16)$ & $0.0432(15)$ & $-0.0018(14)$ & $0.0001(14)$ & $0.0049(13)$ \\
\hline $\mathrm{C} 16$ & $0.0383(15)$ & $0.0438(15)$ & $0.0332(14)$ & $-0.0012(12)$ & $0.0046(12)$ & $-0.0012(12)$ \\
\hline $\mathrm{C} 31$ & $0.0385(15)$ & $0.0491(16)$ & $0.0421(15)$ & $-0.0018(13)$ & $-0.0030(12)$ & $-0.0019(12)$ \\
\hline N41 & $0.0348(12)$ & $0.0398(12)$ & $0.0314(11)$ & $0.0032(10)$ & $0.0015(9)$ & $0.0036(9)$ \\
\hline $\mathrm{N} 42$ & $0.0341(12)$ & $0.0424(12)$ & $0.0264(10)$ & $0.0080(10)$ & $0.0017(9)$ & $0.0004(9)$ \\
\hline $\mathrm{C} 43$ & $0.0367(14)$ & $0.0345(13)$ & $0.0286(12)$ & $-0.0024(11)$ & $0.0009(11)$ & $-0.0001(11)$ \\
\hline $\mathrm{C} 44$ & $0.0496(16)$ & $0.0359(14)$ & $0.0348(13)$ & $0.0073(13)$ & $0.0010(12)$ & $-0.0001(11)$ \\
\hline $\mathrm{C} 45$ & $0.0412(15)$ & $0.0284(13)$ & $0.0313(13)$ & $0.0013(12)$ & $0.0047(12)$ & $0.0032(11)$ \\
\hline S421 & $0.0361(4)$ & $0.0614(5)$ & $0.0457(4)$ & $0.0054(3)$ & $0.0045(3)$ & $0.0036(3)$ \\
\hline $\mathrm{C} 422$ & $0.0382(15)$ & $0.0369(14)$ & $0.0336(13)$ & $-0.0021(12)$ & $0.0062(12)$ & $0.0045(11)$ \\
\hline $\mathrm{N} 423$ & $0.0508(16)$ & $0.0714(17)$ & $0.0317(13)$ & $0.0165(13)$ & $0.0051(11)$ & $-0.0039(12)$ \\
\hline S451 & $0.0460(5)$ & $0.0588(5)$ & $0.0412(5)$ & $0.0101(4)$ & -0.0039 (4) & $-0.0069(4)$ \\
\hline $\mathrm{C} 452$ & $0.0382(15)$ & $0.0347(14)$ & $0.0361(13)$ & $0.0032(12)$ & $0.0026(12)$ & $0.0038(11)$ \\
\hline $\mathrm{C} 453$ & $0.0523(18)$ & $0.057(2)$ & $0.035(3)$ & $0.0135(15)$ & $0.009(2)$ & $0.000(2)$ \\
\hline $\mathrm{C} 454$ & $0.041(2)$ & $0.058(3)$ & $0.0590(19)$ & $0.013(2)$ & $0.0074(17)$ & $-0.0020(17)$ \\
\hline $\mathrm{C} 455$ & $0.0405(17)$ & $0.056(3)$ & $0.061(2)$ & $0.0099(16)$ & $-0.0100(15)$ & $0.0011(18)$ \\
\hline S551 & $0.0523(18)$ & $0.057(2)$ & $0.035(3)$ & $0.0135(15)$ & $0.009(2)$ & $0.000(2)$ \\
\hline C552 & $0.0382(15)$ & $0.0347(14)$ & $0.0361(13)$ & $0.0032(12)$ & $0.0026(12)$ & $0.0038(11)$ \\
\hline C553 & $0.0460(5)$ & $0.0588(5)$ & $0.0412(5)$ & $0.0101(4)$ & $-0.0039(4)$ & $-0.0069(4)$ \\
\hline C554 & $0.0405(17)$ & $0.056(3)$ & $0.061(2)$ & $0.0099(16)$ & $-0.0100(15)$ & $0.0011(18)$ \\
\hline C555 & $0.041(2)$ & $0.058(3)$ & $0.0590(19)$ & $0.013(2)$ & $0.0074(17)$ & $-0.0020(17)$ \\
\hline O51 & $0.0261(9)$ & $0.0424(10)$ & $0.0317(9)$ & $0.0033(7)$ & $-0.0025(7)$ & $-0.0042(8)$ \\
\hline C51 & $0.0356(14)$ & $0.0287(13)$ & $0.0333(13)$ & $-0.0006(11)$ & $0.0031(11)$ & $0.0024(11)$ \\
\hline C52 & $0.0320(14)$ & $0.0378(14)$ & $0.0392(14)$ & $0.0002(11)$ & $-0.0004(12)$ & $0.0004(12)$ \\
\hline $\mathrm{Cl} 52$ & $0.0389(4)$ & $0.0757(5)$ & $0.0665(5)$ & $0.0146(4)$ & $-0.0133(4)$ & $-0.0190(4)$ \\
\hline $\mathrm{C} 53$ & $0.0496(18)$ & $0.0501(17)$ & $0.0385(15)$ & $-0.0095(14)$ & $0.0007(13)$ & $-0.0065(13)$ \\
\hline C54 & $0.0579(19)$ & $0.0391(15)$ & $0.0411(15)$ & $-0.0095(14)$ & $0.0155(14)$ & $-0.0061(12)$ \\
\hline $\mathrm{Cl} 54$ & $0.0881(6)$ & $0.0778(6)$ & 0.0589 & $-0.0104(5)$ & 0.0293 & $-0.0278(4)$ \\
\hline C55 & $0.0437(17)$ & $0.0510(17)$ & $0.0564(18)$ & $0.0062(13)$ & $0.0157(15)$ & $-0.0078(14)$ \\
\hline C56 & $0.0352(15)$ & $0.0455(16)$ & $0.0454(15)$ & $0.0029(12)$ & $-0.0004(13)$ & $-0.0032(13)$ \\
\hline O61 & $0.064(2)$ & $0.0498(18)$ & $0.0594(19)$ & 0.000 & $-0.0293(15)$ & 0.000 \\
\hline
\end{tabular}

Geometric parameters $\left(\AA,{ }^{\circ}\right)$

\begin{tabular}{llll}
\hline $\mathrm{N} 1-\mathrm{C} 5$ & $1.354(3)$ & $\mathrm{C} 45-\mathrm{C} 452$ & $1.440(3)$ \\
$\mathrm{N} 1-\mathrm{N} 2$ & $1.368(2)$ & $\mathrm{S} 421-\mathrm{C} 422$ & $1.675(2)$ \\
$\mathrm{N} 1-\mathrm{C} 11$ & $1.427(3)$ & $\mathrm{C} 422-\mathrm{N} 423$ & $1.336(3)$ \\
$\mathrm{N} 2-\mathrm{C} 3$ & $1.332(3)$ & $\mathrm{N} 423-\mathrm{H} 42 \mathrm{~A}$ & $0.83(3)$ \\
$\mathrm{C} 3-\mathrm{C} 4$ & $1.411(3)$ & $\mathrm{N} 423-\mathrm{H} 42 \mathrm{~B}$ & $0.84(3)$ \\
$\mathrm{C} 3-\mathrm{C} 31$ & $1.488(3)$ & $\mathrm{S} 451-\mathrm{C} 455$ & $1.705(3)$ \\
$\mathrm{C} 4-\mathrm{C} 5$ & $1.363(3)$ & $\mathrm{S} 451-\mathrm{C} 452$ & $1.725(2)$ \\
$\mathrm{C} 4-\mathrm{C} 43$ & $1.500(3)$ & $\mathrm{C} 452-\mathrm{C} 453$ & $1.347(5)$ \\
$\mathrm{C} 5-\mathrm{O} 51$ & $1.373(2)$ & $\mathrm{C} 453-\mathrm{C} 454$ & $1.404(5)$ \\
$\mathrm{C} 11-\mathrm{C} 12$ & $1.372(3)$ & $\mathrm{C} 453-\mathrm{H} 453$ & 0.9300 \\
$\mathrm{C} 11-\mathrm{C} 16$ & $1.378(3)$ & $\mathrm{C} 454-\mathrm{C} 455$ & $1.339(4)$ \\
$\mathrm{C} 12-\mathrm{C} 13$ & $1.385(4)$ & $\mathrm{C} 454-\mathrm{H} 454$ & 0.9300 \\
$\mathrm{C} 12-\mathrm{H} 12$ & 0.9300 & $\mathrm{C} 455-\mathrm{H} 455$ & 0.9300
\end{tabular}




\begin{tabular}{|c|c|c|c|}
\hline $\mathrm{C} 13-\mathrm{C} 14$ & $1.375(4)$ & $\mathrm{S} 551-\mathrm{C} 555$ & $1.707(11)$ \\
\hline $\mathrm{C} 13-\mathrm{H} 13$ & 0.9300 & C553-C554 & $1.407(11)$ \\
\hline $\mathrm{C} 14-\mathrm{C} 15$ & $1.352(4)$ & C553-H553 & 0.9300 \\
\hline C14-H14 & 0.9300 & C554-C555 & $1.342(10)$ \\
\hline $\mathrm{C} 15-\mathrm{C} 16$ & $1.382(3)$ & C554-H554 & 0.9300 \\
\hline $\mathrm{C} 15-\mathrm{H} 15$ & 0.9300 & C555-H555 & 0.9300 \\
\hline $\mathrm{C} 16-\mathrm{H} 16$ & 0.9300 & $\mathrm{O} 51-\mathrm{C} 51$ & $1.399(3)$ \\
\hline $\mathrm{C} 31-\mathrm{H} 31 \mathrm{~A}$ & 0.9600 & $\mathrm{C} 51-\mathrm{C} 56$ & $1.377(3)$ \\
\hline $\mathrm{C} 31-\mathrm{H} 31 \mathrm{~B}$ & 0.9600 & $\mathrm{C} 51-\mathrm{C} 52$ & $1.380(3)$ \\
\hline $\mathrm{C} 31-\mathrm{H} 31 \mathrm{C}$ & 0.9600 & $\mathrm{C} 52-\mathrm{C} 53$ & $1.382(3)$ \\
\hline $\mathrm{N} 41-\mathrm{C} 45$ & $1.290(3)$ & $\mathrm{C} 52-\mathrm{Cl} 52$ & $1.722(2)$ \\
\hline $\mathrm{N} 41-\mathrm{N} 42$ & $1.391(2)$ & $\mathrm{C} 53-\mathrm{C} 54$ & $1.366(4)$ \\
\hline $\mathrm{N} 42-\mathrm{C} 422$ & $1.354(3)$ & C53-H53 & 0.9300 \\
\hline $\mathrm{N} 42-\mathrm{C} 43$ & $1.489(3)$ & $\mathrm{C} 54-\mathrm{C} 55$ & $1.374(4)$ \\
\hline $\mathrm{C} 43-\mathrm{C} 44$ & $1.534(3)$ & $\mathrm{C} 54-\mathrm{Cl} 54$ & $1.745(3)$ \\
\hline $\mathrm{C} 43-\mathrm{H} 43$ & 0.9800 & $\mathrm{C} 55-\mathrm{C} 56$ & $1.379(3)$ \\
\hline $\mathrm{C} 44-\mathrm{C} 45$ & $1.501(3)$ & C55-H55 & 0.9300 \\
\hline $\mathrm{C} 44-\mathrm{H} 44 \mathrm{~A}$ & 0.9700 & C56-H56 & 0.9300 \\
\hline C44-H44B & 0.9700 & O61-H61 & $0.88(3)$ \\
\hline $\mathrm{C} 5-\mathrm{N} 1-\mathrm{N} 2$ & $109.89(18)$ & $\mathrm{C} 43-\mathrm{C} 44-\mathrm{H} 44 \mathrm{~B}$ & 111.1 \\
\hline $\mathrm{C} 5-\mathrm{N} 1-\mathrm{C} 11$ & $129.69(19)$ & $\mathrm{H} 44 \mathrm{~A}-\mathrm{C} 44-\mathrm{H} 44 \mathrm{~B}$ & 109.1 \\
\hline $\mathrm{N} 2-\mathrm{N} 1-\mathrm{C} 11$ & $120.32(17)$ & $\mathrm{N} 41-\mathrm{C} 45-\mathrm{C} 452$ & $123.3(2)$ \\
\hline $\mathrm{C} 3-\mathrm{N} 2-\mathrm{N} 1$ & $105.50(17)$ & $\mathrm{N} 41-\mathrm{C} 45-\mathrm{C} 44$ & $114.0(2)$ \\
\hline $\mathrm{N} 2-\mathrm{C} 3-\mathrm{C} 4$ & $111.5(2)$ & $\mathrm{C} 452-\mathrm{C} 45-\mathrm{C} 44$ & $122.7(2)$ \\
\hline $\mathrm{N} 2-\mathrm{C} 3-\mathrm{C} 31$ & $120.6(2)$ & $\mathrm{N} 423-\mathrm{C} 422-\mathrm{N} 42$ & $116.0(2)$ \\
\hline $\mathrm{C} 4-\mathrm{C} 3-\mathrm{C} 31$ & $127.9(2)$ & $\mathrm{N} 423-\mathrm{C} 422-\mathrm{S} 421$ & $122.6(2)$ \\
\hline $\mathrm{C} 5-\mathrm{C} 4-\mathrm{C} 3$ & $103.90(19)$ & $\mathrm{N} 42-\mathrm{C} 422-\mathrm{S} 421$ & $121.36(18)$ \\
\hline $\mathrm{C} 5-\mathrm{C} 4-\mathrm{C} 43$ & $128.0(2)$ & $\mathrm{C} 422-\mathrm{N} 423-\mathrm{H} 42 \mathrm{~A}$ & $117.2(19)$ \\
\hline $\mathrm{C} 3-\mathrm{C} 4-\mathrm{C} 43$ & $128.1(2)$ & $\mathrm{C} 422-\mathrm{N} 423-\mathrm{H} 42 \mathrm{~B}$ & $118.9(19)$ \\
\hline $\mathrm{N} 1-\mathrm{C} 5-\mathrm{C} 4$ & $109.20(19)$ & $\mathrm{H} 42 \mathrm{~A}-\mathrm{N} 423-\mathrm{H} 42 \mathrm{~B}$ & $124(3)$ \\
\hline $\mathrm{N} 1-\mathrm{C} 5-\mathrm{O} 51$ & $120.9(2)$ & $\mathrm{C} 455-\mathrm{S} 451-\mathrm{C} 452$ & $91.49(14)$ \\
\hline $\mathrm{C} 4-\mathrm{C} 5-\mathrm{O} 51$ & $129.8(2)$ & $\mathrm{C} 453-\mathrm{C} 452-\mathrm{C} 45$ & $127.2(3)$ \\
\hline $\mathrm{C} 12-\mathrm{C} 11-\mathrm{C} 16$ & $120.6(2)$ & $\mathrm{C} 453-\mathrm{C} 452-\mathrm{S} 451$ & $109.8(2)$ \\
\hline $\mathrm{C} 12-\mathrm{C} 11-\mathrm{N} 1$ & $120.3(2)$ & $\mathrm{C} 45-\mathrm{C} 452-\mathrm{S} 451$ & $122.94(19)$ \\
\hline $\mathrm{C} 16-\mathrm{C} 11-\mathrm{N} 1$ & $119.1(2)$ & $\mathrm{C} 452-\mathrm{C} 453-\mathrm{C} 454$ & $114.6(3)$ \\
\hline $\mathrm{C} 11-\mathrm{C} 12-\mathrm{C} 13$ & $119.5(3)$ & $\mathrm{C} 452-\mathrm{C} 453-\mathrm{H} 453$ & 122.7 \\
\hline $\mathrm{C} 11-\mathrm{C} 12-\mathrm{H} 12$ & 120.3 & $\mathrm{C} 454-\mathrm{C} 453-\mathrm{H} 453$ & 122.7 \\
\hline $\mathrm{C} 13-\mathrm{C} 12-\mathrm{H} 12$ & 120.3 & $\mathrm{C} 455-\mathrm{C} 454-\mathrm{C} 453$ & $111.6(3)$ \\
\hline $\mathrm{C} 14-\mathrm{C} 13-\mathrm{C} 12$ & $119.7(3)$ & $\mathrm{C} 455-\mathrm{C} 454-\mathrm{H} 454$ & 124.2 \\
\hline $\mathrm{C} 14-\mathrm{C} 13-\mathrm{H} 13$ & 120.1 & $\mathrm{C} 453-\mathrm{C} 454-\mathrm{H} 454$ & 124.2 \\
\hline $\mathrm{C} 12-\mathrm{C} 13-\mathrm{H} 13$ & 120.1 & $\mathrm{C} 454-\mathrm{C} 455-\mathrm{S} 451$ & $112.5(2)$ \\
\hline $\mathrm{C} 15-\mathrm{C} 14-\mathrm{C} 13$ & $120.5(3)$ & $\mathrm{C} 454-\mathrm{C} 455-\mathrm{H} 455$ & 123.7 \\
\hline $\mathrm{C} 15-\mathrm{C} 14-\mathrm{H} 14$ & 119.8 & $\mathrm{~S} 451-\mathrm{C} 455-\mathrm{H} 455$ & 123.7 \\
\hline $\mathrm{C} 13-\mathrm{C} 14-\mathrm{H} 14$ & 119.8 & $\mathrm{C} 554-\mathrm{C} 553-\mathrm{H} 553$ & 123.6 \\
\hline $\mathrm{C} 14-\mathrm{C} 15-\mathrm{C} 16$ & $120.7(3)$ & $\mathrm{C} 555-\mathrm{C} 554-\mathrm{C} 553$ & $111.1(15)$ \\
\hline $\mathrm{C} 14-\mathrm{C} 15-\mathrm{H} 15$ & 119.6 & C555-C554-H554 & 124.5 \\
\hline $\mathrm{C} 16-\mathrm{C} 15-\mathrm{H} 15$ & 119.6 & C553-C554-H554 & 124.5 \\
\hline
\end{tabular}




\begin{tabular}{|c|c|c|c|}
\hline $\mathrm{C} 11-\mathrm{C} 16-\mathrm{C} 15$ & $119.0(2)$ & $\mathrm{C} 554-\mathrm{C} 555-\mathrm{S} 551$ & $112.1(11)$ \\
\hline $\mathrm{C} 11-\mathrm{C} 16-\mathrm{H} 16$ & 120.5 & $\mathrm{C} 554-\mathrm{C} 555-\mathrm{H} 555$ & 123.9 \\
\hline $\mathrm{C} 15-\mathrm{C} 16-\mathrm{H} 16$ & 120.5 & S551-C555-H555 & 123.9 \\
\hline $\mathrm{C} 3-\mathrm{C} 31-\mathrm{H} 31 \mathrm{~A}$ & 109.5 & $\mathrm{C} 5-\mathrm{O} 51-\mathrm{C} 51$ & $115.94(17)$ \\
\hline $\mathrm{C} 3-\mathrm{C} 31-\mathrm{H} 31 \mathrm{~B}$ & 109.5 & $\mathrm{C} 56-\mathrm{C} 51-\mathrm{C} 52$ & $120.0(2)$ \\
\hline $\mathrm{H} 31 \mathrm{~A}-\mathrm{C} 31-\mathrm{H} 31 \mathrm{~B}$ & 109.5 & $\mathrm{C} 56-\mathrm{C} 51-\mathrm{O} 51$ & $123.0(2)$ \\
\hline $\mathrm{C} 3-\mathrm{C} 31-\mathrm{H} 31 \mathrm{C}$ & 109.5 & $\mathrm{C} 52-\mathrm{C} 51-\mathrm{O} 51$ & $117.0(2)$ \\
\hline $\mathrm{H} 31 \mathrm{~A}-\mathrm{C} 31-\mathrm{H} 31 \mathrm{C}$ & 109.5 & $\mathrm{C} 51-\mathrm{C} 52-\mathrm{C} 53$ & $120.2(2)$ \\
\hline $\mathrm{H} 31 \mathrm{~B}-\mathrm{C} 31-\mathrm{H} 31 \mathrm{C}$ & 109.5 & $\mathrm{C} 51-\mathrm{C} 52-\mathrm{Cl} 52$ & $119.92(18)$ \\
\hline $\mathrm{C} 45-\mathrm{N} 41-\mathrm{N} 42$ & $107.92(18)$ & $\mathrm{C} 53-\mathrm{C} 52-\mathrm{Cl} 52$ & $119.83(19)$ \\
\hline $\mathrm{C} 422-\mathrm{N} 42-\mathrm{N} 41$ & $120.58(18)$ & $\mathrm{C} 54-\mathrm{C} 53-\mathrm{C} 52$ & $119.1(2)$ \\
\hline $\mathrm{C} 422-\mathrm{N} 42-\mathrm{C} 43$ & $126.17(19)$ & $\mathrm{C} 54-\mathrm{C} 53-\mathrm{H} 53$ & 120.4 \\
\hline $\mathrm{N} 41-\mathrm{N} 42-\mathrm{C} 43$ & $113.19(18)$ & $\mathrm{C} 52-\mathrm{C} 53-\mathrm{H} 53$ & 120.4 \\
\hline $\mathrm{N} 42-\mathrm{C} 43-\mathrm{C} 4$ & $112.72(18)$ & $\mathrm{C} 53-\mathrm{C} 54-\mathrm{C} 55$ & $121.3(2)$ \\
\hline $\mathrm{N} 42-\mathrm{C} 43-\mathrm{C} 44$ & $100.84(17)$ & $\mathrm{C} 53-\mathrm{C} 54-\mathrm{Cl} 54$ & $119.3(2)$ \\
\hline $\mathrm{C} 4-\mathrm{C} 43-\mathrm{C} 44$ & $114.06(19)$ & $\mathrm{C} 55-\mathrm{C} 54-\mathrm{Cl} 54$ & $119.3(2)$ \\
\hline $\mathrm{N} 42-\mathrm{C} 43-\mathrm{H} 43$ & 109.6 & $\mathrm{C} 54-\mathrm{C} 55-\mathrm{C} 56$ & $119.5(2)$ \\
\hline $\mathrm{C} 4-\mathrm{C} 43-\mathrm{H} 43$ & 109.6 & $\mathrm{C} 54-\mathrm{C} 55-\mathrm{H} 55$ & 120.2 \\
\hline $\mathrm{C} 44-\mathrm{C} 43-\mathrm{H} 43$ & 109.6 & $\mathrm{C} 56-\mathrm{C} 55-\mathrm{H} 55$ & 120.2 \\
\hline $\mathrm{C} 45-\mathrm{C} 44-\mathrm{C} 43$ & $103.32(18)$ & $\mathrm{C} 51-\mathrm{C} 56-\mathrm{C} 55$ & $119.9(2)$ \\
\hline $\mathrm{C} 45-\mathrm{C} 44-\mathrm{H} 44 \mathrm{~A}$ & 111.1 & $\mathrm{C} 51-\mathrm{C} 56-\mathrm{H} 56$ & 120.1 \\
\hline $\mathrm{C} 43-\mathrm{C} 44-\mathrm{H} 44 \mathrm{~A}$ & 111.1 & $\mathrm{C} 55-\mathrm{C} 56-\mathrm{H} 56$ & 120.1 \\
\hline $\mathrm{C} 45-\mathrm{C} 44-\mathrm{H} 44 \mathrm{~B}$ & 111.1 & & \\
\hline $\mathrm{C} 5-\mathrm{N} 1-\mathrm{N} 2-\mathrm{C} 3$ & $0.0(2)$ & $\mathrm{C} 4-\mathrm{C} 43-\mathrm{C} 44-\mathrm{C} 45$ & $113.3(2)$ \\
\hline $\mathrm{C} 11-\mathrm{N} 1-\mathrm{N} 2-\mathrm{C} 3$ & $176.76(19)$ & $\mathrm{N} 42-\mathrm{N} 41-\mathrm{C} 45-\mathrm{C} 452$ & $-179.7(2)$ \\
\hline $\mathrm{N} 1-\mathrm{N} 2-\mathrm{C} 3-\mathrm{C} 4$ & $0.3(2)$ & $\mathrm{N} 42-\mathrm{N} 41-\mathrm{C} 45-\mathrm{C} 44$ & $-1.3(3)$ \\
\hline $\mathrm{N} 1-\mathrm{N} 2-\mathrm{C} 3-\mathrm{C} 31$ & $-179.6(2)$ & $\mathrm{C} 43-\mathrm{C} 44-\mathrm{C} 45-\mathrm{N} 41$ & $6.2(3)$ \\
\hline $\mathrm{N} 2-\mathrm{C} 3-\mathrm{C} 4-\mathrm{C} 5$ & $-0.5(2)$ & $\mathrm{C} 43-\mathrm{C} 44-\mathrm{C} 45-\mathrm{C} 452$ & $-175.4(2)$ \\
\hline $\mathrm{C} 31-\mathrm{C} 3-\mathrm{C} 4-\mathrm{C} 5$ & $179.4(2)$ & $\mathrm{N} 41-\mathrm{N} 42-\mathrm{C} 422-\mathrm{N} 423$ & $1.5(3)$ \\
\hline $\mathrm{N} 2-\mathrm{C} 3-\mathrm{C} 4-\mathrm{C} 43$ & $178.8(2)$ & $\mathrm{C} 43-\mathrm{N} 42-\mathrm{C} 422-\mathrm{N} 423$ & $178.6(2)$ \\
\hline $\mathrm{C} 31-\mathrm{C} 3-\mathrm{C} 4-\mathrm{C} 43$ & $-1.4(4)$ & $\mathrm{N} 41-\mathrm{N} 42-\mathrm{C} 422-\mathrm{S} 421$ & $-178.60(16)$ \\
\hline $\mathrm{N} 2-\mathrm{N} 1-\mathrm{C} 5-\mathrm{C} 4$ & $-0.3(3)$ & $\mathrm{C} 43-\mathrm{N} 42-\mathrm{C} 422-\mathrm{S} 421$ & $-1.5(3)$ \\
\hline $\mathrm{C} 11-\mathrm{N} 1-\mathrm{C} 5-\mathrm{C} 4$ & $-176.7(2)$ & $\mathrm{N} 41-\mathrm{C} 45-\mathrm{C} 452-\mathrm{C} 453$ & $179.5(4)$ \\
\hline $\mathrm{N} 2-\mathrm{N} 1-\mathrm{C} 5-\mathrm{O} 51$ & $-178.18(18)$ & $\mathrm{C} 44-\mathrm{C} 45-\mathrm{C} 452-\mathrm{C} 453$ & $1.3(5)$ \\
\hline $\mathrm{C} 11-\mathrm{N} 1-\mathrm{C} 5-\mathrm{O} 51$ & $5.4(3)$ & $\mathrm{N} 41-\mathrm{C} 45-\mathrm{C} 452-\mathrm{S} 451$ & $2.1(3)$ \\
\hline $\mathrm{C} 3-\mathrm{C} 4-\mathrm{C} 5-\mathrm{N} 1$ & $0.4(2)$ & $\mathrm{C} 44-\mathrm{C} 45-\mathrm{C} 452-\mathrm{S} 451$ & $-176.17(18)$ \\
\hline $\mathrm{C} 43-\mathrm{C} 4-\mathrm{C} 5-\mathrm{N} 1$ & $-178.8(2)$ & $\mathrm{C} 455-\mathrm{S} 451-\mathrm{C} 452-\mathrm{C} 453$ & $-0.4(3)$ \\
\hline $\mathrm{C} 3-\mathrm{C} 4-\mathrm{C} 5-\mathrm{O} 51$ & $178.1(2)$ & $\mathrm{C} 455-\mathrm{S} 451-\mathrm{C} 452-\mathrm{C} 45$ & $177.4(2)$ \\
\hline $\mathrm{C} 43-\mathrm{C} 4-\mathrm{C} 5-\mathrm{O} 51$ & $-1.1(4)$ & $\mathrm{C} 45-\mathrm{C} 452-\mathrm{C} 453-\mathrm{C} 454$ & $-177.9(4)$ \\
\hline $\mathrm{C} 5-\mathrm{N} 1-\mathrm{C} 11-\mathrm{C} 12$ & $50.0(3)$ & $\mathrm{S} 451-\mathrm{C} 452-\mathrm{C} 453-\mathrm{C} 454$ & $-0.2(6)$ \\
\hline $\mathrm{N} 2-\mathrm{N} 1-\mathrm{C} 11-\mathrm{C} 12$ & $-126.0(3)$ & $\mathrm{C} 452-\mathrm{C} 453-\mathrm{C} 454-\mathrm{C} 455$ & $0.9(7)$ \\
\hline $\mathrm{C} 5-\mathrm{N} 1-\mathrm{C} 11-\mathrm{C} 16$ & $-132.9(2)$ & $\mathrm{C} 453-\mathrm{C} 454-\mathrm{C} 455-\mathrm{S} 451$ & $-1.2(6)$ \\
\hline $\mathrm{N} 2-\mathrm{N} 1-\mathrm{C} 11-\mathrm{C} 16$ & $51.0(3)$ & $\mathrm{C} 452-\mathrm{S} 451-\mathrm{C} 455-\mathrm{C} 454$ & $0.9(4)$ \\
\hline $\mathrm{C} 16-\mathrm{C} 11-\mathrm{C} 12-\mathrm{C} 13$ & $-1.6(4)$ & $\mathrm{C} 553-\mathrm{C} 554-\mathrm{C} 555-\mathrm{S} 551$ & $-10(12)$ \\
\hline $\mathrm{N} 1-\mathrm{C} 11-\mathrm{C} 12-\mathrm{C} 13$ & $175.4(2)$ & $\mathrm{N} 1-\mathrm{C} 5-\mathrm{O} 51-\mathrm{C} 51$ & $89.3(2)$ \\
\hline $\mathrm{C} 11-\mathrm{C} 12-\mathrm{C} 13-\mathrm{C} 14$ & $0.3(5)$ & $\mathrm{C} 4-\mathrm{C} 5-\mathrm{O} 51-\mathrm{C} 51$ & $-88.1(3)$ \\
\hline $\mathrm{C} 12-\mathrm{C} 13-\mathrm{C} 14-\mathrm{C} 15$ & $1.0(5)$ & $\mathrm{C} 5-\mathrm{O} 51-\mathrm{C} 51-\mathrm{C} 56$ & $-11.5(3)$ \\
\hline
\end{tabular}




$\begin{array}{llll}\mathrm{C} 13-\mathrm{C} 14-\mathrm{C} 15-\mathrm{C} 16 & -1.0(4) & \mathrm{C} 5-\mathrm{O} 51-\mathrm{C} 51-\mathrm{C} 52 & 167.5(2) \\ \mathrm{C} 12-\mathrm{C} 11-\mathrm{C} 16-\mathrm{C} 15 & 1.6(4) & \mathrm{C} 56-\mathrm{C} 51-\mathrm{C} 52-\mathrm{C} 53 & -0.4(3) \\ \mathrm{N} 1-\mathrm{C} 11-\mathrm{C} 16-\mathrm{C} 15 & -175.40(19) & \mathrm{O} 51-\mathrm{C} 51-\mathrm{C} 52-\mathrm{C} 53 & -179.4(2) \\ \mathrm{C} 14-\mathrm{C} 15-\mathrm{C} 16-\mathrm{C} 11 & -0.3(4) & \mathrm{C} 56-\mathrm{C} 51-\mathrm{C} 52-\mathrm{C} 152 & 179.72(18) \\ \mathrm{C} 45-\mathrm{N} 41-\mathrm{N} 42-\mathrm{C} 422 & 172.8(2) & \mathrm{O} 51-\mathrm{C} 51-\mathrm{C} 52-\mathrm{C} 152 & 0.7(3) \\ \mathrm{C} 45-\mathrm{N} 41-\mathrm{N} 42-\mathrm{C} 43 & -4.6(2) & \mathrm{C} 51-\mathrm{C} 52-\mathrm{C} 53-\mathrm{C} 54 & -0.2(4) \\ \mathrm{C} 422-\mathrm{N} 42-\mathrm{C} 43-\mathrm{C} 4 & 68.7(3) & \mathrm{C} 152-\mathrm{C} 52-\mathrm{C} 53-\mathrm{C} 54 & 179.7(2) \\ \mathrm{N} 41-\mathrm{N} 42-\mathrm{C} 43-\mathrm{C} 4 & -114.0(2) & \mathrm{C} 52-\mathrm{C} 53-\mathrm{C} 54-\mathrm{C} 55 & 0.7(4) \\ \mathrm{C} 422-\mathrm{N} 42-\mathrm{C} 43-\mathrm{C} 44 & -169.3(2) & \mathrm{C} 52-\mathrm{C} 53-\mathrm{C} 54-\mathrm{C} 154 & -179.85(18) \\ \mathrm{N} 41-\mathrm{N} 42-\mathrm{C} 43-\mathrm{C} 44 & 8.0(2) & \mathrm{C} 53-\mathrm{C} 54-\mathrm{C} 55-\mathrm{C} 56 & -0.7(4) \\ \mathrm{C} 5-\mathrm{C} 4-\mathrm{C} 43-\mathrm{N} 42 & 52.6(3) & \mathrm{C} 154-\mathrm{C} 54-\mathrm{C} 55-\mathrm{C} 56 & 179.89(19) \\ \mathrm{C} 3-\mathrm{C} 4-\mathrm{C} 43-\mathrm{N} 42 & -126.5(2) & \mathrm{C} 52-\mathrm{C} 51-\mathrm{C} 56-\mathrm{C} 55 & 0.4(4) \\ \mathrm{C} 5-\mathrm{C} 4-\mathrm{C} 43-\mathrm{C} 44 & -61.7(3) & \mathrm{O} 51-\mathrm{C} 51-\mathrm{C} 56-\mathrm{C} 55 & 179.4(2) \\ \mathrm{C} 3-\mathrm{C} 4-\mathrm{C} 43-\mathrm{C} 44 & 119.3(3) & \mathrm{C} 54-\mathrm{C} 55-\mathrm{C} 56-\mathrm{C} 51 & 0.1(4) \\ \mathrm{N} 42-\mathrm{C} 43-\mathrm{C} 44-\mathrm{C} 45 & -7.8(2) & & \end{array}$

Hydrogen-bond geometry $\left(\AA,{ }^{\circ}\right)$

\begin{tabular}{lllll}
\hline$D-\mathrm{H}^{\cdots} A$ & $D-\mathrm{H}$ & $\mathrm{H} \cdots A$ & $D \cdots A$ & $D-\mathrm{H}^{\cdots} A$ \\
\hline $\mathrm{O} 61-\mathrm{H} 61 \cdots \mathrm{N} 2$ & $0.88(3)$ & $2.03(3)$ & $2.900(2)$ & $176(2)$ \\
$\mathrm{N} 423-\mathrm{H} 42 A \cdots \mathrm{O} 61^{\mathrm{i}}$ & $0.84(3)$ & $2.33(3)$ & $3.154(3)$ & $167(3)$ \\
$\mathrm{N} 423-\mathrm{H} 42 B^{\cdots} \mathrm{N} 41$ & $0.85(3)$ & $2.27(3)$ & $2.648(3)$ & $107(2)$ \\
\hline
\end{tabular}

Symmetry code: (i) $-x+1,-y+1,-z+1$.

Ethyl (Z)-2-\{2-[3'-methyl-1'-phenyl-5-(thiophen-2-yl)-5'-(2-methylphenoxy)-3,4-dihydro-1'H,2H-3,4'bipyrazole-2-yl]-4-oxo-4,5-dihydrothiazol-5-ylidene\}acetate (II)

\section{Crystal data}

$\mathrm{C}_{31} \mathrm{H}_{27} \mathrm{~N}_{5} \mathrm{O}_{4} \mathrm{~S}_{2}$

$M_{r}=597.69$

Triclinic, $P \overline{1}$

$a=10.783(2) \AA$

$b=11.683(3) \AA$

$c=13.577(3) \AA$

$\alpha=93.54(2)^{\circ}$

$\beta=105.17(2)^{\circ}$

$\gamma=113.20(2)^{\circ}$

$V=1490.9(6) \AA^{3}$

\section{Data collection}

Oxford Diffraction Xcalibur with Sapphire CCD detector diffractometer

Radiation source: Enhance (Mo) X-ray Source Graphite monochromator

$\omega$ scans

Absorption correction: multi-scan

(CrysAlis RED; Oxford Diffraction, 2009)

$T_{\min }=0.849, T_{\max }=0.987$

$$
Z=2
$$$$
F(000)=624
$$

$D_{\mathrm{x}}=1.331 \mathrm{Mg} \mathrm{m}^{-3}$

Mo $K \alpha$ radiation, $\lambda=0.71073 \AA$

Cell parameters from 6462 reflections

$\theta=2.5-28.2^{\circ}$

$\mu=0.22 \mathrm{~mm}^{-1}$

$T=296 \mathrm{~K}$

Needle, yellow

$0.48 \times 0.12 \times 0.06 \mathrm{~mm}$

10433 measured reflections

5561 independent reflections

1730 reflections with $I>2 \sigma(I)$

$R_{\text {int }}=0.138$

$\theta_{\max }=25.6^{\circ}, \theta_{\text {min }}=2.5^{\circ}$

$h=-7 \rightarrow 13$

$k=-14 \rightarrow 12$

$l=-16 \rightarrow 16$ 


\section{Refinement}

Refinement on $F^{2}$

Least-squares matrix: full

$R\left[F^{2}>2 \sigma\left(F^{2}\right)\right]=0.079$

$w R\left(F^{2}\right)=0.143$

$S=0.88$

5561 reflections

395 parameters

10 restraints
Primary atom site location: difference Fourier map

Hydrogen site location: inferred from neighbouring sites

$\mathrm{H}$-atom parameters constrained

$w=1 /\left[\sigma^{2}\left(F_{\mathrm{o}}^{2}\right)+(0.0319 P)^{2}\right]$ where $P=\left(F_{\mathrm{o}}^{2}+2 F_{\mathrm{c}}{ }^{2}\right) / 3$

$(\Delta / \sigma)_{\max }<0.001$

$\Delta \rho_{\max }=0.26 \mathrm{e} \AA^{-3}$

$\Delta \rho_{\min }=-0.24$ e $\AA^{-3}$

Special details

Experimental. CrysAlis RED, Oxford Diffraction Ltd., 2009 Empirical absorption correction using spherical harmonics, implemented in SCALE3 ABSPACK scaling algorithm.

Geometry. All esds (except the esd in the dihedral angle between two 1.s. planes) are estimated using the full covariance matrix. The cell esds are taken into account individually in the estimation of esds in distances, angles and torsion angles; correlations between esds in cell parameters are only used when they are defined by crystal symmetry. An approximate (isotropic) treatment of cell esds is used for estimating esds involving l.s. planes.

Fractional atomic coordinates and isotropic or equivalent isotropic displacement parameters $\left(\AA^{2}\right)$

\begin{tabular}{llllll}
\hline & $x$ & $y$ & $z$ & $U_{\text {iso }}^{*} / U_{\text {eq }}$ & Occ. $(<1)$ \\
\hline $\mathrm{N} 1$ & $1.0895(5)$ & $0.0030(4)$ & $0.1894(3)$ & $0.0436(12)$ & \\
$\mathrm{N} 2$ & $1.1527(4)$ & $0.1065(4)$ & $0.1476(4)$ & $0.0459(12)$ & \\
$\mathrm{C} 3$ & $1.0468(6)$ & $0.1321(4)$ & $0.0945(4)$ & $0.0430(15)$ & \\
$\mathrm{C} 4$ & $0.9141(5)$ & $0.0459(5)$ & $0.0992(4)$ & $0.0401(14)$ & \\
$\mathrm{C} 5$ & $0.9473(6)$ & $-0.0321(5)$ & $0.1612(4)$ & $0.0424(15)$ & \\
$\mathrm{C} 11$ & $1.1772(6)$ & $-0.0460(5)$ & $0.2534(4)$ & $0.0420(14)$ & \\
$\mathrm{C} 12$ & $1.1198(6)$ & $-0.1658(5)$ & $0.2763(5)$ & $0.0671(17)$ \\
$\mathrm{H} 12$ & 1.0229 & -0.2171 & 0.2490 & $0.0644(18)$ \\
$\mathrm{C} 13$ & $1.2090(7)$ & $-0.2083(5)$ & $0.3410(5)$ & $0.077^{*}$ \\
$\mathrm{H} 13$ & 1.1707 & -0.2886 & 0.3570 & $0.0657(18)$ \\
$\mathrm{C} 14$ & $1.3536(7)$ & $-0.1337(6)$ & $0.3821(5)$ & $0.079^{*}$ \\
$\mathrm{H} 14$ & 1.4123 & -0.1629 & 0.4256 & $0.0596(17)$ \\
$\mathrm{C} 15$ & $1.4082(6)$ & $-0.0162(6)$ & $0.3573(5)$ & $0.071^{*}$ \\
$\mathrm{H} 15$ & 1.5054 & 0.0343 & 0.3832 & $0.0459(14)$ \\
$\mathrm{C} 16$ & $1.3214(6)$ & $0.0286(5)$ & $0.2947(4)$ & $0.055^{*}$ \\
$\mathrm{H} 16$ & 1.3602 & 0.1096 & 0.2801 & $0.0609(17)$ \\
$\mathrm{C} 31$ & $1.0776(5)$ & $0.2433(4)$ & $0.0406(4)$ & $0.091^{*}$ \\
$\mathrm{H} 31 \mathrm{~A}$ & 1.0234 & 0.2153 & -0.0317 & $0.091^{*}$ \\
$\mathrm{H} 31 \mathrm{~B}$ & 1.1770 & 0.2823 & 0.0477 & $0.091^{*}$ \\
$\mathrm{H} 31 \mathrm{C}$ & 1.0520 & 0.3037 & 0.0711 & $0.0455(12)$ \\
$\mathrm{N} 41$ & $0.5871(4)$ & $-0.0182(4)$ & $0.1331(3)$ & $0.0482(13)$ \\
$\mathrm{N} 42$ & $0.7101(4)$ & $0.0797(4)$ & $0.1249(4)$ & $0.0487(16)$ \\
$\mathrm{C} 43$ & $0.7718(6)$ & $0.0410(4)$ & $0.0483(4)$ & $0.058^{*}$ \\
$\mathrm{H} 43$ & 0.7777 & 0.0954 & -0.0040 & $0.0511(16)$ \\
$\mathrm{C} 44$ & $0.6507(5)$ & $-0.0938(4)$ & $-0.0010(4)$ & $0.061^{*}$ \\
$\mathrm{H} 44 \mathrm{~A}$ & 0.6003 & -0.0969 & -0.0724 & $0.061^{*}$ \\
$\mathrm{H} 44 \mathrm{~B}$ & 0.6884 & -0.1568 & 0.0005 &
\end{tabular}




\begin{tabular}{|c|c|c|c|c|c|}
\hline $\mathrm{C} 45$ & $0.5547(5)$ & $-0.1162(5)$ & $0.0651(4)$ & $0.0439(15)$ & \\
\hline S421 & $0.68139(15)$ & $0.21639(13)$ & $0.27511(12)$ & $0.0544(5)$ & \\
\hline $\mathrm{C} 422$ & $0.7658(6)$ & $0.1937(5)$ & $0.1848(5)$ & $0.0505(16)$ & \\
\hline N423 & $0.8776(5)$ & $0.2867(4)$ & $0.1773(4)$ & $0.0558(14)$ & \\
\hline $\mathrm{C} 424$ & $0.9084(6)$ & $0.3951(6)$ & $0.2437(5)$ & $0.0622(18)$ & \\
\hline O424 & $1.0021(4)$ & $0.4986(4)$ & $0.2506(3)$ & $0.0877(15)$ & \\
\hline $\mathrm{C} 425$ & $0.8101(5)$ & $0.3718(5)$ & $0.3113(5)$ & $0.0482(15)$ & \\
\hline $\mathrm{C} 426$ & $0.8307(6)$ & $0.4611(5)$ & $0.3868(5)$ & $0.0636(18)$ & \\
\hline H426 & 0.9061 & 0.5406 & 0.3996 & $0.076^{*}$ & \\
\hline $\mathrm{C} 427$ & $0.7337(7)$ & $0.4347(6)$ & $0.4514(5)$ & 0.0637 (19) & \\
\hline O427 & $0.6309(5)$ & $0.3363(4)$ & $0.4379(3)$ & $0.0842(15)$ & \\
\hline O428 & $0.7767(4)$ & $0.5339(4)$ & 0.5256 & $0.0852(14)$ & \\
\hline C428 & $0.6931(7)$ & $0.5170(6)$ & $0.5969(6)$ & $0.094(2)$ & \\
\hline H48A & 0.7096 & 0.4595 & 0.6416 & $0.112 *$ & \\
\hline H48B & 0.5928 & 0.4809 & 0.5580 & $0.112 *$ & \\
\hline C429 & $0.7352(7)$ & $0.6415(7)$ & $0.6602(6)$ & $0.119(3)$ & \\
\hline H49A & 0.6803 & 0.6314 & 0.7071 & $0.179^{*}$ & \\
\hline H49B & 0.7184 & 0.6980 & 0.6155 & $0.179 *$ & \\
\hline $\mathrm{H} 49 \mathrm{C}$ & 0.8342 & 0.6763 & 0.6992 & $0.179 *$ & \\
\hline S451 & $0.3311(4)$ & -0.2531 & 0.1357 & $0.0655(10)$ & $0.768(6)$ \\
\hline $\mathrm{C} 452$ & $0.4337(6)$ & $-0.2339(5)$ & $0.0544(4)$ & $0.0499(15)$ & $0.768(6)$ \\
\hline C453 & $0.398(3)$ & $-0.339(2)$ & $-0.0103(19)$ & $0.073(3)$ & $0.768(6)$ \\
\hline H453 & 0.4475 & -0.3467 & -0.0552 & $0.087^{*}$ & $0.768(6)$ \\
\hline $\mathrm{C} 454$ & $0.270(2)$ & $-0.4427(17)$ & $-0.002(2)$ & $0.075(5)$ & $0.768(6)$ \\
\hline H454 & 0.2228 & -0.5220 & -0.0449 & $0.090^{*}$ & $0.768(6)$ \\
\hline $\mathrm{C} 455$ & $0.2293(14)$ & $-0.4072(9)$ & $0.0772(15)$ & $0.073(4)$ & $0.768(6)$ \\
\hline H455 & 0.1540 & -0.4614 & 0.0971 & $0.087^{*}$ & $0.768(6)$ \\
\hline S551 & $0.383(3)$ & -0.3635 (19) & $-0.0394(18)$ & $0.073(3)$ & $0.232(6)$ \\
\hline C552 & $0.4337(6)$ & $-0.2339(5)$ & $0.0544(4)$ & $0.0499(15)$ & $0.232(6)$ \\
\hline C553 & $0.349(5)$ & $-0.251(4)$ & $0.112(4)$ & $0.0655(10)$ & $0.232(6)$ \\
\hline H553 & 0.3652 & -0.1924 & 0.1690 & $0.079 *$ & $0.232(6)$ \\
\hline C554 & $0.224(5)$ & -0.375 & $0.073(5)$ & $0.073(4)$ & $0.232(6)$ \\
\hline H554 & 0.1462 & -0.4003 & 0.0973 & $0.087^{*}$ & $0.232(6)$ \\
\hline C555 & $0.239(9)$ & $-0.446(5)$ & $-0.002(8)$ & $0.075(5)$ & $0.232(6)$ \\
\hline H555 & 0.1772 & -0.5306 & -0.0298 & $0.090^{*}$ & $0.232(6)$ \\
\hline O51 & $0.8569(3)$ & -0.1355 & 0.1888 & $0.0513(10)$ & \\
\hline C51 & $0.8251(6)$ & $-0.1159(6)$ & $0.2805(5)$ & $0.0543(16)$ & \\
\hline C52 & $0.7268(7)$ & $-0.2241(7)$ & $0.3002(6)$ & $0.073(2)$ & \\
\hline C53 & $0.6939(8)$ & $-0.2041(10)$ & $0.3902(8)$ & $0.116(3)$ & \\
\hline H53 & 0.6310 & -0.2738 & 0.4086 & $0.140^{*}$ & \\
\hline C54 & $0.7476(11)$ & $-0.0898(13)$ & $0.4529(8)$ & $0.132(4)$ & \\
\hline H54 & 0.7183 & -0.0825 & 0.5107 & $0.158 *$ & \\
\hline C55 & $0.8442(10)$ & $0.0144(10)$ & $0.4315(6)$ & $0.105(3)$ & \\
\hline H55 & 0.8838 & 0.0924 & 0.4756 & $0.125^{*}$ & \\
\hline $\mathrm{C} 56$ & $0.8832(6)$ & $0.0023(6)$ & $0.3421(5)$ & 0.0704 (19) & \\
\hline H56 & 0.9468 & 0.0725 & 0.3247 & $0.084^{*}$ & \\
\hline C57 & $0.6674(7)$ & $-0.3502(6)$ & $0.2333(6)$ & $0.105(3)$ & \\
\hline H57A & 0.5900 & -0.4086 & 0.2528 & $0.158 *$ & \\
\hline
\end{tabular}




$\begin{array}{lllll}\text { H57B } & 0.7399 & -0.3803 & 0.2416 & 0.158^{*} \\ \text { H57C } & 0.6337 & -0.3435 & 0.1620 & 0.158^{*}\end{array}$

Atomic displacement parameters $\left(\AA^{2}\right)$

\begin{tabular}{|c|c|c|c|c|c|c|}
\hline & $U^{11}$ & $U^{22}$ & $U^{33}$ & $U^{12}$ & $U^{13}$ & $U^{23}$ \\
\hline N1 & $0.042(3)$ & $0.044(3)$ & $0.045(3)$ & $0.016(3)$ & $0.016(3)$ & $0.008(2)$ \\
\hline $\mathrm{N} 2$ & $0.043(3)$ & $0.043(3)$ & $0.053(3)$ & $0.011(2)$ & $0.028(3)$ & $0.011(3)$ \\
\hline $\mathrm{C} 3$ & $0.047(4)$ & $0.041(3)$ & $0.046(4)$ & 0.017 (3) & $0.025(3)$ & 0.007 (3) \\
\hline $\mathrm{C} 4$ & $0.039(4)$ & $0.047(3)$ & $0.039(4)$ & $0.020(3)$ & $0.017(3)$ & 0.009 (3) \\
\hline C5 & $0.040(4)$ & $0.042(3)$ & $0.036(4)$ & $0.007(3)$ & $0.016(3)$ & $0.000(3)$ \\
\hline $\mathrm{C} 11$ & 0.040 & 0.045 (3) & $0.037(4)$ & $0.016(3)$ & $0.011(3)$ & $0.001(3)$ \\
\hline $\mathrm{C} 12$ & $0.050(4)$ & 0.048 (4) & $0.065(5)$ & $0.014(3)$ & $0.016(4)$ & 0.009 (3) \\
\hline C13 & $0.076(5)$ & 0.053 (4) & $0.068(5)$ & 0.029 (4) & $0.024(4)$ & 0.019 (4) \\
\hline C14 & $0.062(5)$ & 0.075 (4) & $0.058(5)$ & $0.032(4)$ & $0.010(4)$ & $0.015(4)$ \\
\hline C15 & $0.052(4)$ & 0.068 (4) & $0.050(5)$ & 0.018 (4) & 0.013 (4) & $0.010(4)$ \\
\hline $\mathrm{C} 16$ & $0.051(4)$ & $0.050(3)$ & $0.035(4)$ & $0.015(3)$ & $0.020(3)$ & 0.015 \\
\hline C31 & 0.068 (4) & $0.051(3)$ & $0.067(5)$ & 0.019 (3) & $0.034(4)$ & 0.019 (3) \\
\hline N41 & $0.047(3)$ & 0.042 & $0.047(3)$ & $0.015(2)$ & $0.021(3)$ & $0.012(2)$ \\
\hline N42 & $0.047(3)$ & $0.046(3)$ & $0.057(4)$ & $0.022(2)$ & $0.022(3)$ & $0.006(3)$ \\
\hline $\mathrm{C} 43$ & $0.057(4)$ & $0.051(3)$ & $0.043(4)$ & $0.021(3)$ & $0.027(3)$ & $0.003(3)$ \\
\hline $\mathrm{C} 44$ & $0.043(4)$ & $0.061(4)$ & $0.046(4)$ & $0.021(3)$ & $0.014(3)$ & $0.001(3)$ \\
\hline $\mathrm{C} 45$ & 0.047 (4) & 0.047 (3) & $0.041(4)$ & $0.024(3)$ & $0.013(3)$ & $0.013(3)$ \\
\hline S421 & 0.0588 (11) & $0.0465(8)$ & $0.0573(12)$ & $0.0175(8)$ & $0.0263(9)$ & $0.0045(8)$ \\
\hline $\mathrm{C} 422$ & $0.063(4)$ & $0.051(4)$ & $0.052(4)$ & $0.034(3)$ & $0.027(4)$ & $0.013(3)$ \\
\hline N423 & $0.059(3)$ & $0.042(3)$ & $0.061(4)$ & $0.009(2)$ & $0.030(3)$ & 0.005 \\
\hline $\mathrm{C} 424$ & $0.061(5)$ & $0.055(4)$ & $0.058(5)$ & $0.014(4)$ & $0.018(4)$ & 0.007 (4) \\
\hline $\mathrm{O} 424$ & $0.087(3)$ & $0.056(2)$ & $0.089(4)$ & $-0.008(2)$ & $0.043(3)$ & $-0.005(3)$ \\
\hline $\mathrm{C} 425$ & $0.056(4)$ & $0.038(3)$ & $0.051(4)$ & $0.017(3)$ & $0.021(3)$ & $0.010(3)$ \\
\hline $\mathrm{C} 426$ & $0.074(5)$ & $0.053(4)$ & $0.064(5)$ & $0.020(3)$ & $0.035(4)$ & $0.001(4)$ \\
\hline $\mathrm{C} 427$ & $0.074(5)$ & $0.053(4)$ & $0.052(5)$ & $0.030(4)$ & $0.001(4)$ & $-0.013(4)$ \\
\hline $\mathrm{O} 427$ & $0.093(4)$ & $0.071(3)$ & $0.074(4)$ & $0.018(3)$ & $0.033(3)$ & -0.005 \\
\hline O428 & $0.101(4)$ & $0.074(3)$ & $0.075(4)$ & $0.030(3)$ & $0.036(3)$ & -0.011 \\
\hline $\mathrm{C} 428$ & $0.102(6)$ & $0.107(6)$ & $0.076(6)$ & $0.048(5)$ & $0.033(5)$ & $-0.006(5)$ \\
\hline $\mathrm{C} 429$ & $0.147(7)$ & $0.131(6)$ & $0.083(6)$ & $0.073(6)$ & $0.028(5)$ & $-0.020(5)$ \\
\hline S451 & $0.0732(19)$ & $0.0564(14)$ & $0.068(2)$ & $0.0167(12)$ & $0.0389(13)$ & $0.0163(14)$ \\
\hline C452 & $0.058(4)$ & $0.040(3)$ & $0.048(4)$ & $0.017(3)$ & $0.018(3)$ & $0.004(3)$ \\
\hline C453 & $0.085(6)$ & $0.043(7)$ & $0.053(11)$ & $0.002(5)$ & $0.007(7)$ & $-0.003(5)$ \\
\hline C454 & 0.080 & $0.046(4)$ & $0.066(6)$ & $-0.001(5)$ & $0.017(9)$ & $-0.002(4)$ \\
\hline C455 & $0.083(5)$ & $0.046(7)$ & $0.079(6)$ & $0.016(5)$ & $0.026(5)$ & $0.014(7)$ \\
\hline S551 & $0.085(6)$ & $0.043(7)$ & $0.053(11)$ & $0.002(5)$ & $0.007(7)$ & $-0.003(5)$ \\
\hline C552 & $0.058(4)$ & $0.040(3)$ & $0.048(4)$ & $0.017(3)$ & $0.018(3)$ & $0.004(3)$ \\
\hline C553 & 0.0732 (19) & $0.0564(14)$ & $0.068(2)$ & $0.0167(12)$ & $0.0389(13)$ & $0.0163(14)$ \\
\hline C554 & $0.083(5)$ & $0.046(7)$ & $0.079(6)$ & $0.016(5)$ & $0.026(5)$ & $0.014(7)$ \\
\hline C555 & $0.080(13)$ & $0.046(4)$ & $0.066(6)$ & $-0.001(5)$ & $0.017(9)$ & $-0.002(4)$ \\
\hline O51 & $0.049(2)$ & $0.056(2)$ & $0.048(3)$ & 0.0145 (19) & $0.024(2)$ & $0.010(2)$ \\
\hline C51 & $0.058(4)$ & $0.075(4)$ & $0.042(5)$ & $0.037(4)$ & $0.020(4)$ & $0.020(4)$ \\
\hline $\mathrm{C} 52$ & $0.065(5)$ & $0.110(6)$ & $0.068(6)$ & $0.041(5)$ & $0.042(4)$ & $0.065(5)$ \\
\hline
\end{tabular}




\begin{tabular}{lllllll} 
C53 & $0.098(7)$ & $0.169(9)$ & $0.110(10)$ & $0.054(7)$ & $0.068(7)$ & $0.081(7)$ \\
C54 & $0.140(10)$ & $0.248(15)$ & $0.078(8)$ & $0.125(10)$ & $0.068(7)$ & $0.074(9)$ \\
C55 & $0.136(8)$ & $0.182(9)$ & $0.049(6)$ & $0.107(7)$ & $0.047(5)$ & $0.035(6)$ \\
C56 & $0.077(5)$ & $0.102(5)$ & $0.055(5)$ & $0.052(4)$ & $0.034(4)$ & $0.019(4)$ \\
C57 & $0.092(6)$ & $0.082(5)$ & $0.129(7)$ & $0.013(4)$ & $0.041(5)$ & $0.070(5)$ \\
\hline
\end{tabular}

Geometric parameters $\left(A,{ }^{\circ}\right)$

\begin{tabular}{|c|c|c|c|}
\hline $\mathrm{N} 1-\mathrm{C} 5$ & $1.358(6)$ & $\mathrm{C} 426-\mathrm{C} 427$ & $1.489(8)$ \\
\hline $\mathrm{N} 1-\mathrm{N} 2$ & $1.374(5)$ & $\mathrm{C} 426-\mathrm{H} 426$ & 0.9300 \\
\hline $\mathrm{N} 1-\mathrm{C} 11$ & $1.426(6)$ & $\mathrm{C} 427-\mathrm{O} 427$ & $1.203(6)$ \\
\hline $\mathrm{N} 2-\mathrm{C} 3$ & $1.333(6)$ & $\mathrm{C} 427-\mathrm{O} 428$ & $1.327(6)$ \\
\hline $\mathrm{C} 3-\mathrm{C} 4$ & $1.409(6)$ & $\mathrm{O} 428-\mathrm{C} 428$ & $1.458(7)$ \\
\hline $\mathrm{C} 3-\mathrm{C} 31$ & $1.497(6)$ & $\mathrm{C} 428-\mathrm{C} 429$ & $1.477(7)$ \\
\hline $\mathrm{C} 4-\mathrm{C} 5$ & $1.364(7)$ & $\mathrm{C} 428-\mathrm{H} 48 \mathrm{~A}$ & 0.9700 \\
\hline $\mathrm{C} 4-\mathrm{C} 43$ & $1.485(6)$ & $\mathrm{C} 428-\mathrm{H} 48 \mathrm{~B}$ & 0.9700 \\
\hline $\mathrm{C} 5-\mathrm{O} 51$ & $1.367(6)$ & $\mathrm{C} 429-\mathrm{H} 49 \mathrm{~A}$ & 0.9600 \\
\hline $\mathrm{C} 11-\mathrm{C} 16$ & $1.382(6)$ & $\mathrm{C} 429-\mathrm{H} 49 \mathrm{~B}$ & 0.9600 \\
\hline $\mathrm{C} 11-\mathrm{C} 12$ & $1.382(7)$ & $\mathrm{C} 429-\mathrm{H} 49 \mathrm{C}$ & 0.9600 \\
\hline $\mathrm{C} 12-\mathrm{C} 13$ & $1.390(7)$ & $\mathrm{S} 451-\mathrm{C} 455$ & $1.699(9)$ \\
\hline $\mathrm{C} 12-\mathrm{H} 12$ & 0.9300 & $\mathrm{~S} 451-\mathrm{C} 452$ & $1.723(6)$ \\
\hline $\mathrm{C} 13-\mathrm{C} 14$ & $1.384(7)$ & $\mathrm{C} 452-\mathrm{C} 453$ & $1.320(16)$ \\
\hline $\mathrm{C} 13-\mathrm{H} 13$ & 0.9300 & $\mathrm{C} 453-\mathrm{C} 454$ & $1.47(2)$ \\
\hline $\mathrm{C} 14-\mathrm{C} 15$ & $1.367(7)$ & $\mathrm{C} 453-\mathrm{H} 453$ & 0.9300 \\
\hline $\mathrm{C} 14-\mathrm{H} 14$ & 0.9300 & $\mathrm{C} 454-\mathrm{C} 455$ & $1.359(10)$ \\
\hline $\mathrm{C} 15-\mathrm{C} 16$ & $1.377(7)$ & $\mathrm{C} 454-\mathrm{H} 454$ & 0.9300 \\
\hline C15-H15 & 0.9300 & $\mathrm{C} 455-\mathrm{H} 455$ & 0.9300 \\
\hline $\mathrm{C} 16-\mathrm{H} 16$ & 0.9300 & S551-C555 & $1.698(14)$ \\
\hline $\mathrm{C} 31-\mathrm{H} 31 \mathrm{~A}$ & 0.9600 & C553-C554 & $1.48(2)$ \\
\hline $\mathrm{C} 31-\mathrm{H} 31 \mathrm{~B}$ & 0.9600 & C553-H553 & 0.9300 \\
\hline $\mathrm{C} 31-\mathrm{H} 31 \mathrm{C}$ & 0.9600 & C554-C555 & $1.359(13)$ \\
\hline $\mathrm{N} 41-\mathrm{C} 45$ & $1.293(5)$ & C554-H554 & 0.9300 \\
\hline $\mathrm{N} 41-\mathrm{N} 42$ & $1.405(5)$ & С555-H555 & 0.9300 \\
\hline $\mathrm{N} 42-\mathrm{C} 422$ & $1.333(5)$ & $\mathrm{O} 51-\mathrm{C} 51$ & $1.405(6)$ \\
\hline $\mathrm{N} 42-\mathrm{C} 43$ & $1.507(6)$ & $\mathrm{C} 51-\mathrm{C} 56$ & $1.379(7)$ \\
\hline $\mathrm{C} 43-\mathrm{C} 44$ & $1.557(6)$ & $\mathrm{C} 51-\mathrm{C} 52$ & $1.387(8)$ \\
\hline $\mathrm{C} 43-\mathrm{H} 43$ & 0.9800 & $\mathrm{C} 52-\mathrm{C} 53$ & $1.390(10)$ \\
\hline $\mathrm{C} 44-\mathrm{C} 45$ & $1.499(7)$ & $\mathrm{C} 52-\mathrm{C} 57$ & $1.481(8)$ \\
\hline $\mathrm{C} 44-\mathrm{H} 44 \mathrm{~A}$ & 0.9700 & $\mathrm{C} 53-\mathrm{C} 54$ & $1.355(10)$ \\
\hline $\mathrm{C} 44-\mathrm{H} 44 \mathrm{~B}$ & 0.9700 & C53-H53 & 0.9300 \\
\hline $\mathrm{C} 45-\mathrm{C} 452$ & $1.441(6)$ & $\mathrm{C} 54-\mathrm{C} 55$ & $1.362(11)$ \\
\hline $\mathrm{S} 421-\mathrm{C} 425$ & $1.736(5)$ & C54-H54 & 0.9300 \\
\hline $\mathrm{S} 421-\mathrm{C} 422$ & $1.771(5)$ & $\mathrm{C} 55-\mathrm{C} 56$ & $1.400(9)$ \\
\hline $\mathrm{C} 422-\mathrm{N} 423$ & $1.300(6)$ & C55-H55 & 0.9300 \\
\hline $\mathrm{N} 423-\mathrm{C} 424$ & $1.378(6)$ & C56-H56 & 0.9300 \\
\hline $\mathrm{C} 424-\mathrm{O} 424$ & $1.211(6)$ & C57-H57A & 0.9600 \\
\hline $\mathrm{C} 424-\mathrm{C} 425$ & $1.534(7)$ & C57-H57B & 0.9600 \\
\hline $\mathrm{C} 425-\mathrm{C} 426$ & $1.325(6)$ & C57-H57C & 0.9600 \\
\hline
\end{tabular}




\begin{tabular}{|c|c|c|c|}
\hline $\mathrm{C} 5-\mathrm{N} 1-\mathrm{N} 2$ & $109.6(4)$ & $\mathrm{C} 424-\mathrm{C} 425-\mathrm{S} 421$ & $109.7(4)$ \\
\hline $\mathrm{C} 5-\mathrm{N} 1-\mathrm{C} 11$ & $131.7(5)$ & $\mathrm{C} 425-\mathrm{C} 426-\mathrm{C} 427$ & $120.3(5)$ \\
\hline $\mathrm{N} 2-\mathrm{N} 1-\mathrm{C} 11$ & $118.6(4)$ & $\mathrm{C} 425-\mathrm{C} 426-\mathrm{H} 426$ & 119.9 \\
\hline $\mathrm{C} 3-\mathrm{N} 2-\mathrm{N} 1$ & $105.2(4)$ & $\mathrm{C} 427-\mathrm{C} 426-\mathrm{H} 426$ & 119.9 \\
\hline $\mathrm{N} 2-\mathrm{C} 3-\mathrm{C} 4$ & $112.1(5)$ & $\mathrm{O} 427-\mathrm{C} 427-\mathrm{O} 428$ & $125.0(7)$ \\
\hline $\mathrm{N} 2-\mathrm{C} 3-\mathrm{C} 31$ & $120.0(5)$ & $\mathrm{O} 427-\mathrm{C} 427-\mathrm{C} 426$ & $124.2(6)$ \\
\hline $\mathrm{C} 4-\mathrm{C} 3-\mathrm{C} 31$ & $127.9(6)$ & $\mathrm{O} 428-\mathrm{C} 427-\mathrm{C} 426$ & $110.7(6)$ \\
\hline $\mathrm{C} 5-\mathrm{C} 4-\mathrm{C} 3$ & $103.6(5)$ & $\mathrm{C} 427-\mathrm{O} 428-\mathrm{C} 428$ & $115.7(5)$ \\
\hline $\mathrm{C} 5-\mathrm{C} 4-\mathrm{C} 43$ & $128.2(5)$ & $\mathrm{O} 428-\mathrm{C} 428-\mathrm{C} 429$ & $108.9(6)$ \\
\hline $\mathrm{C} 3-\mathrm{C} 4-\mathrm{C} 43$ & $128.2(5)$ & $\mathrm{O} 428-\mathrm{C} 428-\mathrm{H} 48 \mathrm{~A}$ & 109.9 \\
\hline $\mathrm{N} 1-\mathrm{C} 5-\mathrm{C} 4$ & $109.4(5)$ & $\mathrm{C} 429-\mathrm{C} 428-\mathrm{H} 48 \mathrm{~A}$ & 109.9 \\
\hline $\mathrm{N} 1-\mathrm{C} 5-\mathrm{O} 51$ & $122.3(5)$ & $\mathrm{O} 428-\mathrm{C} 428-\mathrm{H} 48 \mathrm{~B}$ & 109.9 \\
\hline $\mathrm{C} 4-\mathrm{C} 5-\mathrm{O} 51$ & $128.2(5)$ & $\mathrm{C} 429-\mathrm{C} 428-\mathrm{H} 48 \mathrm{~B}$ & 109.9 \\
\hline $\mathrm{C} 16-\mathrm{C} 11-\mathrm{C} 12$ & $119.6(5)$ & $\mathrm{H} 48 \mathrm{~A}-\mathrm{C} 428-\mathrm{H} 48 \mathrm{~B}$ & 108.3 \\
\hline $\mathrm{C} 16-\mathrm{C} 11-\mathrm{N} 1$ & $119.3(5)$ & $\mathrm{C} 428-\mathrm{C} 429-\mathrm{H} 49 \mathrm{~A}$ & 109.5 \\
\hline $\mathrm{C} 12-\mathrm{C} 11-\mathrm{N} 1$ & $121.1(5)$ & $\mathrm{C} 428-\mathrm{C} 429-\mathrm{H} 49 \mathrm{~B}$ & 109.5 \\
\hline $\mathrm{C} 11-\mathrm{C} 12-\mathrm{C} 13$ & $119.0(5)$ & $\mathrm{H} 49 \mathrm{~A}-\mathrm{C} 429-\mathrm{H} 49 \mathrm{~B}$ & 109.5 \\
\hline $\mathrm{C} 11-\mathrm{C} 12-\mathrm{H} 12$ & 120.5 & $\mathrm{C} 428-\mathrm{C} 429-\mathrm{H} 49 \mathrm{C}$ & 109.5 \\
\hline $\mathrm{C} 13-\mathrm{C} 12-\mathrm{H} 12$ & 120.5 & $\mathrm{H} 49 \mathrm{~A}-\mathrm{C} 429-\mathrm{H} 49 \mathrm{C}$ & 109.5 \\
\hline $\mathrm{C} 14-\mathrm{C} 13-\mathrm{C} 12$ & $121.4(6)$ & $\mathrm{H} 49 \mathrm{~B}-\mathrm{C} 429-\mathrm{H} 49 \mathrm{C}$ & 109.5 \\
\hline $\mathrm{C} 14-\mathrm{C} 13-\mathrm{H} 13$ & 119.3 & $\mathrm{C} 455-\mathrm{S} 451-\mathrm{C} 452$ & $91.1(5)$ \\
\hline $\mathrm{C} 12-\mathrm{C} 13-\mathrm{H} 13$ & 119.3 & $\mathrm{C} 453-\mathrm{C} 452-\mathrm{C} 45$ & $124.6(12)$ \\
\hline $\mathrm{C} 15-\mathrm{C} 14-\mathrm{C} 13$ & $118.5(6)$ & $\mathrm{C} 453-\mathrm{C} 452-\mathrm{S} 451$ & $113.6(11)$ \\
\hline $\mathrm{C} 15-\mathrm{C} 14-\mathrm{H} 14$ & 120.7 & $\mathrm{C} 45-\mathrm{C} 452-\mathrm{S} 451$ & $121.6(4)$ \\
\hline $\mathrm{C} 13-\mathrm{C} 14-\mathrm{H} 14$ & 120.7 & $\mathrm{C} 452-\mathrm{C} 453-\mathrm{C} 454$ & $111.2(14)$ \\
\hline $\mathrm{C} 14-\mathrm{C} 15-\mathrm{C} 16$ & $121.0(6)$ & $\mathrm{C} 452-\mathrm{C} 453-\mathrm{H} 453$ & 124.4 \\
\hline $\mathrm{C} 14-\mathrm{C} 15-\mathrm{H} 15$ & 119.5 & $\mathrm{C} 454-\mathrm{C} 453-\mathrm{H} 453$ & 124.4 \\
\hline $\mathrm{C} 16-\mathrm{C} 15-\mathrm{H} 15$ & 119.5 & $\mathrm{C} 455-\mathrm{C} 454-\mathrm{C} 453$ & $111.1(10)$ \\
\hline $\mathrm{C} 15-\mathrm{C} 16-\mathrm{C} 11$ & $120.4(5)$ & $\mathrm{C} 455-\mathrm{C} 454-\mathrm{H} 454$ & 124.5 \\
\hline $\mathrm{C} 15-\mathrm{C} 16-\mathrm{H} 16$ & 119.8 & $\mathrm{C} 453-\mathrm{C} 454-\mathrm{H} 454$ & 124.5 \\
\hline $\mathrm{C} 11-\mathrm{C} 16-\mathrm{H} 16$ & 119.8 & $\mathrm{C} 454-\mathrm{C} 455-\mathrm{S} 451$ & $112.8(10)$ \\
\hline $\mathrm{C} 3-\mathrm{C} 31-\mathrm{H} 31 \mathrm{~A}$ & 109.5 & $\mathrm{C} 454-\mathrm{C} 455-\mathrm{H} 455$ & 123.6 \\
\hline $\mathrm{C} 3-\mathrm{C} 31-\mathrm{H} 31 \mathrm{~B}$ & 109.5 & $\mathrm{~S} 451-\mathrm{C} 455-\mathrm{H} 455$ & 123.6 \\
\hline $\mathrm{H} 31 \mathrm{~A}-\mathrm{C} 31-\mathrm{H} 31 \mathrm{~B}$ & 109.5 & C554-C553-H553 & 124.6 \\
\hline $\mathrm{C} 3-\mathrm{C} 31-\mathrm{H} 31 \mathrm{C}$ & 109.5 & C555-C554-C553 & $111.3(16)$ \\
\hline $\mathrm{H} 31 \mathrm{~A}-\mathrm{C} 31-\mathrm{H} 31 \mathrm{C}$ & 109.5 & C555-C554-H554 & 124.4 \\
\hline $\mathrm{H} 31 \mathrm{~B}-\mathrm{C} 31-\mathrm{H} 31 \mathrm{C}$ & 109.5 & C553-C554-H554 & 124.4 \\
\hline $\mathrm{C} 45-\mathrm{N} 41-\mathrm{N} 42$ & 107.5 & $\mathrm{C} 554-\mathrm{C} 555-\mathrm{S} 551$ & $112.2(17)$ \\
\hline $\mathrm{C} 422-\mathrm{N} 42-\mathrm{N} 41$ & $120.5(4)$ & C554-C555-H555 & 123.9 \\
\hline $\mathrm{C} 422-\mathrm{N} 42-\mathrm{C} 43$ & $125.3(4)$ & S551-C555-H555 & 123.9 \\
\hline $\mathrm{N} 41-\mathrm{N} 42-\mathrm{C} 43$ & $114.2(4)$ & $\mathrm{C} 5-\mathrm{O} 51-\mathrm{C} 51$ & $117.5(4)$ \\
\hline $\mathrm{C} 4-\mathrm{C} 43-\mathrm{N} 42$ & 112.5 & $\mathrm{C} 56-\mathrm{C} 51-\mathrm{C} 52$ & $123.9(6)$ \\
\hline $\mathrm{C} 4-\mathrm{C} 43-\mathrm{C} 44$ & $115.7(4)$ & $\mathrm{C} 56-\mathrm{C} 51-\mathrm{O} 51$ & $122.0(6)$ \\
\hline $\mathrm{N} 42-\mathrm{C} 43-\mathrm{C} 44$ & $98.8(4)$ & $\mathrm{C} 52-\mathrm{C} 51-\mathrm{O} 51$ & $114.0(6)$ \\
\hline $\mathrm{C} 4-\mathrm{C} 43-\mathrm{H} 43$ & 109.8 & $\mathrm{C} 51-\mathrm{C} 52-\mathrm{C} 53$ & $114.0(7)$ \\
\hline $\mathrm{N} 42-\mathrm{C} 43-\mathrm{H} 43$ & 109.8 & $\mathrm{C} 51-\mathrm{C} 52-\mathrm{C} 57$ & $122.7(7)$ \\
\hline $\mathrm{C} 44-\mathrm{C} 43-\mathrm{H} 43$ & 109.8 & $\mathrm{C} 53-\mathrm{C} 52-\mathrm{C} 57$ & $123.3(7)$ \\
\hline
\end{tabular}




\begin{tabular}{|c|c|c|c|}
\hline $\mathrm{C} 45-\mathrm{C} 44-\mathrm{C} 43$ & $104.6(4)$ & $\mathrm{C} 54-\mathrm{C} 53-\mathrm{C} 52$ & $124.3(10)$ \\
\hline $\mathrm{C} 45-\mathrm{C} 44-\mathrm{H} 44 \mathrm{~A}$ & 110.8 & $\mathrm{C} 54-\mathrm{C} 53-\mathrm{H} 53$ & 117.8 \\
\hline $\mathrm{C} 43-\mathrm{C} 44-\mathrm{H} 44 \mathrm{~A}$ & 110.8 & $\mathrm{C} 52-\mathrm{C} 53-\mathrm{H} 53$ & 117.8 \\
\hline $\mathrm{C} 45-\mathrm{C} 44-\mathrm{H} 44 \mathrm{~B}$ & 110.8 & $\mathrm{C} 53-\mathrm{C} 54-\mathrm{C} 55$ & $120.1(11)$ \\
\hline $\mathrm{C} 43-\mathrm{C} 44-\mathrm{H} 44 \mathrm{~B}$ & 110.8 & $\mathrm{C} 53-\mathrm{C} 54-\mathrm{H} 54$ & 119.9 \\
\hline $\mathrm{H} 44 \mathrm{~A}-\mathrm{C} 44-\mathrm{H} 44 \mathrm{~B}$ & 108.9 & C55-C54-H54 & 119.9 \\
\hline $\mathrm{N} 41-\mathrm{C} 45-\mathrm{C} 452$ & $121.4(5)$ & $\mathrm{C} 54-\mathrm{C} 55-\mathrm{C} 56$ & $119.1(9)$ \\
\hline $\mathrm{N} 41-\mathrm{C} 45-\mathrm{C} 44$ & $114.0(5)$ & C54-C55-H55 & 120.5 \\
\hline $\mathrm{C} 452-\mathrm{C} 45-\mathrm{C} 44$ & $124.5(5)$ & $\mathrm{C} 56-\mathrm{C} 55-\mathrm{H} 55$ & 120.5 \\
\hline $\mathrm{C} 425-\mathrm{S} 421-\mathrm{C} 422$ & $87.3(3)$ & $\mathrm{C} 51-\mathrm{C} 56-\mathrm{C} 55$ & $118.5(7)$ \\
\hline $\mathrm{N} 423-\mathrm{C} 422-\mathrm{N} 42$ & $121.8(5)$ & $\mathrm{C} 51-\mathrm{C} 56-\mathrm{H} 56$ & 120.7 \\
\hline $\mathrm{N} 423-\mathrm{C} 422-\mathrm{S} 421$ & $120.3(4)$ & $\mathrm{C} 55-\mathrm{C} 56-\mathrm{H} 56$ & 120.7 \\
\hline $\mathrm{N} 42-\mathrm{C} 422-\mathrm{S} 421$ & $117.9(4)$ & $\mathrm{C} 52-\mathrm{C} 57-\mathrm{H} 57 \mathrm{~A}$ & 109.5 \\
\hline $\mathrm{C} 422-\mathrm{N} 423-\mathrm{C} 424$ & $110.0(5)$ & C52-C57-H57B & 109.5 \\
\hline $\mathrm{O} 424-\mathrm{C} 424-\mathrm{N} 423$ & $125.4(6)$ & $\mathrm{H} 57 \mathrm{~A}-\mathrm{C} 57-\mathrm{H} 57 \mathrm{~B}$ & 109.5 \\
\hline $\mathrm{O} 424-\mathrm{C} 424-\mathrm{C} 425$ & $122.0(6)$ & $\mathrm{C} 52-\mathrm{C} 57-\mathrm{H} 57 \mathrm{C}$ & 109.5 \\
\hline N423-C424-C425 & $112.5(5)$ & $\mathrm{H} 57 \mathrm{~A}-\mathrm{C} 57-\mathrm{H} 57 \mathrm{C}$ & 109.5 \\
\hline $\mathrm{C} 426-\mathrm{C} 425-\mathrm{C} 424$ & $121.8(5)$ & $\mathrm{H} 57 \mathrm{~B}-\mathrm{C} 57-\mathrm{H} 57 \mathrm{C}$ & 109.5 \\
\hline $\mathrm{C} 426-\mathrm{C} 425-\mathrm{S} 421$ & $128.4(5)$ & & \\
\hline $\mathrm{C} 5-\mathrm{N} 1-\mathrm{N} 2-\mathrm{C} 3$ & $-0.1(5)$ & $\mathrm{C} 43-\mathrm{N} 42-\mathrm{C} 422-\mathrm{S} 421$ & $-176.4(4)$ \\
\hline $\mathrm{C} 11-\mathrm{N} 1-\mathrm{N} 2-\mathrm{C} 3$ & $-178.6(4)$ & $\mathrm{C} 425-\mathrm{S} 421-\mathrm{C} 422-\mathrm{N} 423$ & $-0.2(5)$ \\
\hline $\mathrm{N} 1-\mathrm{N} 2-\mathrm{C} 3-\mathrm{C} 4$ & $-1.0(5)$ & $\mathrm{C} 425-\mathrm{S} 421-\mathrm{C} 422-\mathrm{N} 42$ & $-179.4(4)$ \\
\hline $\mathrm{N} 1-\mathrm{N} 2-\mathrm{C} 3-\mathrm{C} 31$ & $178.2(4)$ & $\mathrm{N} 42-\mathrm{C} 422-\mathrm{N} 423-\mathrm{C} 424$ & $176.8(5)$ \\
\hline $\mathrm{N} 2-\mathrm{C} 3-\mathrm{C} 4-\mathrm{C} 5$ & $1.6(5)$ & $\mathrm{S} 421-\mathrm{C} 422-\mathrm{N} 423-\mathrm{C} 424$ & $-2.4(7)$ \\
\hline $\mathrm{C} 31-\mathrm{C} 3-\mathrm{C} 4-\mathrm{C} 5$ & $-177.5(5)$ & $\mathrm{C} 422-\mathrm{N} 423-\mathrm{C} 424-\mathrm{O} 424$ & $-176.2(6)$ \\
\hline $\mathrm{N} 2-\mathrm{C} 3-\mathrm{C} 4-\mathrm{C} 43$ & $-178.3(4)$ & $\mathrm{C} 422-\mathrm{N} 423-\mathrm{C} 424-\mathrm{C} 425$ & $4.1(7)$ \\
\hline $\mathrm{C} 31-\mathrm{C} 3-\mathrm{C} 4-\mathrm{C} 43$ & $2.7(8)$ & $\mathrm{O} 424-\mathrm{C} 424-\mathrm{C} 425-\mathrm{C} 426$ & $-6.1(9)$ \\
\hline $\mathrm{N} 2-\mathrm{N} 1-\mathrm{C} 5-\mathrm{C} 4$ & $1.1(5)$ & $\mathrm{N} 423-\mathrm{C} 424-\mathrm{C} 425-\mathrm{C} 426$ & $173.5(5)$ \\
\hline $\mathrm{C} 11-\mathrm{N} 1-\mathrm{C} 5-\mathrm{C} 4$ & $179.4(4)$ & $\mathrm{O} 424-\mathrm{C} 424-\mathrm{C} 425-\mathrm{S} 421$ & $176.0(5)$ \\
\hline $\mathrm{N} 2-\mathrm{N} 1-\mathrm{C} 5-\mathrm{O} 51$ & $177.6(4)$ & $\mathrm{N} 423-\mathrm{C} 424-\mathrm{C} 425-\mathrm{S} 421$ & $-4.3(6)$ \\
\hline $\mathrm{C} 11-\mathrm{N} 1-\mathrm{C} 5-\mathrm{O} 51$ & $-4.2(7)$ & $\mathrm{C} 422-\mathrm{S} 421-\mathrm{C} 425-\mathrm{C} 426$ & $-175.3(6)$ \\
\hline $\mathrm{C} 3-\mathrm{C} 4-\mathrm{C} 5-\mathrm{N} 1$ & $-1.6(5)$ & $\mathrm{C} 422-\mathrm{S} 421-\mathrm{C} 425-\mathrm{C} 424$ & $2.4(4)$ \\
\hline $\mathrm{C} 43-\mathrm{C} 4-\mathrm{C} 5-\mathrm{N} 1$ & $178.3(4)$ & $\mathrm{C} 424-\mathrm{C} 425-\mathrm{C} 426-\mathrm{C} 427$ & $-179.5(6)$ \\
\hline $\mathrm{C} 3-\mathrm{C} 4-\mathrm{C} 5-\mathrm{O} 51$ & $-177.8(5)$ & $\mathrm{S} 421-\mathrm{C} 425-\mathrm{C} 426-\mathrm{C} 427$ & $-2.0(8)$ \\
\hline $\mathrm{C} 43-\mathrm{C} 4-\mathrm{C} 5-\mathrm{O} 51$ & $2.1(8)$ & $\mathrm{C} 425-\mathrm{C} 426-\mathrm{C} 427-\mathrm{O} 427$ & $-2.8(10)$ \\
\hline $\mathrm{C} 5-\mathrm{N} 1-\mathrm{C} 11-\mathrm{C} 16$ & $-160.7(5)$ & $\mathrm{C} 425-\mathrm{C} 426-\mathrm{C} 427-\mathrm{O} 428$ & $176.9(5)$ \\
\hline $\mathrm{N} 2-\mathrm{N} 1-\mathrm{C} 11-\mathrm{C} 16$ & $17.5(6)$ & $\mathrm{O} 427-\mathrm{C} 427-\mathrm{O} 428-\mathrm{C} 428$ & $2.3(9)$ \\
\hline $\mathrm{C} 5-\mathrm{N} 1-\mathrm{C} 11-\mathrm{C} 12$ & $18.0(7)$ & $\mathrm{C} 426-\mathrm{C} 427-\mathrm{O} 428-\mathrm{C} 428$ & $-177.4(5)$ \\
\hline $\mathrm{N} 2-\mathrm{N} 1-\mathrm{C} 11-\mathrm{C} 12$ & $-163.8(4)$ & $\mathrm{C} 427-\mathrm{O} 428-\mathrm{C} 428-\mathrm{C} 429$ & $-168.6(5)$ \\
\hline $\mathrm{C} 16-\mathrm{C} 11-\mathrm{C} 12-\mathrm{C} 13$ & $0.2(8)$ & $\mathrm{N} 41-\mathrm{C} 45-\mathrm{C} 452-\mathrm{C} 453$ & $-177.0(19)$ \\
\hline $\mathrm{N} 1-\mathrm{C} 11-\mathrm{C} 12-\mathrm{C} 13$ & $-178.5(4)$ & $\mathrm{C} 44-\mathrm{C} 45-\mathrm{C} 452-\mathrm{C} 453$ & $5(2)$ \\
\hline $\mathrm{C} 11-\mathrm{C} 12-\mathrm{C} 13-\mathrm{C} 14$ & $-0.4(8)$ & $\mathrm{N} 41-\mathrm{C} 45-\mathrm{C} 452-\mathrm{S} 451$ & $-3.3(7)$ \\
\hline $\mathrm{C} 12-\mathrm{C} 13-\mathrm{C} 14-\mathrm{C} 15$ & $-0.3(9)$ & $\mathrm{C} 44-\mathrm{C} 45-\mathrm{C} 452-\mathrm{S} 451$ & $178.4(5)$ \\
\hline $\mathrm{C} 13-\mathrm{C} 14-\mathrm{C} 15-\mathrm{C} 16$ & $1.3(9)$ & $\mathrm{C} 455-\mathrm{S} 451-\mathrm{C} 452-\mathrm{C} 453$ & $-2.9(18)$ \\
\hline $\mathrm{C} 14-\mathrm{C} 15-\mathrm{C} 16-\mathrm{C} 11$ & $-1.6(8)$ & $\mathrm{C} 455-\mathrm{S} 451-\mathrm{C} 452-\mathrm{C} 45$ & $-177.2(8)$ \\
\hline $\mathrm{C} 12-\mathrm{C} 11-\mathrm{C} 16-\mathrm{C} 15$ & $0.8(7)$ & $\mathrm{C} 45-\mathrm{C} 452-\mathrm{C} 453-\mathrm{C} 454$ & $180(2)$ \\
\hline $\mathrm{N} 1-\mathrm{C} 11-\mathrm{C} 16-\mathrm{C} 15$ & $179.5(5)$ & $\mathrm{S} 451-\mathrm{C} 452-\mathrm{C} 453-\mathrm{C} 454$ & $5(3)$ \\
\hline
\end{tabular}




$\begin{array}{ll}\mathrm{C} 45-\mathrm{N} 41-\mathrm{N} 42-\mathrm{C} 422 & 178.4(5) \\ \mathrm{C} 45-\mathrm{N} 41-\mathrm{N} 42-\mathrm{C} 43 & -4.1(6) \\ \mathrm{C} 5-\mathrm{C} 4-\mathrm{C} 43-\mathrm{N} 42 & 70.7(6) \\ \mathrm{C} 3-\mathrm{C} 4-\mathrm{C} 43-\mathrm{N} 42 & -109.5(6) \\ \mathrm{C} 5-\mathrm{C} 4-\mathrm{C} 43-\mathrm{C} 44 & -41.9(7) \\ \mathrm{C} 3-\mathrm{C} 4-\mathrm{C} 43-\mathrm{C} 44 & 138.0(5) \\ \mathrm{C} 422-\mathrm{N} 42-\mathrm{C} 43-\mathrm{C} 4 & 62.7(6) \\ \mathrm{N} 41-\mathrm{N} 42-\mathrm{C} 43-\mathrm{C} 4 & -114.6(5) \\ \mathrm{C} 422-\mathrm{N} 42-\mathrm{C} 43-\mathrm{C} 44 & -174.7(5) \\ \mathrm{N} 41-\mathrm{N} 42-\mathrm{C} 43-\mathrm{C} 44 & 8.0(5) \\ \mathrm{C} 4-\mathrm{C} 43-\mathrm{C} 44-\mathrm{C} 45 & 111.9(5) \\ \mathrm{N} 42-\mathrm{C} 43-\mathrm{C} 44-\mathrm{C} 45 & -8.3(5) \\ \mathrm{N} 42-\mathrm{N} 41-\mathrm{C} 45-\mathrm{C} 452 & 179.2(5) \\ \mathrm{N} 42-\mathrm{N} 41-\mathrm{C} 45-\mathrm{C} 44 & -2.3(6) \\ \mathrm{C} 43-\mathrm{C} 44-\mathrm{C} 45-\mathrm{N} 41 & 7.3(6) \\ \mathrm{C} 43-\mathrm{C} 44-\mathrm{C} 45-\mathrm{C} 452 & -174.3(5) \\ \mathrm{N} 41-\mathrm{N} 42-\mathrm{C} 422-\mathrm{N} 423 & -178.4(5) \\ \mathrm{C} 43-\mathrm{N} 42-\mathrm{C} 422-\mathrm{N} 423 & 4.4(8) \\ \mathrm{N} 41-\mathrm{N} 42-\mathrm{C} 422-\mathrm{S} 421 & 0.7(6)\end{array}$

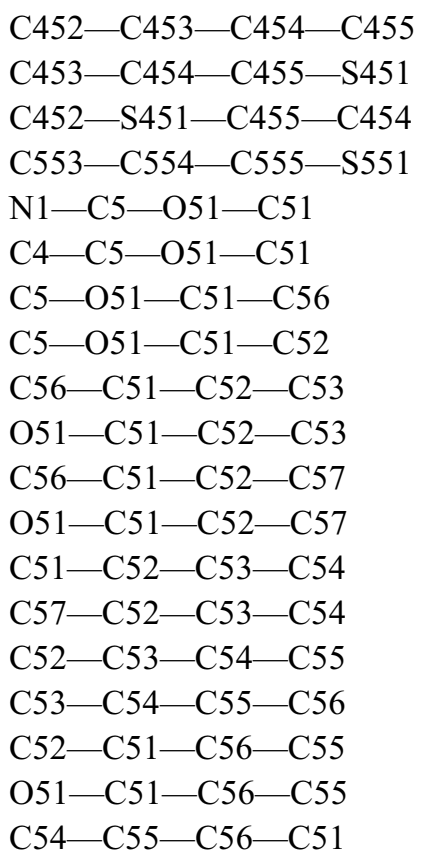

$-6(4)$

4 (4)

$-1(2)$

$-8(12)$

$92.8(5)$

$-91.4(6)$

$-0.8(7)$

$176.7(5)$

$-1.7(9)$

$-179.1(5)$

$-179.3(6)$

$3.3(8)$

$2.1(12)$

$179.6(8)$

$-2.4(15)$

$2.2(14)$

$1.7(9)$

$178.9(5)$

$-1.9(11)$

Hydrogen-bond geometry $\left(\AA,{ }^{\circ}\right)$

\begin{tabular}{lllll}
\hline$D-\mathrm{H} \cdots A$ & $D-\mathrm{H}$ & $\mathrm{H} \cdots A$ & $D \cdots A$ & $D-\mathrm{H} \cdots A$ \\
\hline $\mathrm{C} 13-\mathrm{H} 13 \cdots \mathrm{O} 424^{\mathrm{i}}$ & 0.93 & 2.49 & $3.200(7)$ & 133 \\
$\mathrm{C} 54-\mathrm{H} 54 \cdots C g 1^{\mathrm{ii}}$ & 0.93 & 2.91 & $3.714(12)$ & 146 \\
$\mathrm{C} 553-\mathrm{H} 553 \cdots C g 1^{\mathrm{iii}}$ & 0.93 & 2.92 & $3.76(5)$ & 151 \\
\hline
\end{tabular}

Symmetry codes: (i) $x, y-1, z$; (ii) $-x+2,-y,-z+1$; (iii) $x-1, y, z$.

4-(4-Bromophenyl)-2-[5'-(2,4-dichlorophenoxy)-3'-methyl-1'-phenyl-5-(thiophen-2-yl)-3,4-dihydro-1' H,2H-3,4'bipyrazole-2-yl]-4-thiazole (III)

\section{Crystal data}

$\mathrm{C}_{32} \mathrm{H}_{22} \mathrm{BrCl}_{2} \mathrm{~N}_{5} \mathrm{OS}_{2}$

$M_{r}=707.47$

Triclinic, $P \overline{1}$

$a=12.3200(9) \AA$

$b=12.5700$ (9) $\AA$

$c=12.7742(9) \AA$

$\alpha=117.202(8)^{\circ}$

$\beta=102.879(7)^{\circ}$

$\gamma=105.727(7)^{\circ}$

$V=1548.4(2) \AA^{3}$

\section{Data collection}

Oxford Diffraction Xcalibur with Sapphire CCD detector diffractometer

Radiation source: Enhance (Mo) X-ray Source Graphite monochromator $\omega$ scans
$Z=2$

$F(000)=716$

$D_{\mathrm{x}}=1.517 \mathrm{Mg} \mathrm{m}^{-3}$

Mo $K \alpha$ radiation, $\lambda=0.71073 \AA$

Cell parameters from 6664 reflections

$\theta=2.6-27.8^{\circ}$

$\mu=1.67 \mathrm{~mm}^{-1}$

$T=296 \mathrm{~K}$

Plate, yellow

$0.40 \times 0.40 \times 0.08 \mathrm{~mm}$

Absorption correction: multi-scan

(CrysAlis RED; Oxford Diffraction, 2009)

$T_{\min }=0.779, T_{\max }=0.875$

10549 measured reflections

5773 independent reflections

3158 reflections with $I>2 \sigma(I)$ 


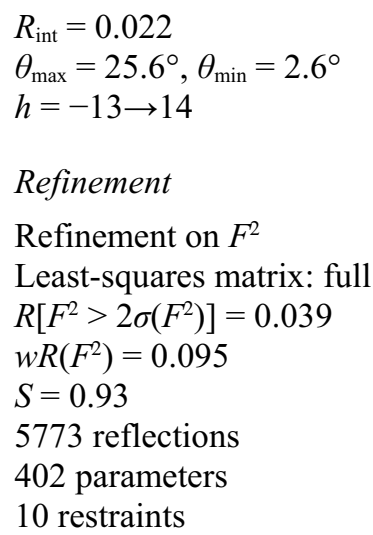

$R_{\text {int }}=0.022$

$\theta_{\max }=25.6^{\circ}, \theta_{\mathrm{min}}=2.6^{\circ}$

Refinement

Refinement on $F^{2}$

Least-squares matrix: full

$R\left[F^{2}>2 \sigma\left(F^{2}\right)\right]=0.039$

$S=0.93$

5773 reflections

10 restraints

$k=-15 \rightarrow 10$
$l=-10 \rightarrow 15$

Primary atom site location: difference Fourier map

Hydrogen site location: inferred from neighbouring sites

$\mathrm{H}$-atom parameters constrained

$w=1 /\left[\sigma^{2}\left(F_{\mathrm{o}}{ }^{2}\right)+(0.0475 P)^{2}\right]$

where $P=\left(F_{\mathrm{o}}^{2}+2 F_{\mathrm{c}}^{2}\right) / 3$

$(\Delta / \sigma)_{\max }<0.001$

$\Delta \rho_{\max }=0.47 \mathrm{e} \AA^{-3}$

$\Delta \rho_{\min }=-0.34$ e $\AA^{-3}$

Special details

Experimental. CrysAlis RED, Oxford Diffraction Ltd., 2009 Empirical absorption correction using spherical harmonics, implemented in SCALE3 ABSPACK scaling algorithm.

Geometry. All esds (except the esd in the dihedral angle between two 1.s. planes) are estimated using the full covariance matrix. The cell esds are taken into account individually in the estimation of esds in distances, angles and torsion angles; correlations between esds in cell parameters are only used when they are defined by crystal symmetry. An approximate (isotropic) treatment of cell esds is used for estimating esds involving l.s. planes.

Fractional atomic coordinates and isotropic or equivalent isotropic displacement parameters $\left(\AA^{2}\right)$

\begin{tabular}{|c|c|c|c|c|c|}
\hline & $x$ & $y$ & $z$ & $U_{\text {iso }} * / U_{\text {eq }}$ & Occ. $(<1)$ \\
\hline N1 & $0.5457(2)$ & $0.3735(2)$ & $0.86216(19)$ & $0.0478(5)$ & \\
\hline N2 & $0.4327(2)$ & $0.3754(2)$ & $0.8277(2)$ & $0.0514(6)$ & \\
\hline $\mathrm{C} 3$ & 0.4368 & $0.4319(2)$ & $0.7616(2)$ & $0.0495(7)$ & \\
\hline $\mathrm{C} 4$ & 0.5508 & $0.4674(2)$ & $0.7522(2)$ & $0.0477(7)$ & \\
\hline $\mathrm{C} 5$ & $0.6162(2)$ & $0.4278(2)$ & $0.8157(2)$ & $0.0446(6)$ & \\
\hline $\mathrm{C} 11$ & 0.5693 & $0.3119(2)$ & $0.9294(2)$ & $0.0449(6)$ & \\
\hline $\mathrm{C} 12$ & $0.6877(3)$ & $0.3383(3)$ & $0.9963(3)$ & $0.0552(7)$ & \\
\hline H12 & 0.7546 & 0.3982 & 0.9997 & $0.066^{*}$ & \\
\hline $\mathrm{C} 13$ & $0.7064(3)$ & $0.2750(3)$ & $1.0586(3)$ & $0.0591(8)$ & \\
\hline H13 & 0.7863 & 0.2915 & 1.1029 & $0.071^{*}$ & \\
\hline $\mathrm{C} 14$ & 0.6089 & $0.1887(3)$ & $1.0558(3)$ & $0.0630(8)$ & \\
\hline H14 & 0.6221 & 0.1465 & 1.0978 & $0.076^{*}$ & \\
\hline $\mathrm{C} 15$ & 0.4917 (3) & $0.1647(3)$ & 0.9907 (3) & $0.0719(9)$ & \\
\hline H15 & 0.4252 & 0.1064 & 0.9893 & $0.086^{*}$ & \\
\hline $\mathrm{C} 16$ & $0.4707(3)$ & $0.2257(3)$ & $0.9271(3)$ & $0.0617(8)$ & \\
\hline H16 & 0.3906 & 0.2088 & 0.8830 & $0.074^{*}$ & \\
\hline $\mathrm{C} 31$ & $0.3270(3)$ & $0.4476(3)$ & $0.7047(3)$ & $0.0686(8)$ & \\
\hline H31A & 0.3053 & 0.4075 & 0.6140 & $0.103 *$ & \\
\hline H31B & 0.3464 & 0.5402 & 0.7453 & $0.103^{*}$ & \\
\hline $\mathrm{H} 31 \mathrm{C}$ & 0.2589 & 0.4054 & 0.7181 & $0.103 *$ & \\
\hline N41 & 0.7375 & $0.5162(3)$ & $0.5905(3)$ & $0.0661(7)$ & \\
\hline N42 & $0.6211(2)$ & $0.4547(3)$ & $0.5794(2)$ & $0.0670(7)$ & \\
\hline $\mathrm{C} 43$ & $0.5928(3)$ & $0.5358(3)$ & $0.6881(3)$ & $0.0576(7)$ & \\
\hline $\mathrm{H} 43$ & 0.5288 & 0.5591 & 0.6567 & $0.069^{*}$ & \\
\hline
\end{tabular}




\begin{tabular}{|c|c|c|c|c|c|}
\hline $\mathrm{C} 44$ & 0.7168 & 0.6608 & 0.7729 & $0.0672(8)$ & \\
\hline $\mathrm{H} 44 \mathrm{~A}$ & 0.7058 & 0.7390 & 0.7866 & $0.081^{*}$ & \\
\hline H44B & 0.7546 & 0.6741 & 0.8558 & $0.081^{*}$ & \\
\hline $\mathrm{C} 45$ & $0.7931(3)$ & 0.6315 & 0.6965 & $0.0622(8)$ & \\
\hline S421 & $0.59658(8)$ & $0.25102(9)$ & $0.35914(8)$ & $0.0776(3)$ & \\
\hline $\mathrm{C} 422$ & $0.5442(3)$ & $0.3311(3)$ & $0.4739(3)$ & $0.0576(8)$ & \\
\hline N423 & $0.4313(2)$ & $0.2671(2)$ & $0.4522(2)$ & $0.0554(6)$ & \\
\hline C424 & 0.3781 & $0.1417(3)$ & $0.3379(3)$ & $0.0568(7)$ & \\
\hline $\mathrm{C} 425$ & $0.4545(3)$ & $0.1182(3)$ & 0.2773 & $0.0724(9)$ & \\
\hline H425 & 0.4325 & 0.0394 & 0.2004 & $0.087 *$ & \\
\hline $\mathrm{C} 441$ & $0.2509(3)$ & 0.0523 & 0.2964 & $0.0557(7)$ & \\
\hline $\mathrm{C} 442$ & $0.1828(3)$ & $0.0877(3)$ & 0.3669 & $0.0663(8)$ & \\
\hline H442 & 0.2188 & 0.1712 & 0.4429 & $0.080 *$ & \\
\hline C443 & $0.0635(3)$ & $0.0037(3)$ & $0.3287(3)$ & $0.0738(9)$ & \\
\hline H443 & 0.0196 & 0.0310 & 0.3783 & $0.089^{*}$ & \\
\hline C444 & 0.0085 & -0.1208 & $0.2172(3)$ & $0.0667(8)$ & \\
\hline $\mathrm{Br} 44$ & $-0.15403(3)$ & -0.23859 (4) & $0.16704(4)$ & 0.09399 (17) & \\
\hline C445 & $0.0740(4)$ & -0.1594 (3) & 0.1453 & $0.0803(10)$ & \\
\hline H445 & 0.0378 & -0.2435 & 0.0700 & $0.096^{*}$ & \\
\hline C446 & $0.1919(4)$ & $-0.0751(3)$ & $0.1836(3)$ & $0.0778(9)$ & \\
\hline H446 & 0.2349 & -0.1030 & 0.1331 & $0.093^{*}$ & \\
\hline S451 & $0.99714(13)$ & $0.68706(13)$ & $0.64070(13)$ & $0.1055(6)$ & $0.947(4)$ \\
\hline $\mathrm{C} 452$ & $0.9177(3)$ & $0.7199(4)$ & $0.7353(4)$ & $0.0739(9)$ & $0.947(4)$ \\
\hline C453 & $0.9844(6)$ & $0.8384(6)$ & $0.8487(6)$ & $0.0934(17)$ & $0.947(4)$ \\
\hline H453 & 0.9561 & 0.8733 & 0.9127 & $0.112^{*}$ & $0.947(4)$ \\
\hline $\mathrm{C} 454$ & $1.1069(5)$ & $0.9043(5)$ & $0.8577(6)$ & $0.1124(18)$ & $0.947(4)$ \\
\hline H454 & 1.1665 & 0.9865 & 0.9289 & $0.135^{*}$ & $0.947(4)$ \\
\hline $\mathrm{C} 455$ & $1.1232(5)$ & $0.8339(6)$ & $0.7532(7)$ & 0.1145 (18) & $0.947(4)$ \\
\hline H455 & 1.1955 & 0.8612 & 0.7421 & $0.137 *$ & $0.947(4)$ \\
\hline S551 & $1.025(3)$ & $0.871(2)$ & $0.863(3)$ & 0.0934 (17) & $0.053(4)$ \\
\hline C552 & $0.9177(3)$ & $0.7199(4)$ & $0.7353(4)$ & $0.0739(9)$ & $0.053(4)$ \\
\hline C553 & $0.966(5)$ & $0.658(5)$ & $0.654(6)$ & $0.1055(6)$ & $0.053(4)$ \\
\hline H553 & 0.9264 & 0.5696 & 0.5857 & $0.127^{*}$ & $0.053(4)$ \\
\hline C554 & $1.090(5)$ & $0.750(6)$ & $0.687(7)$ & 0.1145 (18) & $0.053(4)$ \\
\hline H554 & 1.1374 & 0.7270 & 0.6403 & $0.137^{*}$ & $0.053(4)$ \\
\hline C555 & $1.126(7)$ & $0.868(8)$ & $0.790(10)$ & $0.1124(18)$ & $0.053(4)$ \\
\hline H555 & 1.1972 & 0.9419 & 0.8192 & $0.135^{*}$ & $0.053(4)$ \\
\hline O51 & $0.73172(16)$ & $0.43438(16)$ & $0.83109(15)$ & $0.0493(4)$ & \\
\hline C51 & $0.7387(2)$ & $0.3304(2)$ & $0.7303(2)$ & $0.0440(6)$ & \\
\hline C52 & $0.8521(2)$ & $0.3502(3)$ & $0.7240(3)$ & $0.0488(7)$ & \\
\hline $\mathrm{Cl} 152$ & $0.97805(7)$ & $0.50033(8)$ & $0.83960(9)$ & $0.0895(3)$ & \\
\hline C53 & $0.8662(3)$ & $0.2526(3)$ & $0.6269(3)$ & $0.0586(8)$ & \\
\hline H53 & 0.9429 & 0.2664 & 0.6230 & $0.070^{*}$ & \\
\hline C54 & 0.7648 & $0.1344(3)$ & $0.5356(3)$ & $0.0563(7)$ & \\
\hline $\mathrm{Cl54}$ & $0.77954(9)$ & $0.01019(9)$ & $0.41070(8)$ & $0.0873(3)$ & \\
\hline $\mathrm{C} 55$ & $0.6533(3)$ & $0.1136(3)$ & $0.5414(3)$ & $0.0634(8)$ & \\
\hline H55 & 0.5856 & 0.0330 & 0.4793 & $0.076^{*}$ & \\
\hline C56 & $0.6397(3)$ & 0.2115 & $0.6391(3)$ & $0.0576(7)$ & \\
\hline
\end{tabular}


Atomic displacement parameters $\left(\AA^{2}\right)$

\begin{tabular}{|c|c|c|c|c|c|c|}
\hline & $U^{11}$ & $U^{22}$ & $U^{33}$ & $U^{12}$ & $U^{13}$ & $U^{23}$ \\
\hline N1 & $0.0546(15)$ & $0.0491(13)$ & $0.0455(13)$ & $0.0245(12)$ & $0.0228(11)$ & $0.0279(12)$ \\
\hline N2 & $0.0558(15)$ & $0.0592(14)$ & $0.0494(14)$ & $0.0323(12)$ & $0.0271(12)$ & $0.0304(12)$ \\
\hline $\mathrm{C} 3$ & $0.0627(19)$ & $0.0521(17)$ & $0.0406(16)$ & $0.0336(15)$ & $0.0240(14)$ & $0.0241(14)$ \\
\hline $\mathrm{C} 4$ & $0.0656(19)$ & $0.0472(16)$ & $0.0404(16)$ & $0.0306(15)$ & $0.0266(14)$ & $0.0257(14)$ \\
\hline $\mathrm{C} 5$ & $0.0547(18)$ & $0.0403(15)$ & $0.0406(15)$ & $0.0226(14)$ & $0.0252(14)$ & $0.0198(13)$ \\
\hline $\mathrm{C} 11$ & $0.0557(18)$ & $0.0434(15)$ & $0.0406(15)$ & $0.0250(14)$ & $0.0224(14)$ & $0.0235(13)$ \\
\hline $\mathrm{C} 12$ & $0.062(2)$ & $0.0595(18)$ & $0.0545(17)$ & $0.0272(16)$ & $0.0278(15)$ & $0.0363(16)$ \\
\hline $\mathrm{C} 13$ & $0.065(2)$ & $0.070(2)$ & 0.0525 & $0.0350(18)$ & $0.0262(16)$ & $0.0367(17)$ \\
\hline $\mathrm{C} 14$ & $0.090(2)$ & $0.060(2)$ & $0.0539(19)$ & $0.0401(19)$ & $0.0315(18)$ & $0.0366(16)$ \\
\hline $\mathrm{C} 15$ & $0.079(2)$ & $0.068(2)$ & $0.080(2)$ & $0.0250(19)$ & $0.0351(19)$ & $0.0506(19)$ \\
\hline $\mathrm{C} 16$ & $0.0580(18)$ & $0.066(2)$ & $0.067(2)$ & $0.0224(17)$ & $0.0225(16)$ & $0.0438(18)$ \\
\hline $\mathrm{C} 31$ & $0.085(2)$ & $0.083(2)$ & $0.065(2)$ & $0.054(2)$ & $0.0393(18)$ & $0.0460(18)$ \\
\hline N41 & $0.080(2)$ & 0.0741 (19) & $0.0642(18)$ & $0.0358(17)$ & $0.0382(16)$ & $0.0471(17)$ \\
\hline N42 & $0.0780(19)$ & $0.0714(18)$ & $0.0550(17)$ & $0.0272(16)$ & $0.0397(15)$ & $0.0343(15)$ \\
\hline $\mathrm{C} 43$ & $0.074(2)$ & 0.0604 (19) & $0.0558(18)$ & $0.0365(18)$ & $0.0330(17)$ & $0.0377(16)$ \\
\hline $\mathrm{C} 44$ & $0.089(2)$ & 0.0558 (19) & $0.066(2)$ & 0.0305 (19) & $0.0310(19)$ & 0.0409 (17) \\
\hline $\mathrm{C} 45$ & 0.078 & $0.071(2)$ & $0.068(2)$ & 0.039 & $0.036(2)$ & $0.054(2)$ \\
\hline S421 & $0.0958(7)$ & $0.0867(6)$ & $0.0679(5)$ & $0.0459(6)$ & $0.0536(5)$ & $0.0425(5)$ \\
\hline $\mathrm{C} 422$ & $0.080(2)$ & $0.067(2)$ & $0.0479(19)$ & $0.0399(19)$ & $0.0355(17)$ & $0.0392(18)$ \\
\hline N423 & $0.0729(18)$ & $0.0614(16)$ & $0.0449(14)$ & $0.0360(15)$ & $0.0317(13)$ & $0.0311(13)$ \\
\hline $\mathrm{C} 424$ & $0.080(2)$ & $0.062(2)$ & $0.0451(18)$ & $0.0416(19)$ & $0.0316(17)$ & $0.0329(17)$ \\
\hline $\mathrm{C} 425$ & $0.095(2)$ & $0.072(2)$ & $0.059(2)$ & $0.044(2)$ & 0.0440 (19) & $0.0326(17)$ \\
\hline $\mathrm{C} 441$ & $0.081(2)$ & 0.0597 (19) & $0.0423(17)$ & $0.0442(18)$ & $0.0298(16)$ & $0.0295(15)$ \\
\hline $\mathrm{C} 442$ & $0.078(2)$ & $0.060(2)$ & $0.0572(19)$ & $0.041(2)$ & $0.0311(18)$ & $0.0224(16)$ \\
\hline $\mathrm{C} 443$ & $0.077(2)$ & $0.070(2)$ & $0.070(2)$ & $0.042(2)$ & 0.0344 (19) & 0.0274 (19) \\
\hline $\mathrm{C} 444$ & $0.076(2)$ & $0.069(2)$ & $0.068(2)$ & $0.0457(19)$ & $0.0303(19)$ & $0.0385(19)$ \\
\hline $\mathrm{Br} 44$ & $0.0795(3)$ & $0.0793(3)$ & $0.1006(3)$ & $0.0331(2)$ & $0.0273(2)$ & $0.0381(2)$ \\
\hline $\mathrm{C} 445$ & $0.092(3)$ & $0.061(2)$ & $0.064(2)$ & $0.031(2)$ & $0.031(2)$ & $0.0186(18)$ \\
\hline C446 & $0.101(3)$ & $0.078(3)$ & $0.061(2)$ & $0.048(2)$ & $0.048(2)$ & $0.031(2)$ \\
\hline S451 & $0.0958(12)$ & $0.1464(12)$ & $0.1214(10)$ & $0.0581(10)$ & $0.0620(8)$ & $0.0961(9)$ \\
\hline $\mathrm{C} 452$ & $0.074(2)$ & $0.078(2)$ & 0.088 & $0.030(2)$ & $0.028(2)$ & $0.062(2)$ \\
\hline $\mathrm{C} 453$ & 0.067 (4) & $0.080(4)$ & $0.124(4)$ & $0.012(3)$ & $0.028(3)$ & $0.065(3)$ \\
\hline $\mathrm{C} 454$ & $0.076(4)$ & $0.112(4)$ & $0.155(5)$ & $0.026(3)$ & $0.034(3)$ & $0.091(4)$ \\
\hline $\mathrm{C} 455$ & $0.075(3)$ & $0.149(5)$ & $0.171(6)$ & $0.044(4)$ & $0.046(4)$ & $0.128(5)$ \\
\hline S551 & $0.067(4)$ & $0.080(4)$ & $0.124(4)$ & $0.012(3)$ & 0.028 & $0.065(3)$ \\
\hline C552 & $0.074(2)$ & $0.078(2)$ & 0.088 & $0.030(2)$ & $0.028(2)$ & $0.062(2)$ \\
\hline C553 & $0.0958(12)$ & $0.1464(12)$ & $0.1214(10)$ & $0.0581(10)$ & $0.0620(8)$ & $0.0961(9)$ \\
\hline C554 & 0.075 & $0.149(5)$ & $0.171(6)$ & $0.044(4)$ & $0.046(4)$ & $0.128(5)$ \\
\hline C555 & $0.076(4)$ & $0.112(4)$ & $0.155(5)$ & $0.026(3)$ & $0.034(3)$ & $0.091(4)$ \\
\hline O51 & $0.0512(12)$ & $0.0460(11)$ & $0.0451(11)$ & $0.0196(9)$ & $0.0208(9)$ & $0.0212(9)$ \\
\hline $\mathrm{C} 51$ & $0.0533(18)$ & $0.0436(16)$ & $0.0432(16)$ & $0.0229(15)$ & $0.0241(14)$ & $0.0266(14)$ \\
\hline C52 & $0.0421(16)$ & $0.0517(17)$ & $0.0502(17)$ & $0.0159(14)$ & $0.0166(14)$ & $0.0299(15)$ \\
\hline $\mathrm{Cl} 152$ & $0.0503(5)$ & $0.0766(6)$ & $0.0886(6)$ & 0.0101 & $0.0132(4)$ & $0.0238(5)$ \\
\hline
\end{tabular}


supporting information

\begin{tabular}{lllllll} 
C53 & $0.0549(19)$ & $0.077(2)$ & $0.064(2)$ & $0.0381(18)$ & $0.0339(17)$ & $0.0431(19)$ \\
C54 & $0.073(2)$ & $0.062(2)$ & $0.0535(18)$ & $0.0405(18)$ & $0.0372(17)$ & $0.0341(17)$ \\
C154 & $0.1139(7)$ & $0.0907(6)$ & $0.0691(5)$ & $0.0620(6)$ & $0.0543(5)$ & $0.0350(5)$ \\
C55 & $0.063(2)$ & $0.0437(17)$ & $0.064(2)$ & $0.0156(15)$ & $0.0306(16)$ & $0.0180(15)$ \\
C56 & $0.0493(17)$ & $0.0473(18)$ & $0.068(2)$ & $0.0167(15)$ & $0.0322(16)$ & $0.0242(16)$ \\
\hline
\end{tabular}

Geometric parameters $\left(A,{ }^{\circ}\right)$

\begin{tabular}{|c|c|c|c|}
\hline $\mathrm{N} 1-\mathrm{C} 5$ & $1.361(3)$ & $\mathrm{C} 425-\mathrm{H} 425$ & 0.9300 \\
\hline $\mathrm{N} 1-\mathrm{N} 2$ & $1.374(3)$ & $\mathrm{C} 441-\mathrm{C} 442$ & $1.373(4)$ \\
\hline $\mathrm{N} 1-\mathrm{C} 11$ & $1.428(3)$ & $\mathrm{C} 441-\mathrm{C} 446$ & $1.400(4)$ \\
\hline $\mathrm{N} 2-\mathrm{C} 3$ & $1.330(3)$ & $\mathrm{C} 442-\mathrm{C} 443$ & $1.372(4)$ \\
\hline $\mathrm{C} 3-\mathrm{C} 4$ & $1.404(3)$ & $\mathrm{C} 442-\mathrm{H} 442$ & 0.9300 \\
\hline $\mathrm{C} 3-\mathrm{C} 31$ & 1.495 & $\mathrm{C} 443-\mathrm{C} 444$ & $1.377(4)$ \\
\hline $\mathrm{C} 4-\mathrm{C} 5$ & $1.361(3)$ & $\mathrm{C} 443-\mathrm{H} 443$ & 0.9300 \\
\hline $\mathrm{C} 4-\mathrm{C} 43$ & $1.506(3)$ & $\mathrm{C} 444-\mathrm{C} 445$ & $1.366(4)$ \\
\hline $\mathrm{C} 5-\mathrm{O} 51$ & $1.367(3)$ & $\mathrm{C} 444-\mathrm{Br} 44$ & $1.888(3)$ \\
\hline $\mathrm{C} 11-\mathrm{C} 12$ & 1.375 & $\mathrm{C} 445-\mathrm{C} 446$ & $1.359(4)$ \\
\hline $\mathrm{C} 11-\mathrm{C} 16$ & $1.375(3)$ & $\mathrm{C} 445-\mathrm{H} 445$ & 0.9300 \\
\hline $\mathrm{C} 12-\mathrm{C} 13$ & $1.385(3)$ & $\mathrm{C} 446-\mathrm{H} 446$ & 0.9300 \\
\hline $\mathrm{C} 12-\mathrm{H} 12$ & 0.9300 & $\mathrm{~S} 451-\mathrm{C} 452$ & $1.696(4)$ \\
\hline $\mathrm{C} 13-\mathrm{C} 14$ & $1.364(4)$ & $\mathrm{S} 451-\mathrm{C} 455$ & $1.706(6)$ \\
\hline $\mathrm{C} 13-\mathrm{H} 13$ & 0.9300 & $\mathrm{C} 452-\mathrm{C} 453$ & $1.351(7)$ \\
\hline $\mathrm{C} 14-\mathrm{C} 15$ & $1.366(4)$ & $\mathrm{C} 453-\mathrm{C} 454$ & $1.461(10)$ \\
\hline $\mathrm{C} 14-\mathrm{H} 14$ & 0.9300 & $\mathrm{C} 453-\mathrm{H} 453$ & 0.9300 \\
\hline $\mathrm{C} 15-\mathrm{C} 16$ & $1.379(4)$ & $\mathrm{C} 454-\mathrm{C} 455$ & $1.321(7)$ \\
\hline $\mathrm{C} 15-\mathrm{H} 15$ & 0.9300 & $\mathrm{C} 454-\mathrm{H} 454$ & 0.9300 \\
\hline $\mathrm{C} 16-\mathrm{H} 16$ & 0.9300 & $\mathrm{C} 455-\mathrm{H} 455$ & 0.9300 \\
\hline C31-H31A & 0.9600 & S551-C555 & $1.707(12)$ \\
\hline $\mathrm{C} 31-\mathrm{H} 31 \mathrm{~B}$ & 0.9600 & C553-C554 & $1.461(14)$ \\
\hline C31-H31C & 0.9600 & С553-H553 & 0.9300 \\
\hline $\mathrm{N} 41-\mathrm{C} 45$ & $1.287(4)$ & C554-C555 & $1.322(12)$ \\
\hline $\mathrm{N} 41-\mathrm{N} 42$ & $1.372(3)$ & С554-H554 & 0.9300 \\
\hline $\mathrm{N} 42-\mathrm{C} 422$ & $1.359(4)$ & C555-H555 & 0.9300 \\
\hline $\mathrm{N} 42-\mathrm{C} 43$ & $1.484(3)$ & $\mathrm{O} 51-\mathrm{C} 51$ & $1.395(3)$ \\
\hline $\mathrm{C} 43-\mathrm{C} 44$ & $1.543(4)$ & $\mathrm{C} 51-\mathrm{C} 56$ & $1.366(3)$ \\
\hline $\mathrm{C} 43-\mathrm{H} 43$ & 0.9800 & $\mathrm{C} 51-\mathrm{C} 52$ & $1.379(3)$ \\
\hline $\mathrm{C} 44-\mathrm{C} 45$ & $1.496(4)$ & $\mathrm{C} 52-\mathrm{C} 53$ & $1.377(4)$ \\
\hline $\mathrm{C} 44-\mathrm{H} 44 \mathrm{~A}$ & 0.9700 & $\mathrm{C} 52-\mathrm{Cl} 52$ & $1.730(3)$ \\
\hline $\mathrm{C} 44-\mathrm{H} 44 \mathrm{~B}$ & 0.9700 & $\mathrm{C} 53-\mathrm{C} 54$ & $1.374(4)$ \\
\hline $\mathrm{C} 45-\mathrm{C} 452$ & $1.439(4)$ & $\mathrm{C} 53-\mathrm{H} 53$ & 0.9300 \\
\hline $\mathrm{S} 421-\mathrm{C} 425$ & $1.717(3)$ & $\mathrm{C} 54-\mathrm{C} 55$ & $1.353(4)$ \\
\hline $\mathrm{S} 421-\mathrm{C} 422$ & $1.734(3)$ & $\mathrm{C} 54-\mathrm{Cl} 54$ & $1.737(3)$ \\
\hline $\mathrm{C} 422-\mathrm{N} 423$ & $1.295(3)$ & $\mathrm{C} 55-\mathrm{C} 56$ & $1.379(4)$ \\
\hline $\mathrm{N} 423-\mathrm{C} 424$ & $1.399(3)$ & $\mathrm{C} 55-\mathrm{H} 55$ & 0.9300 \\
\hline $\mathrm{C} 424-\mathrm{C} 425$ & $1.356(4)$ & C56-H56 & 0.9300 \\
\hline C424-C441 & $1.460(4)$ & & \\
\hline
\end{tabular}




\begin{tabular}{|c|c|c|c|}
\hline $\mathrm{C} 5-\mathrm{N} 1-\mathrm{N} 2$ & $109.84(19)$ & $\mathrm{C} 425-\mathrm{C} 424-\mathrm{C} 441$ & $127.3(3)$ \\
\hline $\mathrm{C} 5-\mathrm{N} 1-\mathrm{C} 11$ & $130.5(2)$ & $\mathrm{N} 423-\mathrm{C} 424-\mathrm{C} 441$ & $118.8(3)$ \\
\hline $\mathrm{N} 2-\mathrm{N} 1-\mathrm{C} 11$ & $119.5(2)$ & $\mathrm{C} 424-\mathrm{C} 425-\mathrm{S} 421$ & $112.0(2)$ \\
\hline $\mathrm{C} 3-\mathrm{N} 2-\mathrm{N} 1$ & $105.3(2)$ & $\mathrm{C} 424-\mathrm{C} 425-\mathrm{H} 425$ & 124.0 \\
\hline $\mathrm{N} 2-\mathrm{C} 3-\mathrm{C} 4$ & $111.7(2)$ & $\mathrm{S} 421-\mathrm{C} 425-\mathrm{H} 425$ & 124.0 \\
\hline $\mathrm{N} 2-\mathrm{C} 3-\mathrm{C} 31$ & $120.5(2)$ & $\mathrm{C} 442-\mathrm{C} 441-\mathrm{C} 446$ & $116.2(3)$ \\
\hline $\mathrm{C} 4-\mathrm{C} 3-\mathrm{C} 31$ & $127.7(2)$ & $\mathrm{C} 442-\mathrm{C} 441-\mathrm{C} 424$ & $121.8(3)$ \\
\hline $\mathrm{C} 5-\mathrm{C} 4-\mathrm{C} 3$ & $104.5(2)$ & $\mathrm{C} 446-\mathrm{C} 441-\mathrm{C} 424$ & $122.0(3)$ \\
\hline $\mathrm{C} 5-\mathrm{C} 4-\mathrm{C} 43$ & $127.4(3)$ & $\mathrm{C} 443-\mathrm{C} 442-\mathrm{C} 441$ & $122.0(3)$ \\
\hline $\mathrm{C} 3-\mathrm{C} 4-\mathrm{C} 43$ & $128.1(2)$ & $\mathrm{C} 443-\mathrm{C} 442-\mathrm{H} 442$ & 119.0 \\
\hline $\mathrm{C} 4-\mathrm{C} 5-\mathrm{N} 1$ & $108.7(2)$ & $\mathrm{C} 441-\mathrm{C} 442-\mathrm{H} 442$ & 119.0 \\
\hline $\mathrm{C} 4-\mathrm{C} 5-\mathrm{O} 51$ & $128.6(2)$ & $\mathrm{C} 442-\mathrm{C} 443-\mathrm{C} 444$ & $120.1(3)$ \\
\hline $\mathrm{N} 1-\mathrm{C} 5-\mathrm{O} 51$ & $122.7(2)$ & $\mathrm{C} 442-\mathrm{C} 443-\mathrm{H} 443$ & 119.9 \\
\hline $\mathrm{C} 12-\mathrm{C} 11-\mathrm{C} 16$ & $120.1(2)$ & $\mathrm{C} 444-\mathrm{C} 443-\mathrm{H} 443$ & 119.9 \\
\hline $\mathrm{C} 12-\mathrm{C} 11-\mathrm{N} 1$ & $121.3(2)$ & $\mathrm{C} 445-\mathrm{C} 444-\mathrm{C} 443$ & $119.3(3)$ \\
\hline $\mathrm{C} 16-\mathrm{C} 11-\mathrm{N} 1$ & $118.6(2)$ & $\mathrm{C} 445-\mathrm{C} 444-\mathrm{Br} 44$ & $120.4(3)$ \\
\hline $\mathrm{C} 11-\mathrm{C} 12-\mathrm{C} 13$ & $119.4(3)$ & $\mathrm{C} 443-\mathrm{C} 444-\mathrm{Br} 44$ & $120.3(3)$ \\
\hline $\mathrm{C} 11-\mathrm{C} 12-\mathrm{H} 12$ & 120.3 & $\mathrm{C} 446-\mathrm{C} 445-\mathrm{C} 444$ & $120.1(3)$ \\
\hline $\mathrm{C} 13-\mathrm{C} 12-\mathrm{H} 12$ & 120.3 & $\mathrm{C} 446-\mathrm{C} 445-\mathrm{H} 445$ & 120.0 \\
\hline $\mathrm{C} 14-\mathrm{C} 13-\mathrm{C} 12$ & $120.7(3)$ & $\mathrm{C} 444-\mathrm{C} 445-\mathrm{H} 445$ & 120.0 \\
\hline $\mathrm{C} 14-\mathrm{C} 13-\mathrm{H} 13$ & 119.7 & $\mathrm{C} 445-\mathrm{C} 446-\mathrm{C} 441$ & $122.3(3)$ \\
\hline $\mathrm{C} 12-\mathrm{C} 13-\mathrm{H} 13$ & 119.7 & $\mathrm{C} 445-\mathrm{C} 446-\mathrm{H} 446$ & 118.9 \\
\hline $\mathrm{C} 13-\mathrm{C} 14-\mathrm{C} 15$ & $119.5(3)$ & $\mathrm{C} 441-\mathrm{C} 446-\mathrm{H} 446$ & 118.9 \\
\hline $\mathrm{C} 13-\mathrm{C} 14-\mathrm{H} 14$ & 120.2 & $\mathrm{C} 452-\mathrm{S} 451-\mathrm{C} 455$ & $92.0(3)$ \\
\hline $\mathrm{C} 15-\mathrm{C} 14-\mathrm{H} 14$ & 120.2 & $\mathrm{C} 453-\mathrm{C} 452-\mathrm{C} 45$ & $124.7(4)$ \\
\hline $\mathrm{C} 14-\mathrm{C} 15-\mathrm{C} 16$ & $120.9(3)$ & $\mathrm{C} 453-\mathrm{C} 452-\mathrm{S} 451$ & $112.1(3)$ \\
\hline $\mathrm{C} 14-\mathrm{C} 15-\mathrm{H} 15$ & 119.6 & $\mathrm{C} 45-\mathrm{C} 452-\mathrm{S} 451$ & $123.2(3)$ \\
\hline $\mathrm{C} 16-\mathrm{C} 15-\mathrm{H} 15$ & 119.6 & $\mathrm{C} 452-\mathrm{C} 453-\mathrm{C} 454$ & $111.1(6)$ \\
\hline $\mathrm{C} 11-\mathrm{C} 16-\mathrm{C} 15$ & $119.4(3)$ & $\mathrm{C} 452-\mathrm{C} 453-\mathrm{H} 453$ & 124.4 \\
\hline $\mathrm{C} 11-\mathrm{C} 16-\mathrm{H} 16$ & 120.3 & $\mathrm{C} 454-\mathrm{C} 453-\mathrm{H} 453$ & 124.4 \\
\hline $\mathrm{C} 15-\mathrm{C} 16-\mathrm{H} 16$ & 120.3 & $\mathrm{C} 455-\mathrm{C} 454-\mathrm{C} 453$ & $112.2(5)$ \\
\hline $\mathrm{C} 3-\mathrm{C} 31-\mathrm{H} 31 \mathrm{~A}$ & 109.5 & $\mathrm{C} 455-\mathrm{C} 454-\mathrm{H} 454$ & 123.9 \\
\hline $\mathrm{C} 3-\mathrm{C} 31-\mathrm{H} 31 \mathrm{~B}$ & 109.5 & $\mathrm{C} 453-\mathrm{C} 454-\mathrm{H} 454$ & 123.9 \\
\hline $\mathrm{H} 31 \mathrm{~A}-\mathrm{C} 31-\mathrm{H} 31 \mathrm{~B}$ & 109.5 & $\mathrm{C} 454-\mathrm{C} 455-\mathrm{S} 451$ & $112.5(4)$ \\
\hline $\mathrm{C} 3-\mathrm{C} 31-\mathrm{H} 31 \mathrm{C}$ & 109.5 & $\mathrm{C} 454-\mathrm{C} 455-\mathrm{H} 455$ & 123.8 \\
\hline $\mathrm{H} 31 \mathrm{~A}-\mathrm{C} 31-\mathrm{H} 31 \mathrm{C}$ & 109.5 & $\mathrm{~S} 451-\mathrm{C} 455-\mathrm{H} 455$ & 123.8 \\
\hline $\mathrm{H} 31 \mathrm{~B}-\mathrm{C} 31-\mathrm{H} 31 \mathrm{C}$ & 109.5 & $\mathrm{C} 554-\mathrm{C} 553-\mathrm{H} 553$ & 124.5 \\
\hline $\mathrm{C} 45-\mathrm{N} 41-\mathrm{N} 42$ & $108.5(3)$ & $\mathrm{C} 555-\mathrm{C} 554-\mathrm{C} 553$ & $111.9(12)$ \\
\hline $\mathrm{C} 422-\mathrm{N} 42-\mathrm{N} 41$ & $119.3(3)$ & С555-C554-H554 & 124.0 \\
\hline $\mathrm{C} 422-\mathrm{N} 42-\mathrm{C} 43$ & $126.7(3)$ & C553-C554-H554 & 124.0 \\
\hline $\mathrm{N} 41-\mathrm{N} 42-\mathrm{C} 43$ & $114.0(2)$ & C554-C555-S551 & $112.0(15)$ \\
\hline $\mathrm{N} 42-\mathrm{C} 43-\mathrm{C} 4$ & $113.5(2)$ & $\mathrm{C} 554-\mathrm{C} 555-\mathrm{H} 555$ & 124.0 \\
\hline $\mathrm{N} 42-\mathrm{C} 43-\mathrm{C} 44$ & $100.3(2)$ & S551-C555-H555 & 124.0 \\
\hline $\mathrm{C} 4-\mathrm{C} 43-\mathrm{C} 44$ & $115.3(2)$ & $\mathrm{C} 5-\mathrm{O} 51-\mathrm{C} 51$ & $115.95(19)$ \\
\hline $\mathrm{N} 42-\mathrm{C} 43-\mathrm{H} 43$ & 109.1 & $\mathrm{C} 56-\mathrm{C} 51-\mathrm{C} 52$ & $119.1(2)$ \\
\hline $\mathrm{C} 4-\mathrm{C} 43-\mathrm{H} 43$ & 109.1 & $\mathrm{C} 56-\mathrm{C} 51-\mathrm{O} 51$ & $123.4(2)$ \\
\hline $\mathrm{C} 44-\mathrm{C} 43-\mathrm{H} 43$ & 109.1 & $\mathrm{C} 52-\mathrm{C} 51-\mathrm{O} 51$ & $117.5(2)$ \\
\hline $\mathrm{C} 45-\mathrm{C} 44-\mathrm{C} 43$ & $103.5(2)$ & $\mathrm{C} 53-\mathrm{C} 52-\mathrm{C} 51$ & 120.9 \\
\hline
\end{tabular}




\begin{tabular}{|c|c|c|c|}
\hline $\mathrm{C} 45-\mathrm{C} 44-\mathrm{H} 44 \mathrm{~A}$ & 111.1 & $\mathrm{C} 53-\mathrm{C} 52-\mathrm{Cl} 52$ & $119.7(2)$ \\
\hline $\mathrm{C} 43-\mathrm{C} 44-\mathrm{H} 44 \mathrm{~A}$ & 111.1 & $\mathrm{C} 51-\mathrm{C} 52-\mathrm{Cl} 52$ & $119.4(2)$ \\
\hline $\mathrm{C} 45-\mathrm{C} 44-\mathrm{H} 44 \mathrm{~B}$ & 111.1 & $\mathrm{C} 54-\mathrm{C} 53-\mathrm{C} 52$ & $118.8(3)$ \\
\hline $\mathrm{C} 43-\mathrm{C} 44-\mathrm{H} 44 \mathrm{~B}$ & 111.1 & $\mathrm{C} 54-\mathrm{C} 53-\mathrm{H} 53$ & 120.6 \\
\hline $\mathrm{H} 44 \mathrm{~A}-\mathrm{C} 44-\mathrm{H} 44 \mathrm{~B}$ & 109.0 & $\mathrm{C} 52-\mathrm{C} 53-\mathrm{H} 53$ & 120.6 \\
\hline $\mathrm{N} 41-\mathrm{C} 45-\mathrm{C} 452$ & $121.5(3)$ & $\mathrm{C} 55-\mathrm{C} 54-\mathrm{C} 53$ & $120.7(3)$ \\
\hline $\mathrm{N} 41-\mathrm{C} 45-\mathrm{C} 44$ & $113.6(3)$ & $\mathrm{C} 55-\mathrm{C} 54-\mathrm{Cl} 54$ & $119.5(2)$ \\
\hline $\mathrm{C} 452-\mathrm{C} 45-\mathrm{C} 44$ & $124.8(3)$ & $\mathrm{C} 53-\mathrm{C} 54-\mathrm{Cl} 54$ & $119.8(2)$ \\
\hline $\mathrm{C} 425-\mathrm{S} 421-\mathrm{C} 422$ & $87.62(16)$ & $\mathrm{C} 54-\mathrm{C} 55-\mathrm{C} 56$ & $120.3(3)$ \\
\hline $\mathrm{N} 423-\mathrm{C} 422-\mathrm{N} 42$ & $123.9(3)$ & $\mathrm{C} 54-\mathrm{C} 55-\mathrm{H} 55$ & 119.8 \\
\hline $\mathrm{N} 423-\mathrm{C} 422-\mathrm{S} 421$ & $116.5(2)$ & $\mathrm{C} 56-\mathrm{C} 55-\mathrm{H} 55$ & 119.8 \\
\hline $\mathrm{N} 42-\mathrm{C} 422-\mathrm{S} 421$ & $119.6(3)$ & $\mathrm{C} 51-\mathrm{C} 56-\mathrm{C} 55$ & $120.1(3)$ \\
\hline $\mathrm{C} 422-\mathrm{N} 423-\mathrm{C} 424$ & $109.9(3)$ & $\mathrm{C} 51-\mathrm{C} 56-\mathrm{H} 56$ & 119.9 \\
\hline $\mathrm{C} 425-\mathrm{C} 424-\mathrm{N} 423$ & $113.9(3)$ & $\mathrm{C} 55-\mathrm{C} 56-\mathrm{H} 56$ & 119.9 \\
\hline $\mathrm{C} 5-\mathrm{N} 1-\mathrm{N} 2-\mathrm{C} 3$ & $-0.4(3)$ & $\mathrm{N} 42-\mathrm{C} 422-\mathrm{N} 423-\mathrm{C} 424$ & $-178.4(2)$ \\
\hline $\mathrm{C} 11-\mathrm{N} 1-\mathrm{N} 2-\mathrm{C} 3$ & $-176.7(2)$ & $\mathrm{S} 421-\mathrm{C} 422-\mathrm{N} 423-\mathrm{C} 424$ & $1.8(3)$ \\
\hline $\mathrm{N} 1-\mathrm{N} 2-\mathrm{C} 3-\mathrm{C} 4$ & $-0.1(3)$ & $\mathrm{C} 422-\mathrm{N} 423-\mathrm{C} 424-\mathrm{C} 425$ & $-1.0(3)$ \\
\hline $\mathrm{N} 1-\mathrm{N} 2-\mathrm{C} 3-\mathrm{C} 31$ & $178.2(2)$ & $\mathrm{C} 422-\mathrm{N} 423-\mathrm{C} 424-\mathrm{C} 441$ & $179.2(2)$ \\
\hline $\mathrm{N} 2-\mathrm{C} 3-\mathrm{C} 4-\mathrm{C} 5$ & $0.6(3)$ & $\mathrm{N} 423-\mathrm{C} 424-\mathrm{C} 425-\mathrm{S} 421$ & $-0.1(3)$ \\
\hline $\mathrm{C} 31-\mathrm{C} 3-\mathrm{C} 4-\mathrm{C} 5$ & $-177.6(2)$ & $\mathrm{C} 441-\mathrm{C} 424-\mathrm{C} 425-\mathrm{S} 421$ & $179.6(2)$ \\
\hline $\mathrm{N} 2-\mathrm{C} 3-\mathrm{C} 4-\mathrm{C} 43$ & $-178.6(2)$ & $\mathrm{C} 422-\mathrm{S} 421-\mathrm{C} 425-\mathrm{C} 424$ & $0.9(2)$ \\
\hline $\mathrm{C} 31-\mathrm{C} 3-\mathrm{C} 4-\mathrm{C} 43$ & $3.2(4)$ & $\mathrm{C} 425-\mathrm{C} 424-\mathrm{C} 441-\mathrm{C} 442$ & $179.8(3)$ \\
\hline $\mathrm{C} 3-\mathrm{C} 4-\mathrm{C} 5-\mathrm{N} 1$ & $-0.8(3)$ & $\mathrm{N} 423-\mathrm{C} 424-\mathrm{C} 441-\mathrm{C} 442$ & $-0.5(4)$ \\
\hline $\mathrm{C} 43-\mathrm{C} 4-\mathrm{C} 5-\mathrm{N} 1$ & $178.3(2)$ & $\mathrm{C} 425-\mathrm{C} 424-\mathrm{C} 441-\mathrm{C} 446$ & $0.8(4)$ \\
\hline $\mathrm{C} 3-\mathrm{C} 4-\mathrm{C} 5-\mathrm{O} 51$ & $177.3(2)$ & $\mathrm{N} 423-\mathrm{C} 424-\mathrm{C} 441-\mathrm{C} 446$ & $-179.5(2)$ \\
\hline $\mathrm{C} 43-\mathrm{C} 4-\mathrm{C} 5-\mathrm{O} 51$ & $-3.5(4)$ & $\mathrm{C} 446-\mathrm{C} 441-\mathrm{C} 442-\mathrm{C} 443$ & $-0.5(4)$ \\
\hline $\mathrm{N} 2-\mathrm{N} 1-\mathrm{C} 5-\mathrm{C} 4$ & $0.8(3)$ & $\mathrm{C} 424-\mathrm{C} 441-\mathrm{C} 442-\mathrm{C} 443$ & $-179.6(3)$ \\
\hline $\mathrm{C} 11-\mathrm{N} 1-\mathrm{C} 5-\mathrm{C} 4$ & $176.5(2)$ & $\mathrm{C} 441-\mathrm{C} 442-\mathrm{C} 443-\mathrm{C} 444$ & $0.5(5)$ \\
\hline $\mathrm{N} 2-\mathrm{N} 1-\mathrm{C} 5-\mathrm{O} 51$ & $-177.4(2)$ & $\mathrm{C} 442-\mathrm{C} 443-\mathrm{C} 444-\mathrm{C} 445$ & $-0.1(5)$ \\
\hline $\mathrm{C} 11-\mathrm{N} 1-\mathrm{C} 5-\mathrm{O} 51$ & $-1.7(4)$ & $\mathrm{C} 442-\mathrm{C} 443-\mathrm{C} 444-\mathrm{Br} 44$ & $178.1(2)$ \\
\hline $\mathrm{C} 5-\mathrm{N} 1-\mathrm{C} 11-\mathrm{C} 12$ & $22.9(4)$ & $\mathrm{C} 443-\mathrm{C} 444-\mathrm{C} 445-\mathrm{C} 446$ & $-0.3(5)$ \\
\hline $\mathrm{N} 2-\mathrm{N} 1-\mathrm{C} 11-\mathrm{C} 12$ & $-161.7(2)$ & $\mathrm{Br} 44-\mathrm{C} 444-\mathrm{C} 445-\mathrm{C} 446$ & $-178.5(2)$ \\
\hline $\mathrm{C} 5-\mathrm{N} 1-\mathrm{C} 11-\mathrm{C} 16$ & $-157.7(3)$ & $\mathrm{C} 444-\mathrm{C} 445-\mathrm{C} 446-\mathrm{C} 441$ & $0.3(5)$ \\
\hline $\mathrm{N} 2-\mathrm{N} 1-\mathrm{C} 11-\mathrm{C} 16$ & $17.7(3)$ & $\mathrm{C} 442-\mathrm{C} 441-\mathrm{C} 446-\mathrm{C} 445$ & $0.1(4)$ \\
\hline $\mathrm{C} 16-\mathrm{C} 11-\mathrm{C} 12-\mathrm{C} 13$ & $1.5(4)$ & $\mathrm{C} 424-\mathrm{C} 441-\mathrm{C} 446-\mathrm{C} 445$ & $179.2(3)$ \\
\hline $\mathrm{N} 1-\mathrm{C} 11-\mathrm{C} 12-\mathrm{C} 13$ & $-179.1(2)$ & $\mathrm{N} 41-\mathrm{C} 45-\mathrm{C} 452-\mathrm{C} 453$ & $-175.4(5)$ \\
\hline $\mathrm{C} 11-\mathrm{C} 12-\mathrm{C} 13-\mathrm{C} 14$ & $-1.0(4)$ & $\mathrm{C} 44-\mathrm{C} 45-\mathrm{C} 452-\mathrm{C} 453$ & $3.4(6)$ \\
\hline $\mathrm{C} 12-\mathrm{C} 13-\mathrm{C} 14-\mathrm{C} 15$ & $0.0(4)$ & $\mathrm{N} 41-\mathrm{C} 45-\mathrm{C} 452-\mathrm{S} 451$ & $5.6(4)$ \\
\hline $\mathrm{C} 13-\mathrm{C} 14-\mathrm{C} 15-\mathrm{C} 16$ & $0.5(5)$ & $\mathrm{C} 44-\mathrm{C} 45-\mathrm{C} 452-\mathrm{S} 451$ & $-175.5(2)$ \\
\hline $\mathrm{C} 12-\mathrm{C} 11-\mathrm{C} 16-\mathrm{C} 15$ & $-1.0(4)$ & $\mathrm{C} 455-\mathrm{S} 451-\mathrm{C} 452-\mathrm{C} 453$ & $-0.1(4)$ \\
\hline $\mathrm{N} 1-\mathrm{C} 11-\mathrm{C} 16-\mathrm{C} 15$ & $179.6(2)$ & $\mathrm{C} 455-\mathrm{S} 451-\mathrm{C} 452-\mathrm{C} 45$ & $179.0(3)$ \\
\hline $\mathrm{C} 14-\mathrm{C} 15-\mathrm{C} 16-\mathrm{C} 11$ & $0.0(4)$ & $\mathrm{C} 45-\mathrm{C} 452-\mathrm{C} 453-\mathrm{C} 454$ & $-179.4(4)$ \\
\hline $\mathrm{C} 45-\mathrm{N} 41-\mathrm{N} 42-\mathrm{C} 422$ & $179.6(2)$ & $\mathrm{S} 451-\mathrm{C} 452-\mathrm{C} 453-\mathrm{C} 454$ & $-0.3(6)$ \\
\hline $\mathrm{C} 45-\mathrm{N} 41-\mathrm{N} 42-\mathrm{C} 43$ & $0.5(3)$ & $\mathrm{C} 452-\mathrm{C} 453-\mathrm{C} 454-\mathrm{C} 455$ & $0.6(7)$ \\
\hline $\mathrm{C} 422-\mathrm{N} 42-\mathrm{C} 43-\mathrm{C} 4$ & $55.9(4)$ & $\mathrm{C} 453-\mathrm{C} 454-\mathrm{C} 455-\mathrm{S} 451$ & $-0.6(7)$ \\
\hline $\mathrm{N} 41-\mathrm{N} 42-\mathrm{C} 43-\mathrm{C} 4$ & $-125.0(3)$ & $\mathrm{C} 452-\mathrm{S} 451-\mathrm{C} 455-\mathrm{C} 454$ & $0.4(5)$ \\
\hline $\mathrm{C} 422-\mathrm{N} 42-\mathrm{C} 43-\mathrm{C} 44$ & $179.5(2)$ & C553-C554-C555-S551 & $8(16)$ \\
\hline
\end{tabular}




$\begin{array}{ll}\mathrm{N} 41-\mathrm{N} 42-\mathrm{C} 43-\mathrm{C} 44 & -1.4(3) \\ \mathrm{C} 5-\mathrm{C} 4-\mathrm{C} 43-\mathrm{N} 42 & 63.0(4) \\ \mathrm{C} 3-\mathrm{C} 4-\mathrm{C} 43-\mathrm{N} 42 & -118.0(3) \\ \mathrm{C} 5-\mathrm{C} 4-\mathrm{C} 43-\mathrm{C} 44 & -51.9(4) \\ \mathrm{C} 3-\mathrm{C} 4-\mathrm{C} 43-\mathrm{C} 44 & 127.1(3) \\ \mathrm{N} 42-\mathrm{C} 43-\mathrm{C} 44-\mathrm{C} 45 & 1.7(2) \\ \mathrm{C} 4-\mathrm{C} 43-\mathrm{C} 44-\mathrm{C} 45 & 124.0(2) \\ \mathrm{N} 42-\mathrm{N} 41-\mathrm{C} 45-\mathrm{C} 452 & 179.8(2) \\ \mathrm{N} 42-\mathrm{N} 41-\mathrm{C} 45-\mathrm{C} 44 & 0.8(3) \\ \mathrm{C} 43-\mathrm{C} 44-\mathrm{C} 45-\mathrm{N} 41 & -1.7(3) \\ \mathrm{C} 43-\mathrm{C} 44-\mathrm{C} 45-\mathrm{C} 452 & 179.3(2) \\ \mathrm{N} 41-\mathrm{N} 42-\mathrm{C} 422-\mathrm{N} 423 & -177.2(2) \\ \mathrm{C} 43-\mathrm{N} 42-\mathrm{C} 422-\mathrm{N} 423 & 1.9(4) \\ \mathrm{N} 41-\mathrm{N} 42-\mathrm{C} 422-\mathrm{S} 421 & 2.6(3) \\ \mathrm{C} 43-\mathrm{N} 42-\mathrm{C} 422-\mathrm{S} 421 & -178.31(19) \\ \mathrm{C} 425-\mathrm{S} 421-\mathrm{C} 422-\mathrm{N} 423 & -1.6(2) \\ \mathrm{C} 425-\mathrm{S} 421-\mathrm{C} 422-\mathrm{N} 42 & 178.6(2) \\ \end{array}$

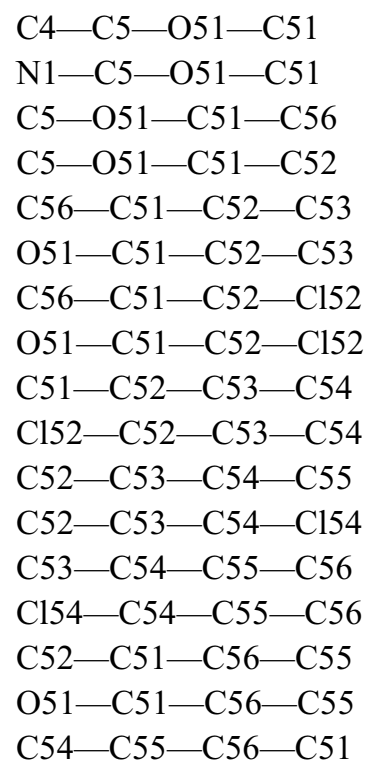

$-84.1(3)$

$93.9(3)$

$-17.9(3)$

$162.1(2)$

$0.5(4)$

$-179.5(2)$

$-179.80(19)$

$0.2(3)$

$0.1(4)$

$-179.63(19)$

-0.5 (4)

$179.2(2)$

$0.3(4)$

$-179.3(2)$

-0.7 (4)

$179.4(2)$

$0.3(4)$ 